\title{
THE ONE-DIMENSIONAL STOCHASTIC KELLER-SEGEL MODEL WITH TIME-HOMOGENEOUS SPATIAL WIENER PROCESSES
}

\author{
ERIKA HAUSENBLAS, DEBOPRIYA MUKHERJEE, AND THANH TRAN
}

\begin{abstract}
Chemotaxis is a fundamental mechanism of cells and organisms, which is responsible for attracting microbes to food, embryonic cells into developing tissues, or immune cells to infection sites. Mathematically chemotaxis is described by the Patlak-Keller-Segel model. This macroscopic system of equations is derived from the microscopic model when limiting behaviour is studied. However, on taking the limit and passing from the microscopic equations to the macroscopic equations, fluctuations are neglected. Perturbing the system by a Gaussian random field restitutes the inherent randomness of the system. This gives us the motivation to study the classical Patlak-Keller-Segel system perturbed by random processes.

We study a stochastic version of the classical Patlak-Keller-Segel system under homogeneous Neumann boundary conditions on an interval $\mathcal{O}=[0,1]$. In particular, let $\mathcal{W}_{1}, \mathcal{W}_{2}$ be two timehomogeneous spatial Wiener processes over a filtered probability space $\mathfrak{A}$. Let $u$ and $v$ denote the cell density and concentration of the chemical signal. We investigate the coupled system

$$
\left\{\begin{array}{r}
d u-\left(r_{u} \Delta u-\chi \operatorname{div}(u \nabla v)\right) d t=u \circ d \mathcal{W}_{1}, \\
d v-\left(r_{v} \Delta v-\alpha v\right) d t=\beta u d t+v \circ d \mathcal{W}_{2},
\end{array}\right.
$$

with initial conditions $(u(0), v(0))=\left(u_{0}, v_{0}\right)$. The positive terms $r_{u}$ and $r_{v}$ are the diffusivity of the cells and chemoattractant, respectively, the positive value $\chi$ is the chemotactic sensitivity, $\alpha \geq 0$ is the so-called damping constant. The noise is interpreted in the Stratonovich sense. Given $T>0$, we will prove the existence of a martingale solution on $[0, T]$.
\end{abstract}

Keywords and phrases: Chemotaxis, Keller-Segel model, Stochastic Partial Differential Equations, Stochastic Analysis, Mathematical Biology

AMS subject classification (2002): Primary 60H15, 92C17, 35A01; Secondary 35B65, 35K87, 35K51, 35Q92.

\section{INTRODUCTION}

Chemotaxis is defined as the oriented movement of cells (or an organism) in response to a chemical gradient. Many sorts of motile cells undergo chemotaxis. For example, bacteria and many amoeboid cells can move in the direction of a food source. In our bodies, immune cells like macrophages and neutrophils can move towards invading cells. Other cells, connected with the immune response and wound healing, are attracted to areas of inflammation by chemical signals.

Theoretical and mathematical modeling of chemotaxis dates back to the works of Patlak [26] in the 1950s and Keller and Segel 22 in the 1970s. Let $A=\Delta$ be the Laplace operator with

Date: September 30, 2020.

The first two authors of the paper are supported by Austrian Science Foundation, project number P 32295. The first author is also supported by Science Visiting Research Fellowship, UNSW Sydney, during the time of her visit to UNSW. The last two authors are partially supported by ARC DP160101755. 
Neumann boundary conditions (see equation (2.1)). The simplest form of the model is

$$
\left\{\begin{array}{l}
\dot{u}(t)=r_{u} A u(t)-\chi \operatorname{div}(u(t) \nabla v(t)), \quad t \in \mathbb{R}_{0}^{+} \\
\dot{v}(t)=r_{v} A v(t)+\beta u(t)-\alpha v(t)
\end{array}\right.
$$

with initial conditions $(u(0, x), v(0, x))=\left(u_{0}(x), v_{0}(x)\right)$, where $x \in \mathcal{O}=[0,1]$. Here, $u$ denotes the cell density, and $v$ is the concentration of the chemical signal. The positive terms $r_{u}$ and $r_{v}$ are the diffusivity of the cells and chemoattractant, respectively. The positive constant $\chi$ is the chemotactic sensitivity. In the signal concentration model, $\alpha \geq 0$ is the so-called damping constant. For more details we refer to the surveys by Horstmann [19, 20], Hillen and Painter [18, and Bellomo et. al. 3], and the work of Biler [5], or Perthame [28].

This macroscopic system of equations is derived from the limiting behaviour of the microscopic model; see, e.g. [30]. Here, one considers the density of the population modelled by Fick's laws, in which the reactions are viewed as functions of the density of cells and the concentration of the chemoattractant. From the microscopic perspective, one interprets the movement of species populations as a result of microscopic irregular movement of single members of the considered population. In the derivation of the above macroscopic equations, fluctuations around the mean value are neglected. For a more realistic model, it is necessary to consider essential features of the natural environment which are non-reproducible. Hence, the model should include random spatio-temporal forcing.

Another source of randomness is due to the environment or the fluctuation of parameters. Here, the constant $\alpha$ or $\chi$ may fluctuate randomly in time. Biologists (see [27] or [17]) distinguish between internal (or intrinsic) noise caused by the irregular movement of the cells and external noise caused by a random environment. An appropriate mathematical approach to establish more realistic models is the incorporation of randomness in the system.

In this article, we perturb the density of cells $u$ and concentration of chemoattractant $v$ by time-homogeneous spatial Wiener processes, i.e. an infinite dimensional Wiener process depending on the time and space variables (For a brief discussion on different approaches to infinite dimensional Wiener processes we refer to [10]). The perturbation is multiplicative, and the integral is interpreted in the Stratonovich sense. As our main result, we show the existence of a martingale solution. We want to emphasise here that there are several obstacles to overcome in the stochastic case, compared to the deterministic one. Due to the nonlinearity in the system, one has to truncate nonlinear terms and use stopping time arguments. Here, one can only rely on the entities which are controlled by a Lyapunov functional. This essentially means that in the proof we are not able to work in spaces with high integrability or high regularity. Hence, we are unable to show the necessary estimates which are required by the Banach Fixed Point Theorem. Thus we construct an integral operator, and use compactness and a stochastic version of the Schauder-Tychonoff Fixed Point Theorem to obtain a solution. More precisely, we prove a stochastic version of the Schauder-Tychonoff Fixed Point Theorem which is specific to our problem. In this manner, we achieve only the existence of a martingale solution, but not the uniqueness of the solution.

The authors are not aware of any work which treats the simple Keller-Segel model with noise. We can only find the very recent work [36], where the deterministic Keller-Segel model is coupled with the stochastic NavierStokes equations. 


\section{The Main Result}

Let $\mathcal{H}_{1}$ and $\mathcal{H}_{2}$ be two Hilbert spaces and let $T>0$; later on in Assumption 2.3 we will see that $\mathcal{H}_{1}$ and $\mathcal{H}_{2}$ are some Bessel potential spaces. Let $\mathfrak{A}=(\Omega, \mathcal{F}, \mathbb{F}, \mathbb{P})$ be a complete probability space and $\mathbb{F}=\left(\mathcal{F}_{t}\right)_{t \in[0, T]}$ be a filtration satisfying the usual conditions i.e.,

(i) $\mathbb{P}$ is complete on $(\Omega, \mathcal{F})$,

(ii) for each $t \geq 0, \mathcal{F}_{t}$ contains all $(\mathcal{F}, \mathbb{P})$-null sets and

(iii) the filtration $\left(\mathcal{F}_{t}\right)_{t \in[0, T]}$ is right-continuous.

Let $\mathcal{W}_{1}$ and $\mathcal{W}_{2}$ be two cylindrical Wiener processes defined over $\mathfrak{A}$ on Hilbert spaces $\mathcal{H}_{1}$ and $\mathcal{H}_{2}$, respectively. (This means these Wiener processes are defined using orthonormal bases of $\mathcal{H}_{1}$ and $\mathcal{H}_{2}$.) Let us define the Laplacian with the Neumann boundary conditions by

$$
\begin{cases}D(A) & :=\left\{u \in H^{2}(0,1): u_{x}(0)=u_{x}(1)=0\right\}, \\ A u & :=\Delta u=u_{x x}, \quad u \in D(A),\end{cases}
$$

and let us recall that the positive terms $r_{u}$ and $r_{v}$ are the diffusivity of the cells and chemoattractant, respectively. We consider the following system of equations in $\mathcal{O}=[0,1]$

$$
\left\{\begin{array}{c}
d u-\left(r_{u} A u-\chi \operatorname{div}(u \nabla v)\right) d t=u \circ d \mathcal{W}_{1} \\
d v-\left(r_{v} A v-\alpha v\right) d t=\beta u d t+v \circ d \mathcal{W}_{2}
\end{array}\right.
$$

with the initial conditions given by $(u(0), v(0))=\left(u_{0}, v_{0}\right)$. The first equation of system (2.2) is nonlinear in $u$ and $v$, and the second equation is linear in $u$ and $v$.

Since we model an intrinsic noise, the stochastic integral above is interpreted in the Stratonovich sense. Since the white noise is an approximation of a continuously fluctuating noise with finite memory being much shorter than the dynamical timescales, the representation of the stochastic integral as a Stratonovich stochastic integral is appropriate. For a detailed explanation of the Stratonovich integral, we refer to the book by Duan and Wang [13] or to the original work of Stratonovich [31]. For completeness we add the definition of strong solutions to system (2.2). For a Banach space $E$, the space $C_{b}^{0}([0, T] ; E)$ is the set of all continuous and bounded functions $u:[0, T] \rightarrow E$.

Definition 2.1. We call a pair of processes $(u, v)$ a strong solution to system (2.2) if $u$ : $[0, T] \times \Omega \rightarrow L^{2}(\mathcal{O})$ and $v:[0, T] \times \Omega \rightarrow H^{1}(\mathcal{O})$ are $\mathbb{F}$-progressively measurable processe $\mathbb{1}$ such that $\mathbb{P}$-a.s. $(u, v) \in C_{b}^{0}\left([0, T] ; L^{2}(\mathcal{O})\right) \times C_{b}^{0}\left([0, T] ; H^{1}(\mathcal{O})\right)$ and satisfy for all $t \in[0, T]$ and $\mathbb{P}$-a.s. the integral equation

$$
\begin{aligned}
& u(t)=e^{t r_{u} A} u_{0}-\chi \int_{0}^{t} e^{(t-s) r_{u} A} \operatorname{div}(u(s) \nabla v(s)) d s+\int_{0}^{t} e^{(t-s) r_{u} A} u(s) \circ d \mathcal{W}_{1}(s), \\
& v(t)=e^{t\left(r_{v} A-\alpha I\right)} v_{0}+\beta \int_{0}^{t} e^{(t-s)\left(r_{v} A-\alpha I\right)} u(s) d s+\int_{0}^{t} e^{(t-s)\left(r_{v} A-\alpha I\right)} v(s) \circ d \mathcal{W}_{2}(s) .
\end{aligned}
$$

Since we use compactness methods to show the existence of the solution, the given probability space will be lost, and we have to construct another probability space. In this way, we will obtain a martingale solution, which is also known as a weak solution in the probabilistic sense.

\footnotetext{
${ }^{1}$ The process $\xi:[0, T] \times \Omega \rightarrow \mathbb{X}$ is said to be progressively measurable over a probability space $\left(\Omega, \mathcal{F},\left(\mathcal{F}_{t}\right)_{t \in[0, T]}, \mathbb{P}\right)$ if, for every time $t \in[0, T]$, the map $[0, t] \times \Omega \rightarrow \mathbb{X}$ defined by $(s, \omega) \mapsto \xi_{s}(\omega)$ is $\operatorname{Borel}([0, t]) \otimes \mathcal{F}_{t^{-}}$ measurable. This implies that $\xi$ is $\left(\mathcal{F}_{t}\right)_{t \in[0, T] \text {-adapted. }}$
} 
Definition 2.2. A martingale solution to the problem (2.2) is a tuple $\left(\mathfrak{A},\left(\mathcal{W}_{1}, \mathcal{W}_{2}\right),(u, v)\right)$ such that

(i) $\mathfrak{A}=(\Omega, \mathcal{F}, \mathbb{F}, \mathbb{P})$ is a complete filtered probability space with a filtration $\mathbb{F}=\left(\mathcal{F}_{t}\right)_{t \in[0, T]}$ satisfying the usual conditions,

(ii) $\left(\mathcal{W}_{1}, \mathcal{W}_{2}\right)$ is a pair of cylindrical Wiener processes over $\mathfrak{A}$ on some given Hilbert space $\mathcal{H}_{1} \times \mathcal{H}_{2}$

(iii) $u:[0, T] \times \Omega \rightarrow L^{2}(\mathcal{O})$ and $v:[0, T] \times \Omega \rightarrow H^{1}(\mathcal{O})$ are two $\mathbb{F}$-progressively measurable processes being a strong solution to (2.2) over $\mathfrak{A}$.

Let us consider the complete orthonormal system of the underlying Lebesgue space $L^{2}(\mathcal{O})$ given by the trigonometric functions (see [14, p. 352])

$$
\psi_{k}(x)= \begin{cases}\sqrt{2} \sin (2 \pi k x) & \text { if } k \geq 1, x \in \mathcal{O}, \\ 1 & \text { if } k=0, x \in \mathcal{O}, \\ \sqrt{2} \cos (2 \pi k x) & \text { if } k \leq-1, x \in \mathcal{O}\end{cases}
$$

For the proof of the existence of the solution, the Wiener perturbation needs to satisfy regularity assumptions given in the next hypotheses.

Assumption 2.3. Let $\mathcal{H}_{1}$ and $\mathcal{H}_{2}$ be two Bessel potential spaces such that the embeddings $\iota_{1}: \mathcal{H}_{1} \hookrightarrow L^{2}(\mathcal{O})$ and $\iota_{2}: \mathcal{H}_{2} \hookrightarrow H^{1}(\mathcal{O})$ are Hilbert-Schmidt operators. The Wiener processes $\mathcal{W}_{1}$ and $\mathcal{W}_{2}$ are two cylindrical processes on $\mathcal{H}_{1}$ and $\mathcal{H}_{2}$.

Remark 2.4. As an example we can take $\mathcal{H}_{1}=H^{\delta_{1}}(\mathcal{O})$ for $\delta_{1}>1$, and $\mathcal{H}_{2}=H^{\delta_{2}}(\mathcal{O})$ for $\delta_{2}>2$ where $H^{\delta_{i}}(\mathcal{O}), i=1,2$, is to be defined in Notation 2.1. The Wiener processes $\mathcal{W}_{1}$ and $\mathcal{W}_{2}$ are then given by

$$
\mathcal{W}_{1}(t, x)=\sum_{k \in \mathbb{Z}} \psi_{k}^{\left(\delta_{1}\right)}(x) \beta_{k}^{(1)}(t) \quad \text { and } \quad \mathcal{W}_{2}(t, x)=\sum_{k \in \mathbb{Z}} \psi_{k}^{\left(\delta_{2}\right)}(x) \beta_{k}^{(2)}(t)
$$

where $\psi_{k}^{\left(\delta_{i}\right)}:=\left(1+(2 \pi k)^{2}\right)^{-\delta_{i} / 2} \psi_{k}, i=1,2$, with $\psi_{k}$ defined by (2.3), and where $\left\{\beta_{k}^{(i)}: k \in\right.$ $\mathbb{Z}\}, i=1,2$, are two families of mutually independent identical distributed standard Brownian motions. We note that $\left\{\psi_{k}^{\left(\delta_{i}\right)}: k \in \mathbb{Z}\right\}$ forms an orthonormal system in $H^{\delta_{i}}(\mathcal{O}), i=1,2$. We will also write

$$
\mathcal{W}_{i}(t, x)=\sum_{k \in \mathbb{Z}} \lambda_{k}^{i} \psi_{k}(x) \beta_{k}^{(i)}(t), \quad i=1,2,
$$

where, for $i=1,2$, the sequence $\left\{\lambda_{k}^{i}: k \in \mathbb{Z}\right\}, \lambda_{k}^{i}:=\left(1+(2 \pi k)^{2}\right)^{-\delta_{i} / 2}$, is non-negative with $\lambda_{k}^{i}=$ $\lambda_{-k}^{i}$ for all $k \in \mathbb{Z}$. For the later course of analysis, for each $i=1,2$, we define $\gamma_{i}:=\sum_{k \in \mathbb{Z}}\left(\lambda_{k}^{i}\right)^{2}$.

Since the solution $(u, v)$ represents the cell density and concentration of the chemical signal, $u$ and $v$ are non-negative. This immediately implies that the initial conditions $u_{0}$ and $v_{0}$ are non-negative. Besides, one needs to impose some more regularity assumptions on $u_{0}$ and $v_{0}$.

Assumption 2.5. Let $u_{0} \in L^{2}(\mathcal{O})$ and $v_{0} \in H^{1}(\mathcal{O})$ be two random variables over $\mathfrak{A}$ such that

(a) $u_{0} \geq 0$ and $v_{0} \geq 0$;

(b) $\left(u_{0}, v_{0}\right)$ is $\mathcal{F}_{0}-$ measurable;

(c) $\mathbb{E}\left[\left|u_{0}\right|_{L^{2}}^{2}\right]<\infty$ and $\mathbb{E}\left[\left|\nabla v_{0}\right|_{L^{2}}^{2}\right]<\infty$. 
Notation 2.1. Let $A_{1}$ be the positive Laplace operator $-\Delta$ (as an operator defined on $L^{2}(\mathbb{R})$ ) restricted to functions defined on $\mathcal{O}$, namely,

$$
A_{1}:=-\Delta, \quad D\left(A_{1}\right):=H^{2}(\mathcal{O}) \cap H_{0}^{1}(\mathcal{O}),
$$

and let $\left(\psi_{j}, \rho_{j}\right)$ be the eigenfunctions and eigenvalues of $A_{1}$. The Bessel potential space $H^{\kappa}(\mathcal{O})$ is defined by

$$
H^{\kappa}(\mathcal{O})=\left\{u=\sum_{j} a_{j} \psi_{j} \in L^{2}(\mathcal{O}):\|u\|_{H^{\kappa}(\mathcal{O})}=\left(\sum_{j} a_{j}^{2} \rho_{j}^{2 \kappa}\right)^{1 / 2}<\infty .\right\} .
$$

Notation 2.2. Let us define by LlogL $(\mathcal{O})$ the Zygmund space (see [4, Definition 6.1, p. 243]) consisting of all Lebesgue-measurable functions $f: \mathcal{O} \rightarrow \mathbb{R}$ for which $\int_{\mathcal{O}}|f(x)| \log (|f(x)|)^{+} d x<$ $\infty$. This space is equipped with the norm

$$
|f|_{L \log L}:=\int_{0}^{1} f^{*}(t) \log \left(\frac{1}{t}\right) d t
$$

where $f^{*}$ is defined by

$$
f^{*}(t)=\inf \left\{\lambda: \delta_{f}(\lambda) \leq t\right\}, \quad t \geq 0 .
$$

Here $\delta_{f}(\lambda)$ is the Lebesgue measure of the set $\{x \in \mathcal{O}:|f(x)|>\lambda\}, \lambda \geq 0$. We note that $f \in \operatorname{Llog} L$ iff $\int_{\mathcal{O}}|f(x)| \log (2+|f(x)|) d x<\infty$ (see p. 252 of [4]). Note also that by Theorem 6.5 p. 247 in [4] we have that $\operatorname{Llog} L(\mathcal{O}) \hookrightarrow L^{1}(\mathcal{O})$.

We now present the main result of the paper.

Theorem 2.6. Let Assumption 2.3 be satisfied. Then for all initial conditions $\left(u_{0}, v_{0}\right) \in$ $L^{2}(\mathcal{O}) \times H^{1}(\mathcal{O})$ satisfying Assumption 2.5 and for all $T>0$, there exists a martingale solution $\left(\mathfrak{A},\left(\mathcal{W}_{1}, \mathcal{W}_{2}\right),(u, v)\right)$ to the system (2.2). Moreover, there exists a positive constant $C=$ $C\left(T, u_{0}, v_{0}\right)$ such that

$$
\mathbb{E}\left[\sup _{0 \leq s \leq T}|u(s)|_{L^{1}}\right]+\mathbb{E}\left[\sup _{0 \leq s \leq T}|\nabla v(s)|_{L^{2}}^{2}\right]+\mathbb{E}\left[\int_{0}^{T}|\nabla v(s)|_{H^{1}}^{2} d s\right] \leq C .
$$

If in addition $\mathbb{E}\left[\left|u_{0}\right|_{L l o g L}^{p}\right]<\infty$ and $\mathbb{E}\left[\left|\nabla v_{0}\right|_{L^{2}}^{2 p}\right]<\infty$ for $p \geq 1$, then there exists a constant $C=C\left(p, T,\left(u_{0}, v_{0}\right)\right)>0$ such that

$$
\mathbb{E}\left[\sup _{0 \leq s \leq T}|u(s)|_{L^{1}}^{p}\right]+\mathbb{E}\left[\sup _{0 \leq s \leq T}|\nabla v(s)|_{L^{2}}^{2 p}\right]+\mathbb{E}\left(\int_{0}^{T}|\nabla v(s)|_{H^{1}}^{2} d s\right)^{p} \leq C .
$$

The proof of this main result is presented in the next section. As a consequence of the theorem, we obtain the following corollary on regularity, the proof of which is moved to Appendix A,

Corollary 2.7. Let $p>4$ and let $\beta, \delta>0$ be such that $\beta+\delta<1 / 2-1 / p$ and $\varrho=2 / 3$. Let Assumption 2.3 be satified. Then, if $\left(u_{0}, v_{0}\right) \in L^{2}(\mathcal{O}) \times H^{1}(\mathcal{O})$ satisfies Assumption 2.5 along with $\mathbb{E}\left[\left|u_{0}\right|_{L \log L}^{p}\right]<\infty$ and $\mathbb{E}\left[\left|\nabla v_{0}\right|_{L^{2}}^{2 p}\right]<\infty$, then the martingale solution $(u, v)$ to the system (2.2) satisfies

$$
\mathbb{E}\left\|u-e^{(\cdot)\left(r_{u} A+\gamma I\right)} u_{0}\right\|_{C_{b}^{\beta}\left([0, T] ; H^{2 \delta}\right)}^{\varrho p}<\infty \quad \text { and } \quad \mathbb{E}\left\|\nabla v-e^{(\cdot)\left(r_{v} A-\alpha I\right)} \nabla v_{0}\right\|_{C_{b}^{\beta}\left([0, T] ; H^{2 \delta}\right)}^{p}<\infty .
$$


Since the proof of Theorem 2.6 is quite technical and sophisticated, in the next section we split it into five main steps. In Step I, we consider the truncated system and show the existence of solutions by using the Schauder-Tychonoff Type Fixed Point Theorem. In this way we obtain a family of solutions $\left\{\left(u_{n}, v_{n}\right): n \in \mathbb{N}\right\}$ to the truncated system depending on the truncation parameter $n$. In Step II, we show that the solution of the truncated system is non-negative, provided the initial conditions are. In Step III, we construct a new pair of processes on the whole interval $[0, T]$ such that they are a solution of the chemotaxis system up to a stopping time, and satisfy the heat equation (with lower order terms). Then using an appropriate Lyapunov functional, we derive in Step IV uniform estimates in the $L^{1}$-norm of the family of solutions. In the final step (Step V), we prove the existence of solutions $\mathbb{P}$-a.s to the original problem on the whole time interval $[0, T]$.

Due to the fact that we cannot use higher regularity or integrability as given by the Lyapunov functional, we can only prove existence. However, we assume that one can find local solutions being unique.

\section{Proof of Theorem 2.6}

3.1. Conversion between the Itô and Stratonovich form of SPDEs. Before starting with the actual proof, we first outline shortly the difference between the Stratonovich integral and the Itô integral. A real fluctuating forcing term has a finite amplitude and a finite-timescale. In this way, the so-called white noise is an idealisation of the delta-function-correlated noise, and, as mentioned already in the introduction, the white noise can be interpreted as an approximation of a continuously fluctuating noise with finite memory being much shorter than the dynamical timescales. Wong and Zakai [35] investigate the convergence of ordinary differential equations (ODEs) which, in particular, involve piecewise linear approximations of a one-dimensional Wiener process. They show that the solutions to these ODEs converge almost surely to a solution of a specific Stratonovitch stochastic differential equation (SDE) and not an Itô SDE. In particular, they show that the proper representation of the white noise as a stochastic integral in the Stratonovich sense is appropriate. However, a drawback of the Stratonovich stochastic integral is that one does not have the BurkholderDavisGundy inequality at their disposal. Hence we use the relation between the Stratonovich and Itô integral to change the integral into an Itô stochastic integral. Therefore, we present the relation between the two stochastic integrals shortly. For a short discussion from the viewpoint of physicists, we refer to [21] and 24.

Let $\mathfrak{A}=(\Omega, \mathcal{F}, \mathbb{F}, \mathbb{P})$ be a complete probability space and $\mathbb{F}=\left(\mathcal{F}_{t}\right)_{t \in[0, T]}$ be a filtration satisfying the usual conditions. Let $H$ be a Hilbert space and

$$
\begin{gathered}
M_{\mathfrak{A}}^{2}(0, T ; H):=\{\xi:[0, T] \times \Omega \rightarrow H \mid \xi \text { is progressively measurable over } \mathfrak{A} \\
\text { and } \left.\mathbb{E}\left[\sup _{0 \leq s \leq T}|\xi(s)|_{H}^{2}\right]<\infty\right\} .
\end{gathered}
$$

This space is equipped with the norm

$$
\|\xi\|_{M_{\mathfrak{A}}^{2}}:=\left(\mathbb{E}\left[\sup _{0 \leq s \leq T}|\xi(s)|_{H}^{2}\right]\right)^{1 / 2} .
$$


The cylindrical Wiener process $\mathcal{W}$ is time-homogeneous spatial Wiener processes (see Assumption 2.3 and Remark 2.4) on a given Hilbert space $\mathcal{H}$ which can be written as

$$
\mathcal{W}(t, x)=\sum_{k \in \mathbb{N}} \phi_{k}(x) \beta_{k}(t), \quad t \in[0, T],
$$

where $\left\{\phi_{k}: k \in \mathbb{Z}\right\}$ is an orthonormal basis in $\mathcal{H}$ and $\left\{\beta_{k}: k \in \mathbb{Z}\right\}$ is a sequence of independent standard Brownian motions. A stochastic integral can be interpreted in different ways, e.g., as a Stratonovich integral or as an Itô integral. For the time being, let us assume that $\mathcal{W}$ is a cylindrical Wiener process on $\mathcal{H}$, where, for simplicity, we assume that the embedding $\mathcal{H} \hookrightarrow L^{2}(\mathcal{O})$ is a Hilbert-Schmidt operator. Let $\pi=\left\{t_{0}^{\pi}=0<t_{1}^{\pi}<\ldots<t_{N^{\pi}}^{\pi}=T\right\}$ be a partition of $[0, T]$ and let $|\pi|=\max _{1 \leq k \leq N^{\pi}}\left(t_{k}^{\pi}-t_{k-1}^{\pi}\right)$. The Stratonovich integral of a process $\xi$ is defined by

$$
\int_{0}^{t} \xi(s) \circ d \mathcal{W}(s):=\lim _{|\pi| \rightarrow 0} \sum_{k=1}^{N^{\pi}} \xi_{k}\left(\mathcal{W}\left(t_{k}^{\pi} \wedge t\right)-\mathcal{W}\left(t_{k-1}^{\pi} \wedge t\right)\right)
$$

with $\xi_{k}=\xi\left(\left(t_{k}^{\pi} \wedge t+t_{k-1}^{\pi} \wedge t\right) / 2\right)$, while the Itô integral is defined by

$$
\int_{0}^{t} \xi(s) d \mathcal{W}(s):=\lim _{|\pi| \rightarrow 0} \sum_{k=1}^{N^{\pi}} \xi_{k}\left(\mathcal{W}\left(t_{k}^{\pi} \wedge t\right)-\mathcal{W}\left(t_{k-1}^{\pi} \wedge t\right)\right)
$$

with $\xi_{k}=\xi\left(t_{k-1}^{\pi} \wedge t\right)$.

Let us consider the following stochastic partial differential equation (SPDE) perturbed by a one-dimensional Wiener process, i.e. $\mathcal{H}=\mathbb{R}$. The integral is expressed in the sense of the Stratonovich integral, i.e.,

$$
d \xi(t)=A \xi(t) d t+\sigma(\xi(t)) \circ d \mathcal{W}(t), \quad \xi(0)=\xi_{0} .
$$

Here $A$ is an analytic operator on the Hilbert space $H:=L^{2}(\mathcal{O})$ and $\sigma: H \rightarrow L_{H S}(\mathcal{H}, H)$ where $L_{\mathrm{HS}}\left(H_{1}, H_{2}\right)$ denotes the space of linear operators from $H_{1}$ to $H_{2}$ equipped with the Hilbert-Schmidt norm. As mentioned in the introduction, the Stratonovich integral is the natural one for modelling fluctuations. However, the disadvantage is that the Stratonovich integral is not a martingale; hence, neither the stochastic Itô-isometry nor the BurkholderDavis-Gundy inequality is available. Nevertheless, one can reformulate (3.2) into an SPDE with an Itô differential, by adding a correction term. The reason for the correction term is that, in the definition of the Stratonovitch integral, the random variable $\xi_{k}=\xi\left(\left(t_{k-1}^{\pi}+t_{k}^{\pi}\right) / 2\right)$ and the increment $\mathcal{W}\left(t_{k}^{\pi}\right)-\mathcal{W}\left(t_{k-1}^{\pi}\right)$ are not independent. The random variable $\xi_{k}$ contains some information about the difference $\mathcal{W}\left(t_{k}^{\pi}\right)-\mathcal{W}\left(t_{k-1}^{\pi}\right)$ because $\xi$ is evaluated at the midpoint of the interval. In the case of the Itô integral one chooses $\xi_{k}=\xi\left(t_{k-1}^{\pi}\right)$, the value of $\xi$ at the left endpoint of the interval. This random variable $\xi_{k}$ is independent of $\mathcal{W}\left(t_{k}^{\pi}\right)-\mathcal{W}\left(t_{k-1}^{\pi}\right)$.

The correction term can be calculated explicitly (see [13, p. 65, Section 4.5.1]), i.e., the equivalent Itô equation of (3.2) is given by

$$
d \xi(t)=A \xi(t) d t+\frac{1}{2} D_{\xi} \sigma(\xi(t)) \sigma(\xi(t)) d t+\sigma(\xi(t)) d \mathcal{W}(t), \quad \xi(0)=\xi_{0} .
$$

Here, $D_{\xi}(\sigma(\xi))$ denotes the Fréchet derivative of $\sigma$ with respect to $\xi$.

Let us assume that the Wiener process is cylindrical in $H^{\delta}(\mathcal{O}), \delta>1$. Then, the Wiener process is infinite dimensional and it can be written as a sum of infinitely many scalar Wiener 
processes, i.e. $\mathcal{W}(t, x)=\sum_{k \in \mathbb{Z}} \psi_{k}^{(\delta)}(x) \beta_{k}(t)$, where $\left\{\beta_{k}: k \in \mathbb{Z}\right\}$ is a family of independent scalar-valued Wiener processes and $\psi_{k}^{(\delta)}:=\left(1+(2 \pi k)^{2}\right)^{-\delta / 2} \psi_{k}$. Here $\left\{\psi_{k}: k \in \mathbb{Z}\right\}$ is the orthonormal basis given in (2.3). Elementary calculations reveal for $\sigma(\xi)=\xi$

$$
\sum_{k \in \mathbb{Z}} D_{\xi} \sigma(\xi)\left[\psi_{k}^{(\delta)}\right] \sigma(\xi)\left[\psi_{k}^{(\delta)}\right]=\sum_{k \in \mathbb{Z}} \xi \psi_{k}^{(\delta)} \psi_{k}^{(\delta)}
$$

We call $\sigma_{k}: H \rightarrow L_{H S}(H)$ by $\sigma_{k}(\xi)=\lambda_{k} \xi \psi_{k}$. Indeed, denoting $\left(1+(2 \pi k)^{2}\right)^{-\delta / 2}$ by $\lambda_{k}$ and taking into account the relations $\lambda_{k}=\lambda_{-k}, \psi_{k}^{2}+\psi_{-k}^{2}=2$ and $\gamma=\sum_{k \in \mathbb{Z}} \lambda_{k}^{2}$, we infer

$$
\begin{aligned}
\sum_{k \in \mathbb{Z}} D_{\xi} \sigma_{k}(\xi) \sigma_{k}(\xi) & =\sum_{k \in \mathbb{Z}} \xi \psi_{k}^{(\delta)} \psi_{k}^{(\delta)}=\sum_{k \in \mathbb{N}} \lambda_{k}^{2} \psi_{k} \psi_{k} \xi+\sum_{k \in \mathbb{N}} \lambda_{-k}^{2} \psi_{-k} \psi_{-k} \xi+\lambda_{0}^{2} \xi \\
& =2 \sum_{k \in \mathbb{N}} \lambda_{k}^{2} \xi+\lambda_{0}^{2} \xi=\sum_{k \in \mathbb{Z}} \lambda_{k}^{2} \xi=\gamma \xi
\end{aligned}
$$

We shortly give the exact form of the Burkholder-Davis-Gundy inequality which will be useful during the course of analysis. Given a Wiener process $\mathcal{W}$ being cylindrical on $\mathcal{H}:=H^{\delta}(\mathcal{O}), \delta>1$, over $\mathfrak{A}$, and a progressively measurable process $\xi \in M_{\mathfrak{A}}^{2}\left(0, T ; H^{\rho}(\mathcal{O})\right), \rho \in\left[0, \frac{1}{2}\right]$, let us define $\{Y(t): t \in[0, T]\}$ by

$$
Y(t):=\int_{0}^{t} \xi(s) d \mathcal{W}(s), \quad t \in[0, T]
$$

Here, for each $t \in[0, T], \xi(t)$ is interpreted as a multiplication operator acting on the elements of $\mathcal{H}$, namely, $\xi: \mathcal{H} \ni \psi \mapsto \xi \psi \in \mathcal{S}^{\prime}(\mathbb{R})$. Since for any $\nu>\frac{1}{2}$ and for any $\varphi \in H^{\nu}(\mathcal{O})$ the product $\xi(t) \varphi$ belongs to $H^{\rho}(\mathcal{O})$ (see [29, p. 190]), we can view $\xi(t)$ as a linear map from $H^{\nu}(\mathcal{O})$ into $H^{\rho}(\mathcal{O})$. It is shown in [29, Theorem $1(4)$, p. 190] that

$$
|\xi(t) \varphi|_{H^{\rho}} \leq C|\xi(t)|_{H^{\rho}}|\varphi|_{H^{\nu}}
$$

where the constant $C>0$ is independent of $\varphi$. Consequently, for any $p \geq 1, \delta>1$, and any $\rho \in\left[0, \frac{1}{2}\right]$

$$
\mathbb{E}\left[\sup _{t \in[0, T]}|Y(t)|_{H^{\rho}}^{p}\right] \leq C_{p} \mathbb{E}\left[\int_{0}^{T}|\xi(t)|_{L_{\mathrm{HS}}\left(H^{\delta}, H^{\rho}\right)}^{2} d t\right]^{\frac{p}{2}},
$$

where $|\cdot|_{L_{\mathrm{HS}}\left(H^{\delta}, H^{\rho}\right)}$ denotes the Hilbert-Schmidt norm from $H^{\delta}(\mathcal{O})$ to $H^{\rho}(\mathcal{O})$. First, let us note that for $\delta>1$ there exists a $\nu>\frac{1}{2}$ such that the embedding $H^{\delta}(\mathcal{O}) \hookrightarrow H^{\nu}(\mathcal{O})$ is a HilbertSchmidt operator. Using the fact that $\left\{\psi_{k}^{(\delta)}: k \in \mathbb{Z}\right\}$ is an orthonormal basis of $H^{\delta}(\mathcal{O})$, and $\psi_{k}^{(\delta)}=\lambda_{k} \psi_{k}$ we obtain, by using (3.5),

$$
|\xi(s)|_{L_{\mathrm{HS}}\left(H^{\delta}, H^{\rho}\right)}^{2}=\sum_{k \in \mathbb{Z}}\left|\xi(s) \psi_{k}^{(\delta)}\right|_{H^{\rho}}^{2} \leq C \sum_{k \in \mathbb{Z}}|\xi(s)|_{H^{\rho}}^{2}\left|\psi_{k}^{(\delta)}\right|_{H^{\nu}}^{2}=C|\xi(s)|_{H^{\rho}}^{2} \sum_{k \in \mathbb{Z}}\left|\psi_{k}^{(\delta)}\right|_{H^{\nu}}^{2}
$$

If the embedding $H^{\delta}(\mathcal{O}) \hookrightarrow H^{\rho}(\mathcal{O})$ is supposed to be a Hilbert-Schmidt, the right hand side of (3.6) is finite and we obtain

$$
\mathbb{E}\left[\sup _{t \in[0, T]}|Y(t)|_{H^{\rho}}^{p}\right] \leq C \mathbb{E}\left[\int_{0}^{T}|\xi(t)|_{H^{\rho}}^{2} d t\right]^{\frac{p}{2}}
$$


In case $\rho \geq \frac{1}{2}$, we use the fact that $H^{\rho}(\mathcal{O})$ is an algebra. Therefore, we obtain

$$
|\xi(s)|_{L_{\mathrm{HS}}\left(H^{\delta}, H^{\rho}\right)}^{2}=\sum_{k \in \mathbb{Z}}\left|\xi(s) \psi_{k}^{(\delta)}\right|_{H^{\rho}}^{2}=|\xi(s)|_{H^{\rho}}^{2} \sum_{k \in \mathbb{Z}}\left|\psi_{k}^{(\delta)}\right|_{H^{\rho}}^{2} .
$$

If the embedding $H^{\delta}(\mathcal{O}) \hookrightarrow H^{\rho}(\mathcal{O})$ is a Hilbert-Schmidt operator, then the right hand side of (3.8) is finite and we obtain for $\delta>\rho+1$

$$
\mathbb{E}\left[\sup _{t \in[0, T]}|Y(t)|_{H^{\rho}}^{p}\right] \leq C \mathbb{E}\left[\int_{0}^{T}|\xi(t)|_{H^{\rho}}^{2} d t\right]^{\frac{p}{2}} .
$$

For further details we refer to the linear noise (see [2, Example 2.1.2, p. 22]). After this short preparatory step we now start the proof.

\subsection{Proof of Main Result.}

Proof of Theorem [2.6. We show the existence of a solution to the system written in the Itô form, i.e., to

$$
\left\{\begin{array}{c}
d u-\left(r_{u} A u-\chi \operatorname{div}(u \nabla v)+\gamma u\right) d t=u d \mathcal{W}_{1} \\
d v-\left(r_{v} A v-(\alpha-\gamma) v\right) d t=\beta u d t+v d \mathcal{W}_{2}
\end{array}\right.
$$

Here, the stochastic differential is interpreted as the Itô stochastic differentials. Adding the correction term calculated in (3.4) we see that the equation (3.10) is equivalent to (2.2), where the stochastic integral is interpreted as the Stratonovich integral. The advantage of the Itô stochastic integral is, that the Itô integral is a local martingale and we can apply the BurkholderDavis-Gundy inequality. For simplicity we write $\alpha$ as $\alpha-\gamma$. In this way we end up with the following system

$$
\left\{\begin{array}{r}
d u-\left(r_{u} A u-\chi \operatorname{div}(u \nabla v)+\gamma u\right) d t=u d \mathcal{W}_{1} \\
d v-\left(r_{v} A v-\alpha v\right) d t=\beta u d t+v d \mathcal{W}_{2}
\end{array}\right.
$$

As mentioned before, we have at our disposal the Burkholder-Davis-Gundy maximal inequality. By the definition of $\gamma$ and the remark above (see Section 3.1) it follows that the systems (2.2) and (3.11) are equivalent.

The proof of Theorem 2.6 is carried out in several steps. First, we consider the truncated system and show that the solution of the truncated system exists depending on the truncation parameter $n$. The immediate next step is to achieve non-negativity of the solution, provided the initial conditions are. Then using appropriate Lyapunov functional, we obtain a uniform estimate in the $L^{1}$ norm of the family of solutions for the truncated system, by which we obtain the existence of a global solution.

Step I: The truncated system: Let $\psi \in C_{c}^{\infty}(\mathbb{R})$ be a cut-off function which satisfies

$$
\psi(x):= \begin{cases}0 & \text { if }|x| \geq 2, \\ \in[0,1] & \text { if }|x| \in(1,2), \\ 1 & \text { if }|x| \leq 1,\end{cases}
$$

and let $\psi_{n}(x):=\psi(x / n), x \in \mathbb{R}, n \in \mathbb{N}$. In addition, for any progressively measurable process $\eta_{1}$ and $\eta_{2}$ over $\mathfrak{A}$ belonging to $\mathcal{M}_{\mathfrak{A}}^{2}\left(0, T ; L^{1}(\mathcal{O})\right)$ and $\mathcal{M}_{\mathfrak{A}}^{2}\left(0, T ; H^{1}(\mathcal{O})\right)$ respectively, let us define 
for $t \in[0, T]$

$h^{1}\left(\eta_{1}, t\right):=\sup _{0 \leq s \leq t}\left|\eta_{1}(s)\right|_{L^{1}}, h^{2}\left(\eta_{2}, t\right):=\sup _{0 \leq s \leq t}\left|\nabla \eta_{2}(s)\right|_{L^{2}}$, and $h^{3}\left(\eta_{2}, t\right):=\int_{0}^{t}\left|\nabla \eta_{2}(s)\right|_{H^{1}}^{2} d s$.

Let us consider the truncated system given by

$$
\left\{\begin{aligned}
& d u_{n}(t)-\left[r_{u} A u_{n}(t)-\chi \psi_{n}\left(h^{1}\left(u_{n}, t\right)\right) \psi_{n}\left(h^{2}\left(v_{n}, t\right)\right) \psi_{n}\left(h^{3}\left(v_{n}, t\right)\right)\right. \\
&\left.\times \operatorname{div}\left(u_{n}(t) \nabla v_{n}(t)\right)+\gamma u_{n}(t)\right] d t=u_{n}(t) d \mathcal{W}_{1}(t) \\
& d v_{n}(t)-\left[r_{v} A v_{n}(t)-\alpha v_{n}(t)\right] d t=\beta u_{n}(t) d t+v_{n}(t) d \mathcal{W}_{2}(t) \\
&\left(u_{n}(0), v_{n}(0)\right)=\left(u_{0}, v_{0}\right)
\end{aligned}\right.
$$

First we show in the following lemma that this truncated system has a martingale solution $\left(u_{n}, v_{n}\right)$ (in the sense of Definition 2.2$)$ belonging $\mathbb{P}$-a.s. to $C_{b}^{0}\left([0, T] ; L^{2}(\mathcal{O})\right) \times C_{b}^{0}\left([0, T] ; H^{1}(\mathcal{O})\right.$ ).

Lemma 3.1. Let us assume that Assumption 2.3 is satisfied. For each $n \in \mathbb{N}$, and any initial condition $u_{0}, v_{0}$ satisfying Assumption 2.5, there exists a martingale solution $\left(\mathfrak{A}_{n},\left(\mathcal{W}_{1}^{n}, \mathcal{W}_{2}^{n}\right)\right.$, $\left.\left(u_{n}, v_{n}\right)\right)$, where $\mathfrak{A}_{n}=\left(\Omega_{n}, \mathcal{F}_{n},\left(\mathcal{F}_{t}^{n}\right)_{t \in[0, T]}, \mathbb{P}_{n}\right)$ is a filtered probability space and $\left(u_{n}, v_{n}\right)$ is a strong solution to the system (3.13) such that $\left(u_{n}, v_{n}\right)$ belongs $\mathbb{P}_{n}-$ a.s. to $C_{b}^{0}\left([0, T] ; L^{2}(\mathcal{O})\right) \times$ $C_{b}^{0}\left([0, T] ; H^{1}(\mathcal{O})\right)$. In addition, for all $n \in \mathbb{N}$, there exists a constant $C=C(n)>0$ such that

$$
\mathbb{E}^{n}\left[\sup _{0 \leq s \leq T}\left|u_{n}(s)\right|_{L^{2}}^{2}\right]+\mathbb{E}^{n}\left[\sup _{0 \leq s \leq T}\left|\nabla v_{n}(s)\right|_{L^{2}}^{2}\right] \leq C(n)
$$

The proof of Lemma 3.1 is carried out by applying the Schauder-Tychonoff Fixed Point Theorem to the mapping $\mathfrak{T}_{n}: \mathcal{M}_{\mathfrak{A}}^{2}\left(0, T ; L^{2}(\mathcal{O})\right) \rightarrow \mathcal{M}_{\mathfrak{A}}^{2}\left(0, T ; L^{2}(\mathcal{O})\right)$ defined for each $n \in \mathbb{N}$ as follows. Each process $\xi \in \mathcal{M}_{\mathfrak{A}}^{2}\left(0, T ; L^{2}(\mathcal{O})\right)$ is assigned a value $\mathfrak{T}_{n}(\xi):=u_{n}$ and another value $\mathfrak{R}(\xi):=v$ where $\left(u_{n}, v\right)$ is the unique solution to

$$
\left\{\begin{array}{l}
d u_{n}(t)-\left[r_{u} A u_{n}(t)-\chi \psi_{n}\left(h^{1}(\xi, t)\right) \psi_{n}\left(h^{2}(v, t)\right) \psi_{n}\left(h^{3}(v, t)\right)\right. \\
\left.\times \operatorname{div}(\xi(t) \nabla v(t))+\gamma u_{n}(t)\right] d t=u_{n}(t) d \mathcal{W}_{1}(t), \\
d v(t)-\left[r_{v} A v(t)-\alpha v(t)\right] d t=\beta \xi(t) d t+v(t) d \mathcal{W}_{2}(t), \\
\left(u_{n}(0), v(0)\right)=\left(u_{0}, v_{0}\right) .
\end{array}\right.
$$

First, let us check that the operators $\mathfrak{T}_{n}: \mathcal{M}_{\mathfrak{A}}^{2}\left(0, T ; L^{2}(\mathcal{O})\right) \rightarrow \mathcal{M}_{\mathfrak{A}}^{2}\left(0, T ; L^{2}(\mathcal{O})\right)$ and $\mathfrak{R}$ : $\mathcal{M}_{\mathfrak{A}}^{2}\left(0, T ; L^{2}(\mathcal{O})\right) \rightarrow \mathcal{M}_{\mathfrak{A}}^{2}\left(0, T ; L^{2}(\mathcal{O})\right)$ are well defined, namely we verify that there exists a unique solution $\left(u_{n}, v\right)$ to system (3.14). For each $\xi \in \mathcal{M}_{\mathfrak{A}}^{2}\left(0, T ; L^{2}(\mathcal{O})\right)$, the unique existence of $v$ belonging to $L^{\infty}\left(0, T ; H^{1}(\mathcal{O})\right) \cap L^{2}\left(0, T ; H^{2}(\mathcal{O})\right)$ can be shown by standard arguments, see e.g. [11, Theorem 7.2]. Note that the process $v$ is independent of the cut-off argument; therefore it is independent of $n \in \mathbb{N}$. Again, due to [11, Theorem 7.5, p. 197] given $v$ and $\xi$ we can conclude that there exists a unique solution $u_{n}$ to the first equation in (3.14). This ensures that the operators $\mathfrak{T}_{n}$ and $\mathfrak{R}$ are well defined.

Next, we investigate some properties of the operator $\mathfrak{T}_{n}$ which are necessary to formulate a Schauder-Tychnoff Fixed Point Theorem. In the following we will show that 
- there exists a convex subset $\mathcal{C} \subset \mathcal{M}_{\mathfrak{A}}^{2}\left(0, T ; L^{2}(\mathcal{O})\right)$ such that $\mathfrak{T}_{n}$ maps $\mathcal{C}$ into itself,

- $\mathfrak{T}_{n}$ maps bounded sets into precompact sets,

- $\mathfrak{T}_{n}$ restricted to bounded sets is continuous.

Using the compactness of the operator $\mathfrak{T}_{n}$ defined on a closed convex subset of $\mathcal{M}_{\mathfrak{A}}^{2}\left(0, T ; L^{2}(\mathcal{O})\right)$, we obtain a fixed point of $\mathfrak{T}_{n}$, which is a solution of (3.13). Since we use compactness and not a contraction principle, this step can be seen as a Schauder-Tychonoff Type Fixed Point Theorem.

Let us start with the first claim.

Claim 3.1. The following two statements hold.

(a) For any $n \in \mathbb{N}$ and any initial condition $\left(u_{0}, v_{0}\right)$ satisfying Assumption [2.5, there exists a number $R=R\left(n, u_{0}\right)>0$ such that $\mathfrak{T}_{n}: \mathcal{C}_{R} \longrightarrow \mathcal{C}_{R}$, where

$$
\mathcal{C}_{R}:=\left\{\xi \in \mathcal{M}_{\mathfrak{A}}^{2}\left(0, T ; L^{2}(\mathcal{O})\right):\|\xi\|_{\mathcal{M}_{\mathfrak{A}}\left(0, T ; L^{2}\right)}^{2} \leq R\right\} .
$$

In particular, $R$ is of the form $C(T)\left[\mathbb{E}\left|u_{0}\right|_{L^{2}}+(2 n)^{5 / 4}\right]$.

(b) For any $n \in \mathbb{N}$, any initial condition $\left(u_{0}, v_{0}\right)$ satisfying Assumption 2.5 , and for any $\bar{\alpha}, \beta \geq 0$ with $\bar{\alpha}+2 \beta<\frac{1}{2}$,

- there exists a number $r=r(\bar{\alpha}, T, n)>0$ such that for any $\xi \in \mathcal{M}_{\mathfrak{A}}^{2}\left(0, T ; L^{2}(\mathcal{O})\right)$ we have

$$
\sup _{0 \leq t \leq T} \mathbb{E}\left|\left(\mathfrak{T}_{n} \xi\right)(t)-e^{t\left(r_{u} A+\gamma I\right)} u_{0}\right|_{H^{\bar{\alpha}}}^{2} \leq r\|\xi\|_{\mathcal{M}_{\mathfrak{A}}^{2}\left(0, T ; L^{2}\right)}^{2}
$$

- there exists a number $\delta=\delta(\bar{\alpha}, \beta, T, n)>0$ and a number $C=C(\delta, T, n)>0$ such that for any $0<t_{1}<t_{2} \leq T$ and $\xi \in \mathcal{M}_{\mathfrak{A}}^{2}\left(0, T ; L^{2}(\mathcal{O})\right)$ we have

$$
\mathbb{E}\left|\left(\mathfrak{T}_{n} \xi\right)\left(t_{1}\right)-\left(\mathfrak{T}_{n} \xi\right)\left(t_{2}\right)\right|_{L^{2}}^{2} \leq C\left|t_{2}-t_{1}\right|^{\delta}\|\xi\|_{\mathcal{M}_{\mathfrak{A}}^{2}\left(0, T ; L^{2}\right)}^{2}
$$

Proof of Claim [3.1: Let us fix $\xi \in \mathcal{M}_{\mathfrak{A}}^{2}\left(0, T ; L^{2}(\mathcal{O})\right)$ and let $\left(u_{n}, v\right)$ be a solution to system (3.14). Firstly, we observe that by the variation of constant formula and the semigroup generated by $B=-r_{u} A-\gamma I$ with $D(B)=D(A)$, we have

$$
\begin{aligned}
\mathfrak{T}_{n} \xi(t)=u_{n}(t)= & e^{t\left(r_{u} A+\gamma I\right)} u_{0} \\
+ & \chi \int_{0}^{t} e^{(t-s)\left(r_{u} A+\gamma I\right)} \psi_{n}\left(h^{1}(\xi, t)\right) \psi_{n}\left(h^{2}(v, t)\right) \psi_{n}\left(h^{3}(v, s)\right) \operatorname{div}(\xi(t) \nabla v(s)) d s \\
& +\int_{0}^{t} e^{(t-s)\left(r_{u} A+\gamma I\right)} u_{n}(s) d \mathcal{W}_{1}(s), t \in[0, T] .
\end{aligned}
$$

Secondly, let us note that we have by the smoothing of the semigroup

$$
\left|e^{t\left(r_{u} A+\gamma I\right)} \operatorname{div} w\right|_{L^{2}} \leq\left(1+t^{-1 / 2}\right) e^{-\left(r_{u} \lambda_{1}+\gamma\right) t}|w|_{L^{2}}
$$

for any $w \in H^{1}(\mathcal{O})$, with $\lambda_{1}$ being the first eigenvalue of $A$. We define

$$
r(t):=\left(1+t^{-1 / 2}\right) e^{-\left(r_{u} \lambda_{1}+\gamma\right) t} .
$$

Next, for simplicity, let us introduce the abbreviations

$$
\Psi_{n}^{1}(s):=\psi_{n}\left(h^{1}(\xi, s)\right), \quad \Psi_{n}^{2}(s):=\psi_{n}\left(h^{2}(v, s)\right), \quad \text { and } \quad \Psi_{n}^{3}(s):=\psi_{n}\left(h^{3}(v, s)\right),
$$

where $h^{j}(\xi, s), j=1,2,3$, are defined in (3.12). Also, for simplicity, and since we will need this kind of calculations later on, we omit for the moment the cut-off function and derive a nice 
representation. Let us note that we have by [29, p. 238] and interpolation for $\varepsilon>\frac{1}{2}, \delta \in\left(0, \frac{1}{2}\right)$, $\rho=\delta+\frac{1}{2}$

$$
\begin{aligned}
& \int_{0}^{t}\left|e^{(t-s)\left(r_{u} A+\gamma I\right)} \operatorname{div}(\xi(s) \nabla v(s))\right|_{L^{2}} d s \\
= & \int_{0}^{t} r(t-s)|\xi(s) \nabla v(s)|_{L^{2}} d s \leq \int_{0}^{t} r(t-s)|\xi(s)|_{H^{-\delta}}|\nabla v(s)|_{H^{\rho}} d s \\
\leq & \int_{0}^{t} r(t-s)|\xi(s)|_{H^{-\varepsilon}}^{\delta / \varepsilon}|\xi(s)|_{L^{2}}^{1-\delta / \varepsilon}|\nabla v(s)|_{L^{2}}^{\frac{1}{2}-\delta}|\nabla v(s)|_{H^{1}}^{\delta+\frac{1}{2}} d s \\
\leq & C t^{\frac{5-2 \delta}{4}} \sup _{0 \leq s \leq t}|\xi(s)|_{H^{-\varepsilon}}^{\delta / \varepsilon} \sup _{0 \leq s \leq t}|\xi(s)|_{L^{2}}^{1-\delta / \varepsilon} \sup _{0 \leq s \leq t}|\nabla v(s)|_{L^{2}}^{\frac{1}{2}-\delta}\left(\int_{0}^{t}|\nabla v(s)|_{H^{1}}^{2} d s\right)^{\frac{\left(\delta+\frac{1}{2}\right)}{2}} .
\end{aligned}
$$

The constant $C$ depends on $\delta$.

We are now ready to prove item (a) in Claim 3.1, i.e., we find some $R>0$ such that $\mathfrak{T}_{n}$ maps $\mathcal{C}_{R}$ onto $\mathcal{C}_{R}$. Here, applying the consideration above, the Burkholder-Davis-Gundy inequality (3.7), and taking into account the cut-offs, we obtain

$$
\begin{aligned}
& \mathbb{E}\left[\sup _{0 \leq s \leq t}\left|u_{n}(s)\right|_{L^{2}}^{2}\right] \leq \mathbb{E}\left[\sup _{0 \leq s \leq t}\left|e^{s\left(r_{u} A+\gamma I\right)} u_{0}\right|_{L^{2}}^{2}\right]+\chi C t^{\frac{3-2 \delta}{4}} \mathbb{E}\left[\sup _{0 \leq s \leq t}\left(\Psi_{n}^{1}(s)\right)^{2}|\xi(s)|_{H^{-e p}}^{2 \delta / \varepsilon}\right. \\
& \left.\quad \times \sup _{0 \leq s \leq t}|\xi(s)|_{L^{2}}^{2-2 \delta / \varepsilon} \sup _{0 \leq s \leq t}\left(\Psi_{n}^{2}(s)\right)^{2}|\nabla v(s)|_{L^{2}}^{1-2 \delta}\left(\int_{0}^{t} \Psi_{n}^{3}(s)|\nabla v(s)|_{H^{1}}^{2} d s\right)^{2 \delta+1 / 2}\right] \\
& \quad+\mathbb{E}\left[\int_{0}^{t} \sup _{0 \leq r \leq s}\left|u_{n}(r)\right|_{L^{2}}^{2} d s\right] .
\end{aligned}
$$

Due to the definition of $\Psi_{n}^{1}, \Psi_{n}^{2}$, and $\Psi_{n}^{3}$ we can write

$$
\begin{aligned}
\mathbb{E}\left[\sup _{0 \leq s \leq t}\left|u_{n}(s)\right|_{L^{2}}^{2}\right] \leq \mathbb{E}\left[\sup _{0 \leq s \leq t}\left|e^{s\left(r_{u} A+\gamma I\right)} u_{0}\right|_{L^{2}}^{2}\right]+\chi C t^{\frac{7-2 \delta}{4}} \mathbb{E}\left[(2 n)^{2 \delta / \varepsilon} \sup _{0 \leq s \leq t}|\xi(s)|_{L^{2}}^{2(1-\delta / \varepsilon)}\right. \\
\times(2 n)^{1-2 \delta}(2 n)^{2 \delta+\frac{1}{2}}+\mathbb{E}\left[\int_{0}^{t} \sup _{0 \leq r \leq s}\left|u_{n}(r)\right|_{L^{2}}^{2} d s\right]
\end{aligned}
$$

Applying Gronwall's lemma we obtain

$$
\mathbb{E}\left[\sup _{0 \leq s \leq t}\left|u_{n}(s)\right|_{L^{2}}^{2}\right] \leq C(t) \mathbb{E}\left[\left|u_{0}\right|_{L^{2}}^{2}+\xi(2 n)^{2 \delta / \varepsilon+\frac{3}{2}}\left(\mathbb{E} \sup _{0 \leq s \leq t}|\xi(s)|_{L^{2}}^{2}\right)^{1-\delta / \varepsilon}\right] .
$$

Taking $\delta=\frac{\varepsilon}{2}$, it follows that if $R$ satisfies

$$
R>\sqrt{C(t)}\left[\mathbb{E}\left|u_{0}\right|_{L^{2}}+(2 n)^{\frac{5}{4}} \sqrt{R}\right]
$$

then $\mathfrak{T}_{n}(\xi) \in \mathcal{C}_{R}$. Therefore, for any $n \in \mathbb{N}$ there exists a number $R=R\left(n, u_{0}\right)>0$ given by (3.20) such that $\mathfrak{T}_{n}$ maps $\mathcal{C}_{R}$ to $\mathcal{C}_{R}$. 
To show item (b) in Claim [3.1, we use the following result. Suppose that $t>s$ and $\eta \in$ $L^{q}\left(0, T ; L^{2}(\mathcal{O})\right)$. Let

$$
\left(\mathfrak{F}_{r_{u} A+\gamma I} \eta\right)(t)=\int_{0}^{t} e^{(t-s)\left(r_{u} A+\gamma I\right)} \eta(s) d s, \quad t \in[0, T] .
$$

In Lemma 2.4 of [8], compare also with Lemma A.3 in Appendix A, it is shown that, for $\rho>\frac{1}{4}$ and $\beta>0$, if $\beta<1-\rho-\frac{1}{q}-\bar{\alpha} / 2$ and $\eta \in L^{q}\left(0, T ; H^{-\rho}(\mathcal{O})\right)$, then $\mathfrak{F}_{r_{u} A+\gamma I} \eta \in C^{\beta}\left([0, T] ; H^{\bar{\alpha}}(\mathcal{O})\right)$. Moreover,

$$
\left\|\mathfrak{F}_{r_{u} A+\gamma I} \eta\right\|_{C^{\beta}\left([0, T] ; H^{\bar{\alpha}}\right)} \leq C\|\eta\|_{L^{q}\left(0, T ; H^{-2 \rho}\right)} .
$$

Let us put $\eta(s)=\phi_{n}\left(h^{1}(\xi, s)\right) \phi_{n}\left(h^{2}(v, s)\right) \phi_{n}\left(h^{3}(v, s)\right) \operatorname{div}(\xi(s) \nabla v(s)), s \in[0, T]$. Then, by the Sobolev embedding $L^{1}(\mathcal{O}) \hookrightarrow H^{-(2 \rho+1)}(\mathcal{O})$ we obtain

$$
|\operatorname{div}(\xi(s) \nabla v(s))|_{H^{-2 \rho}} \leq|\xi(s) \nabla v(s)|_{H^{-(2 \rho+1)}} \leq|\xi(s) \nabla v(s)|_{L^{1}} \leq|\xi(s)|_{L^{2}}|\nabla v(s)|_{L^{2}} .
$$

Due to the definitions of the $h^{2}(v, \cdot), h^{3}(v, \cdot)$, and $\eta$ we have for and $q \geq 2$

$$
\|\eta\|_{L^{q}\left(0, T ; H^{-2 \rho}\right)} \leq 2 n \sup _{0 \leq s \leq T}|\xi(s)|_{L^{2}}
$$

Hence, $\mathfrak{F}_{r_{u} A+\gamma I} \eta \in C_{b}^{\beta}\left([0, T] ; H^{\bar{\alpha}}(\mathcal{O})\right)$ for $\bar{\alpha}, \beta \geq 0$ and $\bar{\alpha}+2 \beta<\frac{1}{2}$.

It remains to show the continuity for the stochastic term. Recalling that $\xi \in \mathcal{M}_{\mathfrak{A}}^{2}\left(0, T ; L^{2}(\mathcal{O})\right)$ we define

$$
\left(\mathfrak{S}_{r_{u} A+\gamma I} \xi\right)(t)=\int_{0}^{t} e^{(t-s)\left(r_{u} A+\gamma I\right)} \xi(s) d \mathcal{W}_{1}(s), \quad t \in[0, T] .
$$

We first show that there exists $C>0$ such that

$$
\mathbb{E}\left[\sup _{0<t \leq T}\left|\mathfrak{S}_{r_{u} A+\gamma I} \xi\right|_{H^{\alpha}}\right] \leq C\|\xi\|_{\mathcal{M}_{\mathfrak{A}}^{2}\left(0, T ; L^{2}\right)} .
$$

Indeed, choosing $\beta=0, \delta=\bar{\alpha} / 2, \rho=0$, and $p>1$ such that $\bar{\alpha} / 2+1 / p<1 / 2$ in Lemma A.3, we deduce

$$
\mathbb{E}\left[\left|\mathfrak{S}_{r_{u} A+\gamma I} \xi\right|_{C_{b}^{0}\left([0, T] ; H^{\bar{\alpha}}(\mathcal{O})\right)}^{p}\right] \leq C \mathbb{E}\left[\int_{0}^{T}|\xi(s)|_{L_{\mathrm{HS}}\left(H, L^{2}(\mathcal{O})\right)}^{p} d s\right] \leq C_{T} \mathbb{E}\left[\sup _{0 \leq s \leq T}\|\xi(s)\|_{L^{2}(\mathcal{O})}^{p}\right] .
$$

This essentially yields (3.21). Elementary calculations reveal

$$
\mathbb{E}\left|\int_{0}^{t} e^{(t-s)\left(r_{u} A+\gamma I\right)} \xi(s) d \mathcal{W}_{1}(s)\right|_{H^{\alpha}}^{2} \leq C_{\alpha} \mathbb{E}\left[\int_{0}^{t}(t-s)^{-\alpha}|\xi(s)|_{L^{2}}^{2} d s\right] \leq \frac{t^{1-\alpha}}{1-\alpha}\|\xi\|_{\mathcal{M}_{\mathfrak{l}}^{2}\left(0, T ; L^{2}\right)}^{2} .
$$

For $T \geq t>s>0$, elementary calculations lead to the following identity

$$
\begin{aligned}
& \left(\mathfrak{S}_{r_{u} A+\gamma I} \xi\right)(t)-\left(\mathfrak{S}_{r_{u} A+\gamma I} \xi\right)(s)=\int_{0}^{t} e^{(t-r)\left(r_{u} A+\gamma I\right)} \xi(r) d \mathcal{W}_{1}(r)-\int_{0}^{s} e^{(s-r)\left(r_{u} A+\gamma I\right)} \xi(r) d \mathcal{W}_{1}(r) \\
& =\int_{s}^{t} e^{(t-r)\left(r_{u} A+\gamma I\right)} \xi(r) d \mathcal{W}_{1}(r)+\left(e^{(t-s)\left(r_{u} A+\gamma I\right)}-I\right) \int_{0}^{s} e^{(s-r)\left(r_{u} A+\gamma I\right)} \xi(r) d \mathcal{W}_{1}(r) \\
& =\int_{s}^{t} e^{(t-r)\left(r_{u} A+\gamma I\right)} \xi(r) d \mathcal{W}_{1}(r)+\left(e^{(t-s)\left(r_{u} A+\gamma I\right)}-I\right)\left(\mathfrak{S}_{r_{u} A+\gamma I} \xi\right)(s) .
\end{aligned}
$$


Then, it follows from Remark 1.1 [16] that

$\mathbb{E}\left[\sup _{s \leq t \leq s+h}\left|\int_{s}^{t} e^{-(t-r)\left(r_{u} A+\gamma I\right)} \xi(r) d \mathcal{W}_{1}(r)\right|_{H^{\bar{\alpha}}}^{2}\right] \leq h^{-\bar{\alpha}} \mathbb{E}\left(\int_{s}^{s+h}|\xi(s)|_{L^{2}}^{2} d s\right)^{\frac{p}{2}} \leq\|\xi\|_{\mathcal{M}_{\mathfrak{l}}^{2}\left(0, T ; L^{2}\right)}^{p} h^{\frac{p}{2}}$.

In addition, by standard calculations we know

$$
\left|\left(e^{(t-s)\left(r_{u} A+\gamma I\right)}-I\right)\left(\mathfrak{S}_{r_{u} A+\gamma I} \xi\right)(s)\right|_{L^{2}}^{2} \leq(t-s)^{\bar{\alpha}}\left|\left(\mathfrak{S}_{r_{u} A+\gamma I} \xi\right)(s)\right|_{H^{\bar{\alpha}}}^{2}
$$

Due to estimate (3.21) there exists a number $\bar{\alpha}>0$ such that

$$
\mathbb{E}\left[\sup _{0<s<t \leq T}\left|\left(\mathfrak{S}_{r_{u} A+\gamma I} \xi\right)(t)-\left(\mathfrak{S}_{r_{u} A+\gamma I} \xi\right)(s)\right|_{H^{\bar{\alpha}}}^{2}\right] \leq C(t-s)^{\bar{\alpha}}\|\xi\|_{\mathcal{M}_{\mathfrak{I}}^{2}\left(0, T ; L^{2}\right)}^{2}
$$

This completes the proof of Claim 3.1 .

Next, we show the continuity of the mapping $\mathfrak{T}_{n}$.

Claim 3.2. Under Assumption 2.3, for any initial condition $\left(u_{0}, v_{0}\right)$ satisfying Assumption [2.5 and for any $R>0$, the mapping $\mathfrak{T}_{n}: \mathcal{C}_{R} \rightarrow \mathcal{M}_{\mathfrak{A}}^{2}\left(0, T ; L^{2}(\mathcal{O})\right)$ is continuous for each $n \in \mathbb{N}$, where $\mathcal{C}_{R}$ is defined by (3.15).

Proof of Claim 3.2: Let us fix $n \in \mathbb{N}$ and consider $\xi^{j} \in \mathcal{M}_{\mathfrak{A}}^{2}\left(0, T ; L^{2}(\mathcal{O})\right), j=1,2$. In order to prove the continuity of $\mathfrak{T}_{n}$, we will show that there exists a constant $C=C(n)>0$ such that

$$
\left\|\mathfrak{T}_{n}\left(\xi^{1}\right)-\mathfrak{T}_{n}\left(\xi^{2}\right)\right\|_{M_{\mathfrak{A}}^{2}\left(0, T ; L^{2}\right)} \leq C\left\|\xi^{1}-\xi^{2}\right\|_{M_{\mathfrak{l}}^{2}\left(0, T ; L^{2}\right)}^{2},
$$

where the $M_{\mathfrak{A}}^{2}\left(0, T ; L^{2}(\mathcal{O})\right)$-norm is defined by (3.1).

For $j=1,2$, let $\left(u_{n}^{j}, v^{j}\right)=\left(\mathfrak{T}_{n}\left(\xi^{j}\right), \mathfrak{R}\left(\xi^{j}\right)\right)$ be defined by (3.14) with $\xi$ replace by $\xi^{j}$. We define $\Psi_{n, j}^{1}, \Psi_{n, j}^{2}$, and $\Psi_{n, j}^{3}$ by (3.18) with $\xi$ and $v$ replaced by $\xi^{j}$ and $v^{j}$, respectively, $j=1,2$. Then it follows from the definition of $h^{j}$ in (3.12) that

$$
\begin{aligned}
\left|\Psi_{n, 1}^{1}(s)-\Psi_{n, 2}^{1}(s)\right| & =\left|\psi_{n}\left(h^{1}\left(\xi^{1}, s\right)\right)-\psi_{n}\left(h^{1}\left(\xi^{2}, s\right)\right)\right| \leq\left|\psi^{\prime}\right|_{L^{\infty}} \frac{1}{n}\left|h^{1}\left(v^{1}, s\right)-h^{1}\left(v^{2}, s\right)\right| \\
& \leq\left|\psi^{\prime}\right|_{L^{\infty}} \frac{1}{n} \sup _{0 \leq r \leq s}\left|\xi^{1}(r)-\xi^{2}(r)\right|_{L^{1}} .
\end{aligned}
$$

Next, we have

$$
\begin{aligned}
\left|\Psi_{n, 1}^{2}(s)-\Psi_{n, 2}^{2}(s)\right| & =\left|\psi_{n}\left(h^{2}\left(v^{1}, s\right)\right)-\psi_{n}\left(h^{2}\left(v^{2}, s\right)\right)\right| \leq\left|\psi^{\prime}\right|_{L^{\infty}} \frac{1}{n}\left|h^{2}\left(v^{1}, s\right)-h^{2}\left(v^{2}, s\right)\right| \\
& \leq\left|\psi^{\prime}\right|_{L^{\infty}} \frac{1}{n} \sup _{0 \leq r \leq s}\left|\nabla v^{1}(r)-\nabla v^{2}(r)\right|_{L^{2}}
\end{aligned}
$$

By similar calculations we obtain

$$
\left|\Psi_{n, 1}^{3}(s)-\Psi_{n, 2}^{3}(s)\right| \leq\left|\psi^{\prime}\right|_{L^{\infty}} \frac{1}{n} \int_{0}^{s}\left|\nabla v^{1}(r)-\nabla v^{2}(r)\right|_{H^{1}}^{2} d r
$$

The supremum on the right-hand side of (3.24) can be estimated by using the BurkholderDavis-Gundy inequality (3.9) as follows

$$
\mathbb{E}\left[\sup _{0 \leq s \leq t}\left|\nabla v^{1}(s)-\nabla v^{2}(s)\right|_{L^{2}}^{2}\right]
$$




$$
\begin{aligned}
& \leq \mathbb{E}\left[\sup _{0 \leq s \leq t}\left(\int_{0}^{s}\left(s-s_{1}\right)^{-\frac{1}{2}}\left|\xi_{1}\left(s_{1}\right)-\xi_{2}\left(s_{1}\right)\right|_{L^{2}} d s_{1}\right)^{2}\right]+\mathbb{E}\left[\sup _{0 \leq t \leq T} \int_{0}^{t}\left|\nabla\left(v^{1}(s)-v^{2}(s)\right)\right|_{L^{2}}^{2} d s\right] \\
& \leq 4 T \mathbb{E}\left[\sup _{0 \leq s \leq T}\left|\xi_{1}(s)-\xi_{2}(s)\right|_{L^{2}}^{2}\right]+\int_{0}^{t} \mathbb{E}\left[\sup _{0 \leq r \leq s}\left|\nabla\left(v^{1}(r)-v^{2}(r)\right)\right|_{L^{2}}^{2}\right] d s .
\end{aligned}
$$

Applying Gronwall's Lemma yields

$$
\mathbb{E}\left[\sup _{0 \leq s \leq t}\left|\nabla\left(v^{1}(s)-v^{2}(s)\right)\right|_{L^{2}}^{2}\right] \leq 4 T \mathbb{E}\left[\sup _{0 \leq s \leq t}\left|\xi_{1}(s)-\xi_{2}(s)\right|_{L^{2}}^{2}\right]
$$

Finally, the maximum regularity and the Burkholder-Davis-Gundy inequality given in (3.9) yield

$$
\mathbb{E}\left[\int_{0}^{t}\left|\nabla v^{1}(r)-\nabla v^{2}(r)\right|_{H^{1}}^{2} d r\right] \leq \mathbb{E}\left[\int_{0}^{t}\left|\xi^{1}(s)-\xi^{2}(s)\right|_{L^{2}}^{2} d s\right] \leq T \mathbb{E}\left[\sup _{0 \leq s \leq T}\left|\xi^{1}(s)-\xi^{2}(s)\right|_{L^{2}}^{2}\right] .
$$

In order to prove (3.22), we estimate the difference $\phi(t):=\mathfrak{T}_{n} \xi^{1}(t)-\mathfrak{T}_{n} \xi^{2}(t)=u_{n}^{1}(t)-u_{n}^{2}(t)$. It follows from the variation of constant formula (compare with (3.16)) that

$$
\begin{aligned}
\phi(t)= & \int_{0}^{t} e^{(t-s)\left(r_{u} A+\gamma I\right)}\left[\left(\Psi_{n, 1}^{1}(s) \Psi_{n, 1}^{2}(s) \Psi_{n, 1}^{3}(s) \operatorname{div}\left(\xi^{1}(s) \nabla v^{1}(s)\right)\right.\right. \\
& \left.-\Psi_{n, 2}^{1}(s), \Psi_{n, 2}^{2}(s) \Psi_{n, 1}^{3}(s) \operatorname{div}\left(\xi^{2}(s) \nabla v^{2}(s)\right)\right] d s+\int_{0}^{t} e^{(t-s)\left(r_{u} A+\gamma I\right)} \phi(s) d \mathcal{W}_{1}(s) \\
= & \underbrace{\int_{0}^{t} e^{(t-s)\left(r_{u} A+\gamma I\right)} \operatorname{div}\left(\left(\xi^{1}(s) \Psi_{n, 1}^{1}(s)-\xi^{2}(s) \Psi_{n, 2}^{1}(s)\right) \Psi_{n, 1}^{2}(s) \Psi_{n, 1}^{3}(s) \nabla v^{1}(s)\right) d s}_{I_{1}:=} \\
+ & \underbrace{\int_{0}^{t} e^{(t-s)\left(r_{u} A+\gamma I\right)} \Psi_{n, 2}^{1}(s) \operatorname{div}\left(\xi^{2}(s)\right)\left(\Psi_{n, 1}^{2}(s) \Psi_{n, 1}^{3}(s) \nabla v^{1}(s)-\Psi_{n, 2}^{2}(s) \Psi_{n, 2}^{3}(s) \nabla v^{2}(s)\right) d s}_{I_{2}:=} \\
& +\underbrace{\int_{0}^{t} e^{(t-s)\left(r_{u} A+\gamma I\right)}\left(\xi^{1}(s)-\xi^{2}(s)\right) d \mathcal{W}_{1}(s)}_{I_{3}:=} .
\end{aligned}
$$

Firstly, let us consider $I_{1}$. Let $\sigma \in\left(\frac{1}{2}, \frac{3}{4}\right), \delta \in\left(0, \frac{1}{2}\right)$ and $\rho=\delta+\frac{1}{2}$ be fixed. It is shown in 29, p. 238] that

$$
\left|\xi_{1} \xi_{2}\right|_{L^{2}} \leq\left|\xi_{1}\right|_{H^{-\delta}}\left|\xi_{2}\right|_{H^{\rho}, \quad \xi_{1} \in H^{-\delta}(\mathcal{O}), \xi_{2} \in H^{\rho}(\mathcal{O})}
$$

For any $\varepsilon \in\left(\frac{1}{2}, \frac{3}{4}\right)$, interpolation between $H^{-\varepsilon}(\mathcal{O})$ and $L^{2}(\mathcal{O})$ yields

$$
\left|\xi_{1}\right|_{H^{-\delta}} \leq\left|\xi_{1}\right|_{H^{-\varepsilon}}^{\delta / \varepsilon}\left|\xi_{1}\right|_{L^{2}}^{1-\delta / \varepsilon} \leq\left|\xi_{1}\right|_{L^{1}}^{\delta / \varepsilon}\left|\xi_{1}\right|_{L^{2}}^{1-\delta / \varepsilon}, \quad \xi_{1} \in L^{2}(\mathcal{O})
$$

Proceeding in similar lines as in (3.19), we recall the definition of $r$ in (3.17). Therefore, from the definition of the cut-off function and the smoothing property of the semigroup, we deduce 
that

$$
\begin{aligned}
\mathbb{E}\left[\sup _{0 \leq s \leq t}\left|I_{1}(s)\right|_{L^{2}}^{2}\right] \leq & \mathbb{E}\left[\sup _{0 \leq s \leq t} \mid \int_{0}^{s} e^{\left(s-s_{1}\right)\left(r_{u} A+\gamma I\right)} \Psi_{n, 1}^{2}\left(s_{1}\right) \Psi_{n, 1}^{3}\left(s_{1}\right)\right. \\
& \left.\times\left.\operatorname{div}\left(\left(\Psi_{n, 1}^{1}\left(s_{1}\right) \xi^{1}\left(s_{1}\right)-\Psi_{n, 2}^{1}\left(s_{1}\right) \xi^{2}\left(s_{1}\right)\right) \nabla v^{1}\left(s_{1}\right)\right) d s_{1}\right|_{L^{2}} ^{2}\right] \\
\leq & \mathbb{E}\left[\operatorname { s u p } _ { 0 \leq s \leq t } \left(\int_{0}^{s} r\left(s-s_{1}\right)\left|\Psi_{n, 1}^{1}\left(s_{1}\right) \xi^{1}\left(s_{1}\right)-\Psi_{n, 2}^{1}\left(s_{1}\right) \xi^{2}\left(s_{1}\right)\right|_{H^{-\delta}}\right.\right. \\
& \left.\left.\times \Psi_{n, 1}^{2}\left(s_{1}\right) \Psi_{n, 1}^{3}\left(s_{1}\right)\left|\nabla v^{1}\left(s_{1}\right)\right|_{H^{\rho}}^{2} d s_{1}\right)^{2}\right] \\
\leq & C t^{\frac{5-2 \delta}{4}} \mathbb{E}\left[\sup _{0 \leq s_{1} \leq t}\left|\Psi_{n, 1}^{1}\left(s_{1}\right) \xi^{1}\left(s_{1}\right)-\Psi_{n, 2}^{1}\left(s_{1}\right) \xi^{2}\left(s_{1}\right)\right|_{H^{-\delta}}\right. \\
& \left.\times\left(\int_{0}^{t}\left(\Psi_{n, 1}^{2}\left(s_{1}\right)\right)^{\frac{q}{2}}\left(\Psi_{n, 1}^{3}\left(s_{1}\right)\right)^{\frac{q}{2}}\left|\nabla v^{1}\left(s_{1}\right)\right|_{H^{\rho}}^{q} d s_{1}\right)^{\frac{2}{q}}\right] .
\end{aligned}
$$

In order to estimate the term involving the integral on the right-hand side of (3.30), we observe that due to interpolation $H^{1} \subset H^{\rho} \subset L^{2}$, we get for $0 \leq s_{1} \leq t$,

$$
\left|\nabla v^{1}\left(s_{1}\right)\right|_{H^{\rho}} \leq C\left|\nabla v^{1}\left(s_{1}\right)\right|_{L^{2}}^{\theta}\left|\nabla v^{1}\left(s_{1}\right)\right|_{H^{1}}^{1-\theta},
$$

with $\theta=1-\rho$ and $C>0$ is the interpolation constant depending on the dimension $\mathrm{d}$ and $\rho$. It follows for $q=\frac{4}{1+2 \delta}$

$$
\begin{aligned}
& \left(\int_{0}^{t}\left(\Psi_{n, 1}^{2}\left(s_{1}\right)\right)^{\frac{q}{2}}\left(\Psi_{n, 1}^{3}\left(s_{1}\right)\right)^{\frac{q}{2}}\left|\nabla v^{1}\left(s_{1}\right)\right|_{H^{\rho}}^{q} d s_{1}\right)^{\frac{2}{q}} \\
& \left.\leq C^{2} \sup _{0 \leq s \leq t}\left(\Psi_{n, 1}^{2}\left(s_{1}\right)\right)\left|\nabla v^{1}\left(s_{1}\right)\right|_{L^{2}}^{2 \theta}\right)\left(\int_{0}^{t}\left(\Psi_{n, 1}^{3}\left(s_{1}\right)\right)^{\frac{q}{2}}\left|\nabla v^{1}\left(s_{1}\right)\right|_{H^{1}}^{(1-\theta) q} d s_{1}\right)^{\frac{2}{q}} \\
& \left.\leq \theta C^{\frac{2}{\theta}} \sup _{0 \leq s \leq t}\left(\Psi_{n, 1}^{2}\left(s_{1}\right)\right)\left|\nabla v^{1}\left(s_{1}\right)\right|_{L^{2}}^{2 \theta}\right)^{\frac{1}{\theta}}+(1-\theta)\left(\int_{0}^{t}\left(\Psi_{n, 1}^{3}\left(s_{1}\right)\right)^{\frac{q}{2}}\left|\nabla v^{1}\left(s_{1}\right)\right|_{H^{1}}^{2} d s_{1}\right)^{\frac{2(1-\theta)}{q}} \\
& \leq(1-\rho) C^{\frac{2}{1-\rho}} \sup _{0 \leq s \leq t}\left(\left(\Psi_{n, 1}^{2}\left(s_{1}\right)\right)^{\frac{1}{1-\rho}}\left|\nabla v^{1}\left(s_{1}\right)\right|_{L^{2}}^{2}\right)+\rho\left(\int_{0}^{t}\left(\Psi_{n, 1}^{3}\left(s_{1}\right)\right)^{\frac{q}{2}}\left|\nabla v^{1}\left(s_{1}\right)\right|_{H^{1}}^{2} d s_{1}\right) .
\end{aligned}
$$

Using the definition of $\Psi_{n, 1}^{2}$, we obtain

$$
\Psi_{n, 1}^{2}\left(s_{1}\right)= \begin{cases}1 & \text { if } \sup _{0 \leq s_{1} \leq s}\left|\nabla v^{1}\left(s_{1}\right)\right|_{L^{2}}^{2} \leq n, \\ \in(0,1) & \text { if } \sup _{0 \leq s_{1} \leq s}\left|\nabla v^{1}\left(s_{1}\right)\right|_{L^{2}}^{2} \in(n, 2 n), \\ 0 & \text { otherwise. }\end{cases}
$$

Therefore, $\Psi_{n, 1}^{2}\left(s_{1}\right)\left|\nabla v^{1}\left(s_{1}\right)\right|_{L^{2}}^{2} \leq 2 n$. This eventually implies that

$$
\left(\Psi_{n, 1}^{2}\left(s_{1}\right)\right)^{\frac{1}{1-\rho}}\left|\nabla v^{1}\left(s_{1}\right)\right|_{L^{2}}^{2} \leq \Psi_{n, 1}^{2}\left(s_{1}\right)\left|\nabla v^{1}\left(s_{1}\right)\right|_{L^{2}}^{2}\left(\Psi_{n, 1}^{2}\left(s_{1}\right)\right)^{\frac{\rho}{\rho-1}} \leq 2 n .
$$

In similar manner, using the definition of $\Psi_{n, 1}^{3}$, one obtain that

$$
\int_{0}^{t}\left(\Psi_{n, 1}^{3}\left(s_{1}\right)\right)^{\frac{q}{2}}\left|\nabla v^{1}\left(s_{1}\right)\right|_{H^{1}}^{2} d s_{1} \leq 2 n
$$


Using (3.32)-(3.33) in (3.31) we obtain

$$
\left(\int_{0}^{t}\left(\Psi_{n, 1}^{2}\left(s_{1}\right)\right)^{\frac{q}{2}}\left(\Psi_{n, 1}^{3}\left(s_{1}\right)\right)^{\frac{q}{2}}\left|\nabla v^{1}\left(s_{1}\right)\right|_{H^{\rho}}^{q} d s_{1}\right)^{\frac{2}{q}} \leq 2 n(1-\rho) C^{\frac{2}{1-\rho}}+2 n \rho .
$$

We now estimate the term involving the supremum on the right-hand side of (3.30). Let

$$
\tau_{j}^{u}:=\inf _{s>0}\left\{\left|\xi^{j}(s)\right|_{L^{1}} \geq n\right\} \wedge T, \quad \tau_{j}^{o}:=\inf _{s>0}\left\{\left|\xi^{j}(s)\right|_{L^{1}} \geq 2 n\right\} \wedge T, j=1,2 .
$$

We distinguish the following cases:

$$
\left|\xi^{1}(s) \Psi_{n, 1}^{1}(s)-\xi^{2}(s) \Psi_{n, 2}^{1}(s)\right|_{H^{-\delta}} \leq\left\{\begin{array}{cl}
(2 n)^{\delta / \varepsilon}\left|\xi^{1}(s)-\xi^{2}(s)\right|_{L^{2}}^{1-\delta / \varepsilon} & \text { if } 0 \leq s \leq \underline{\tau}^{u}, \\
2^{\delta / \varepsilon} n^{\delta / \varepsilon-1}\left|\psi^{\prime}\right|_{L^{\infty}}\left|\xi^{1}(s)\right|_{L^{2}}^{1-\delta / \varepsilon} & \\
\times \sup _{0 \leq r \leq s}\left|\xi^{1}(r)-\xi^{2}(r)\right|_{L^{1}} & \\
+(4 n)^{\delta / \varepsilon}\left|\xi^{1}(s)-\xi^{2}(s)\right|_{L^{2}}^{1-\delta / \varepsilon} & \text { if } \underline{\tau}^{u}<s \leq \bar{\tau}^{o}, \\
0 & \text { if } s>\bar{\tau}^{o},
\end{array}\right.
$$

where $\underline{\tau}^{u}:=\min \left(\tau_{1}^{u}, \tau_{2}^{u}\right)$ and $\bar{\tau}^{o}:=\max \left(\tau_{1}^{o}, \tau_{2}^{o}\right)$. Elementary calculations reveal that

$$
\left(\int_{0}^{s}\left(r\left(s-s_{1}\right)\right)^{\frac{q}{q-1}} d s_{1}\right)^{\frac{2(q-1)}{q}} \leq 2\left(\frac{3-2 s}{1-2 s}\right)\left(s^{\frac{3-2 \delta}{2}}+s^{\frac{1-2 \delta}{2}}\right) .
$$

Substituting (3.31) and (3.34) into (3.30), using (3.35), and taking into account the fact that $\underline{\tau}^{u} \leq T$, we obtain

$$
\begin{aligned}
\mathbb{E}\left[\sup _{0 \leq s \leq t}\left|I_{1}(s)\right|_{L^{2}}^{2}\right] \leq & 2 C n\left(\frac{3-2 \delta}{1-2 \delta}\right)\left((1-\rho) C^{\frac{2}{1-\rho}}+\rho\right)\left(t^{\frac{3-2 \delta}{2}}+t^{\frac{1-2 \delta}{2}}\right) \\
& \times\left\{\mathbb{E}\left[\sup _{0 \leq s \leq \tau^{u}}(2 n)^{2 \delta / \varepsilon}\left|\xi^{1}(s)-\xi^{2}(s)\right|_{L^{2}}^{2(1-\delta / \varepsilon)}\right]\right. \\
+ & 2 \mathbb{E}\left[\operatorname { s u p } _ { \tau ^ { u } \leq s \leq \overline { \tau } ^ { o } } \left[2^{2 \delta / \varepsilon} n^{2(\delta / \varepsilon-1)}\left|\psi^{\prime}\right|_{L^{\infty}}^{2}\left|\xi^{1}(s)-\xi^{2}(s)\right|_{L^{1}}^{2}\left|\xi^{1}(s)\right|_{L^{2}}^{2(1-\delta / \varepsilon)}\right.\right. \\
& \left.\left.\left.+(4 n)^{2 \delta / \varepsilon}\left|\xi^{1}(s)-\xi^{2}(s)\right|_{L^{2}}^{2(1-\delta / \varepsilon)}\right]\right]\right\} \\
\leq & 2^{2 \delta / \varepsilon} 8 C\left(\frac{3-2 \delta}{1-2 \delta}\right)\left((1-\rho) C^{\frac{2}{1-\rho}}+\rho\right) n^{1+2 \delta / \varepsilon}\left(t^{\frac{3-2 \delta}{2}}+t^{\frac{1-2 \delta}{2}}\right) \\
& \times\left\{\left(\mathbb{E}\left[\sup _{0 \leq s \leq T}\left|\xi^{1}(s)-\xi^{2}(s)\right|_{L^{2}}^{2}\right]\right)^{(1-\delta / \varepsilon)}\right. \\
& +\frac{1}{n^{2}} R^{1-\delta / \varepsilon}\left(\mathbb{E}\left[\sup _{0 \leq s \leq T}\left|\xi^{1}(s)-\xi^{2}(s)\right|_{L^{2}}^{2 \varepsilon / \delta}\right)^{\delta / \varepsilon}\right\}
\end{aligned}
$$


We now treat the term $I_{2}$. We fix again $\delta \in\left(0, \frac{1}{2}\right), \rho=\delta+\frac{1}{2}$, and $\varepsilon \in\left(\frac{1}{2}, \frac{3}{4}\right)$. Using the same approach as in the calculation of (3.19) we obtain

$$
\begin{aligned}
\mathbb{E}\left[\sup _{0 \leq s \leq t}\left|I_{2}(s)\right|_{L^{2}}^{2}\right] \leq & \mathbb{E}\left[\sup _{0 \leq s \leq t} \mid \int_{0}^{s} e^{\left(s-s_{1}\right)\left(r_{u} A+\gamma I\right)} \Psi_{n, 2}^{1}\left(s_{1}\right)\right. \\
& \times \operatorname{div}\left(\left.\xi^{2}\left(s_{1}\right)\left[\Psi_{n, 1}^{2}\left(s_{1}\right) \Psi_{n, 1}^{3}\left(s_{1}\right) \nabla v^{1}\left(s_{1}\right)-\Psi_{n, 2}^{2}\left(s_{1}\right) \Psi_{n, 2}^{3}\left(s_{1}\right) \nabla v^{2}\left(s_{1}\right)\right] d s_{1}\right|_{L^{2}} ^{2}\right] \\
\leq & \mathbb{E}\left[\operatorname { s u p } _ { 0 \leq s \leq t } \left(\int_{0}^{s} r\left(s-s_{1}\right) \Psi_{n, 2}^{1}\left(s_{1}\right)\right.\right. \\
& \left.\left.\times\left|\xi^{2}\left(s_{1}\right)\left[\Psi_{n, 1}^{2}\left(s_{1}\right) \Psi_{n, 1}^{3}\left(s_{1}\right) \nabla v^{1}\left(s_{1}\right)-\Psi_{n, 2}^{2}\left(s_{1}\right) \Psi_{n, 2}^{3}\left(s_{1}\right) \nabla v^{2}\left(s_{1}\right)\right]\right|_{L^{2}} d s_{1}\right)^{2}\right] \\
\leq & C\left(\frac{3-2 \delta}{1-2 \delta}\right)\left(t^{\frac{3-2 \delta}{2}}+t^{\frac{1-2 \delta}{2}}\right) \mathbb{E}\left[\operatorname { s u p } _ { 0 \leq s \leq t } \left(\int_{0}^{s}\left(\Psi_{n, 2}^{1}\left(s_{1}\right)\right)^{q}\right.\right. \\
& \left.\left.\times\left|\xi^{2}\left(s_{1}\right)\left[\Psi_{n, 1}^{2}\left(s_{1}\right) \Psi_{n, 1}^{3}\left(s_{1}\right) \nabla v^{1}\left(s_{1}\right)-\Psi_{n, 2}^{2}\left(s_{1}\right) \Psi_{n, 2}^{3}\left(s_{1}\right) \nabla v^{2}\left(s_{1}\right)\right]\right|_{L^{2}}^{q} d s_{1}\right)^{\frac{2}{q}}\right] .
\end{aligned}
$$

For $\mathrm{j}=1,2$, we define the following stopping times

$$
\begin{aligned}
\tau_{j}^{u}:=\inf _{s>0}\left\{\left|\nabla v^{j}(s)\right|_{L^{2}} \geq n^{1-\rho}\right\} \wedge T, & \tau_{j}^{o}:=\inf _{s>0}\left\{\left|\nabla v^{j}(s)\right|_{L^{2}} \geq(2 n)^{1-\rho}\right\} \wedge T \\
\tilde{\tau}_{j}^{u}:=\inf _{s>0}\left\{\int_{0}^{s}\left|\nabla v^{j}\left(s_{1}\right)\right|_{H^{1}}^{2} d s_{1} \geq n^{\rho}\right\} \wedge T, & \tilde{\tau}_{j}^{o}:=\inf _{s>0}\left\{\int_{0}^{s}\left|\nabla v^{j}\left(s_{1}\right)\right|_{H^{1}}^{2} d s_{1} \geq(2 n)^{\rho}\right\} \wedge T, \\
\hat{\tau}_{j}^{u}:=\inf _{s>0}\left\{\int_{0}^{s}\left|\nabla v^{j}\left(s_{1}\right)\right|_{H^{\rho}}^{q} d s_{1} \geq n\right\} \wedge T, & \hat{\tau}_{j}^{o}:=\inf _{s>0}\left\{\int_{0}^{s}\left|\nabla v^{j}\left(s_{1}\right)\right|_{H^{\rho}}^{q} d s_{1} \geq 2 n\right\} \wedge T .
\end{aligned}
$$

From (3.31) it follows that $\hat{\tau}_{j}^{u} \geq \tau_{j}^{u}:=\min \left(\tau_{j}^{u}, \tilde{\tau}_{j}^{u}\right)$ for $q=\frac{4}{1+2 \delta}$. Now, let us assume that $0 \leq s \leq \tau_{j}^{u}$. Then we get by (3.28) and (3.29)

$$
\begin{aligned}
& \left(\int_{0}^{s}\left(\Psi_{n, 2}^{1}\left(s_{1}\right)\right)^{q}\left|\xi^{2}\left(s_{1}\right)\left[\Psi_{n, 1}^{2}\left(s_{1}\right) \Psi_{n, 1}^{3}\left(s_{1}\right) \nabla v^{1}\left(s_{1}\right)-\Psi_{n, 2}^{2}\left(s_{1}\right) \Psi_{n, 2}^{3}\left(s_{1}\right) \nabla v^{2}\left(s_{1}\right)\right]\right|_{L^{2}}^{q} d s_{1}\right)^{2 / q} \\
& \leq C \int_{0}^{s}\left(\left(\Psi_{n, 2}^{1}\left(s_{1}\right)\right)^{q}\left|\xi^{2}\left(s_{1}\right)\right|_{L^{1}}^{q}\left|\nabla v^{1}\left(s_{1}\right)-\nabla v^{2}\left(s_{1}\right)\right|_{H^{\rho}}^{q} d s_{1}\right)^{2 / q} \\
& \leq C(2 n)^{2}\left(\sup _{0 \leq s_{1} \leq s}\left|\nabla v^{1}\left(s_{1}\right)-\nabla v^{2}\left(s_{1}\right)\right|_{L^{2}}^{2(1-\rho)}\right)\left(\int_{0}^{s}\left|\nabla v^{1}\left(s_{1}\right)-\nabla v^{2}\left(s_{1}\right)\right|_{H^{1}}^{2} d s_{1}\right)^{\rho} .
\end{aligned}
$$

The Cauchy-Schwarz inequality and estimate (3.26) give for $0 \leq s \leq \underline{\tau}_{j}^{u}$

$$
\begin{aligned}
& \mathbb{E}\left[\sup _{0 \leq s \leq t}\left(\int_{0}^{s}\left(\Psi_{n, 2}^{1}\left(s_{1}\right)\right)^{q}\left|\xi^{2}\left(s_{1}\right)\left[\Psi_{n, 1}^{2}\left(s_{1}\right) \Psi_{n, 1}^{3}\left(s_{1}\right) \nabla v^{1}\left(s_{1}\right)-\Psi_{n, 2}^{2}\left(s_{1}\right) \Psi_{n, 2}^{3}\left(s_{1}\right) \nabla v^{2}\left(s_{1}\right)\right]\right|_{L^{2}}^{q} d s_{1}\right)^{2 / q}\right] \\
& \leq C(2 n)^{2} t^{2}\left(\mathbb{E}\left[\sup _{0 \leq s \leq t}\left|\xi_{1}(s)-\xi_{2}(s)\right|_{L^{2}}^{2}\right]\right)^{1-\rho}\left(\mathbb{E}\left[\sup _{0 \leq s \leq t}\left|\xi_{1}(s)-\xi_{2}(s)\right|_{L^{2}}^{2}\right]\right)^{\rho} \\
& \leq C(2 n)^{2} t^{2}\left\|\xi^{1}-\xi^{2}\right\|_{\mathcal{M}_{\mathfrak{L}}^{2}\left(0, T ; L^{2}\right)}^{2} .
\end{aligned}
$$

Next, let us assume that $\underline{\tau}_{j}^{u}<s \leq \underline{\tau}_{j}^{o}:=\max \left(\tau_{j}^{o}, \tilde{\tau}_{j}^{o}\right)$. Then we get by (3.28) and (3.29)

$$
\left(\int_{0}^{s}\left(\Psi_{n, 2}^{1}\left(s_{1}\right)\right)^{q}\left|\xi^{2}\left(s_{1}\right)\left[\Psi_{n, 1}^{2}\left(s_{1}\right) \Psi_{n, 1}^{3}\left(s_{1}\right) \nabla v^{1}\left(s_{1}\right)-\Psi_{n, 2}^{2}\left(s_{1}\right) \Psi_{n, 2}^{3}\left(s_{1}\right) \nabla v^{2}\left(s_{1}\right)\right]\right|_{L^{2}}^{q} d s_{1}\right)^{2 / q}
$$




$$
\begin{aligned}
\leq & C(2 n)^{2}\left\{\sup _{0 \leq s_{1} \leq s}\left|\nabla v^{1}\left(s_{1}\right)-\nabla v^{2}\left(s_{1}\right)\right|_{L^{2}}^{2}\left(\int_{0}^{s}\left|\nabla v^{1}\left(s_{1}\right)\right|_{H^{1}}^{q} d s_{1}\right)^{2 / q}\right. \\
& +\sup _{0 \leq r \leq s}\left(\int_{0}^{r}\left|\nabla v^{1}\left(s_{1}\right)-\nabla v^{2}\left(s_{1}\right)\right|_{H^{1}}^{2} d s_{1}\right) \\
& \left.+\sup _{0 \leq s_{1} \leq s}\left|\nabla v^{1}\left(s_{1}\right)-\nabla v^{2}\left(s_{1}\right)\right|_{L^{2}}^{2(1-\rho)}\left(\int_{0}^{s}\left|\nabla v^{1}\left(s_{1}\right)-\nabla v^{2}\left(s_{1}\right)\right|_{H^{1}}^{2} d s_{1}\right)^{2 / q}\right\} .
\end{aligned}
$$

Taking expectation, applying the Cauchy-Schwarz inequality, and taking into account the fact that $\Psi \leq 1$, we infer

$$
\begin{aligned}
& \mathbb{E}\left[\sup _{0 \leq s \leq t}\left(\int_{0}^{s}\left(\Psi_{n, 2}^{1}\left(s_{1}\right)\right)^{q}\left|\xi^{2}\left(s_{1}\right)\left[\Psi_{n, 1}^{2}\left(s_{1}\right) \Psi_{n, 1}^{3}\left(s_{1}\right) \nabla v^{1}\left(s_{1}\right)-\Psi_{n, 2}^{2}\left(s_{1}\right) \Psi_{n, 2}^{3}\left(s_{1}\right) \nabla v^{2}\left(s_{1}\right)\right]\right|_{L^{2}}^{q} d s_{1}\right)^{2 / q}\right] \\
& \leq C(2 n)^{2} t^{2}\left\|\xi^{1}-\xi^{2}\right\|_{\mathcal{M}_{\mathfrak{A}}^{2}\left(0, T ; L^{2}\right)}^{2} .
\end{aligned}
$$

Taking $C=C(2 n)^{2} t^{2}$ we achieve

$$
\mathbb{E}\left[\sup _{0 \leq s \leq t}\left|I_{2}(s)\right|_{L^{2}}^{2}\right] \leq C\left\|\xi^{1}-\xi^{2}\right\|_{M_{\mathfrak{A}}^{2}\left(0, T ; L^{2}\right)}^{2}
$$

In similar manner, the term $I_{3}$ can be handled using (3.7). In particular, we get

$$
\mathbb{E}\left[\sup _{0 \leq s \leq t}\left|I_{3}(s)\right|_{L^{2}}^{2}\right] \leq 4 t\left\|\xi^{1}-\xi^{2}\right\|_{M_{\mathfrak{A}}^{2}\left(0, T ; L^{2}\right)}^{2}
$$

Combining (3.36), (3.37) and (3.38) in (3.27) appropriately, one can infer (3.22). This completes the proof of Claim 3.2 .

Proof of Lemma 3.1. Let $R>0$ be such that $\mathfrak{T}_{n}$ maps $\mathcal{C}_{R}$ into itself, as given by Claim 3.1 (a). By Claim 3.1 (b) we infer that there exists an $\alpha>0$ such that for any $t \in(0, T]$ there exists a constant $K=K(t, \alpha)>0$ with

$$
\mathbb{E}\left|\mathfrak{T}_{n} \xi(t)\right|_{H^{\alpha}}^{2} \leq K, \quad \xi \in \mathcal{C}_{R}
$$

and a constant $C>0$ such that

$$
\mathbb{E}\left[\sup _{0<t_{1}<t_{2} \leq T} \frac{\left|\mathfrak{T}_{n} \xi\left(t_{2}\right)-\mathfrak{T}_{n} \xi\left(t_{1}\right)\right|_{L^{2}}}{\left(t_{2}-t_{1}\right)^{\delta}}\right] \leq C, \quad \xi \in \mathcal{C}_{R}
$$

In the sequel we show that there exists a probability space $\mathfrak{A}^{*}$ and two Wiener processes $\mathcal{W}_{1}$ and $\mathcal{W}_{2}$ being cylindrical on $\mathcal{H}_{1}$ and $\mathcal{H}_{2}$ (let us remind that the spaces are given in Assumption 2.3) defined over $\mathfrak{A}^{*}$, and a process $u_{n}^{*}$ such that $\mathfrak{T}_{n}\left(u^{*}\right)=u^{*}$. The proof is done in the steps (a) to (f) below.

Step (a): For each $n \in \mathbb{N}$, let $\left\{\left(u_{n}^{(k)}, v_{n}^{(k)}\right): k \in \mathbb{N}\right\}$ be a recurrence sequence defined by the operators $\mathfrak{T}_{n}$ and $\mathfrak{R}$ via the system (3.14) as follows:

$$
u_{n}^{(0)}(t):=0, t \in[0, T], \quad\left(u_{n}^{(k)}, v_{n}^{(k)}\right):=\left(\mathfrak{T}_{n}\left(u_{n}^{(k-1)}\right), \mathfrak{R}\left(u_{n}^{(k-1)}\right)\right), k \geq 1 .
$$

First, observe that the laws of the sequence $\left\{\left(u_{n}^{(k)}, v_{n}^{(k)}\right): k \in \mathbb{N}\right\}$ is a tight family on $C_{b}^{0}\left([0, T] ; L^{2}(\mathcal{O})\right) \times C_{b}^{0}\left([0, T] ; H^{1}(\mathcal{O})\right)$. In fact, the set of probability measures $\left\{\pi_{j} \circ\left(u_{n}^{(k)}, v_{n}^{(k)}\right)\right.$ : $k \in \mathbb{N}\}$ is tight, due to Claim 3.1. (By $\pi_{j}, j=1,2$, we denote the projection onto the $j^{\text {th }}$ coordinate). To be more precise, by Claim 3.1 (a) we know that $u_{n}^{(k)} \in \mathcal{C}_{R}$, and, by Claim 3.1 (b) we 
know that (3.39) and (3.40) are satisfied. By the Chebyscheff inequality and the Arzela-Ascoli Theorem (see [25, Lemma 2.2, p. 213]), we infer that the family of probability laws induced by $\left\{\pi_{1} \circ\left(u_{n}^{(k)}, v_{n}^{(k)}\right): k \in \mathbb{N}\right\}$ is tight. The tightness of the laws of the sequence $\left\{v_{n}^{(k)}: k \in \mathbb{N}\right\}$ follows by the following observations. Invoking Lemma A.3 with $E=H^{1}(\mathcal{O})$ (see Assumption A.1), $\bar{\alpha}=1, \gamma=-\frac{1}{2}, \delta=0$ and $q>1, \beta>0$ sufficiently large so that $\bar{\alpha}-\frac{1}{q}<\beta$, we obtain for $\xi \in \mathcal{C}_{R}$

$$
\left\|\int_{0}^{\cdot} e^{(\cdot-s)\left(r_{u} A-\gamma I\right)} \xi(s) d s\right\|_{C_{b}^{\beta}\left([0, T] ; H^{1}\right)} \leq\|\xi\|_{L^{q}\left(0, T ; L^{2}\right)} .
$$

Secondly, it follows from Corollary A.7 (see also [34, Lemma 3.3]) setting $E=H^{1}(\mathcal{O}), \delta=0$, $\nu=0$, and $p>2, \beta>0$ such large that $\beta+\frac{1}{p}<\frac{1}{2}$

$$
\mathbb{E}\left[\left\|\int_{0}^{\cdot} e^{(\cdot-s)\left(r_{v} A-\alpha I\right)} v(s) d \mathcal{W}_{2}(s)\right\|_{C_{b}^{\beta}\left([0, T] ; H^{1}\right)}^{2}\right] \leq \mathbb{E}\left[\|v\|_{L^{p}\left(0, T ; H^{1}\right)}^{2}\right] .
$$

Since the marginals of $\left\{\left(u_{n}^{(k)}, v_{n}^{(k)}\right): k \in \mathbb{N}\right\}$ form tight families, $\left\{\left(u_{n}^{(k)}, v_{n}^{(k)}\right): k \in \mathbb{N}\right\}$ forms itself a tight family in $C_{b}^{0}\left([0, T] ; L^{2}(\mathcal{O})\right) \times C_{b}^{0}\left([0, T] ; H^{1}(\mathcal{O})\right)$. Hence, the laws of the sequence $\left\{\left(u_{n}^{(k)}, v_{n}^{(k)}\right): k \in \mathbb{N}\right\}$ are a tight family on $C_{b}^{0}\left([0, T] ; L^{2}(\mathcal{O})\right) \times C_{b}^{0}\left([0, T] ; H^{1}(\mathcal{O})\right)$.

Step (b): Due to the tightness of the laws of the sequence $\left\{\left(u_{n}^{(k)}, v_{n}^{(k)}\right): k \in \mathbb{N}\right\}$, there exists a subsequence, again denoted by $\left\{\left(u_{n}^{(k)}, v_{n}^{(k)}\right): k \in \mathbb{N}\right\}$, and a Borel probability law $\rho_{n}^{*}$ on $C_{b}^{0}\left([0, T] ; L^{2}(\mathcal{O})\right) \times C_{b}^{0}\left([0, T] ; H^{1}(\mathcal{O})\right)$ such that

$$
\operatorname{Law}\left(\left(u_{n}^{(k)}, u_{n}^{(k)}\right)\right) \stackrel{k \rightarrow \infty}{\longrightarrow} \rho_{n}^{*},
$$

weakly. Then, by the Skorokhod Lemma, there exists a probability space $\tilde{\mathfrak{A}}_{n}=\left(\tilde{\Omega}_{n}, \tilde{\mathcal{F}}_{n}, \tilde{\mathbb{P}}_{n}\right)$, a sequence of $C_{b}^{0}\left([0, T] ; L^{2}(\mathcal{O})\right) \times C_{b}^{0}\left([0, T] ; H^{1}(\mathcal{O})\right)$-valued random variables $\left\{\left(\tilde{u}_{n}^{(k)}, \tilde{v}_{n}^{(k)}\right): k \in \mathbb{N}\right\}$ and $\left(\tilde{u}_{n}^{*}, \tilde{v}_{n}^{*}\right)$ such that

$$
\begin{aligned}
& \operatorname{Law}\left(\left(\tilde{u}_{n}^{(k)}, \tilde{v}_{n}^{(k)}\right)\right)=\operatorname{Law}\left(\left(u_{n}^{(k)}, v_{n}^{(k)}\right)\right), \\
& \operatorname{Law}\left(\left(\tilde{u}_{n}^{(k)}, \tilde{v}_{n}^{(k)}\right)\right)=\rho_{n}^{*},
\end{aligned}
$$

and $\tilde{\mathbb{P}}_{n}$-a.s. on $C_{b}^{0}\left([0, T] ; L^{2}(\mathcal{O})\right) \times C_{b}^{0}\left([0, T] ; H^{1}(\mathcal{O})\right)$

$$
\left(\tilde{u}_{n}^{(k)}, \tilde{v}_{n}^{(k)}\right) \longrightarrow\left(\tilde{u}_{n}^{*}, \tilde{v}_{n}^{*}\right) \quad \text { as } \quad k \rightarrow \infty
$$

Step (c): For each $k \in \mathbb{N}$, let $\tilde{M}_{1, n}^{(k)}$ and $\tilde{M}_{2, n}^{(k)}$ be the processes given by

$$
\begin{aligned}
\tilde{M}_{1, n}^{(k)}(t):= & \tilde{u}_{n}^{(k)}(t)-u_{0}+\int_{0}^{t}\left[r_{u} A \tilde{u}_{n}^{(k)}(s)-\chi \psi_{n}\left(h^{2}\left(\tilde{v}_{n}^{(k)}(s), s\right)\right) \psi_{n}\left(h^{1}\left(\tilde{u}_{n}^{(k-1)}(s), s\right)\right)\right. \\
& \left.\times \psi_{n}\left(h^{3}\left(\tilde{v}_{n}^{(k)}(s), s\right)\right) \operatorname{div}\left(\tilde{u}_{n}^{(k-1)}(s) \nabla \tilde{v}_{n}^{(k)}(s)\right)-\gamma \tilde{u}_{n}^{(k)}(s)\right] d s \\
\tilde{M}_{2, n}^{(k)}(t):= & \tilde{v}_{n}^{(k)}(t)-v_{0}+\int_{0}^{t}\left[r_{v} A \tilde{v}_{n}^{(k)}(s)-\alpha \tilde{v}_{n}^{(k)}(s)\right] d s-\beta \int_{0}^{t} \tilde{u}_{n}^{(k-1)}(s) d s,
\end{aligned}
$$


and let

$$
\left(\tilde{\mathcal{G}}_{n}^{(k)}\right)_{t}:=\sigma\left(\left\{\left(\tilde{u}_{n}^{(k)}(s), \tilde{v}_{n}^{(k)}(s), \tilde{u}_{n}^{(k-1)}(s)\right): s \leq t,\right\}\right) .
$$

Then, $\tilde{M}_{1, n}^{(k)}$ and $\tilde{M}_{2, n}^{(k)}$ are local martingales over $\left(\tilde{\Omega}_{n}, \tilde{\mathcal{F}}_{n}, \tilde{\mathbb{P}}_{n}\right)$ with respect to the filtration $\left(\mathcal{G}_{n, t}^{(k)}\right)_{t \in[0, T]}$ and with quadratic variation

$$
\begin{aligned}
& \left\langle\tilde{M}_{1, n}^{(k)}\right\rangle_{t}:=\left\langle\tilde{M}_{1, n}^{(k)}(t), \tilde{M}_{1, n}^{(k)}(t)\right\rangle=\int_{0}^{t} \sum_{j \in \mathbb{Z}}\left|\tilde{u}_{n}^{(k), j}(s) \psi_{j}^{(\delta)}\right|^{2} d s, \quad t \in[0, T], \\
& \left\langle\tilde{M}_{2, n}^{(k)}\right\rangle_{t}:=\left\langle\tilde{M}_{2, n}^{(k)}(t), \tilde{M}_{2, n}^{(k)}(t)\right\rangle=\int_{0}^{t} \sum_{j \in \mathbb{Z}}\left|\tilde{v}_{n}^{(k), j}(s) \psi_{j}^{(\delta)}\right|^{2} d s, \quad t \in[0, T] .
\end{aligned}
$$

The representations (3.46) and (3.47) follow from (3.42) and the fact that $u_{n}^{(k)}=\mathfrak{T}_{n}\left(u_{n}^{(k-1)}\right)$; see (3.41). To show that $\left\langle\tilde{M}_{1, n}^{(k)}\right\rangle$ and $\left\langle\tilde{M}_{2, n}^{(k)}\right\rangle$ are adapted to the filtration $\left(\mathcal{G}_{n, t}^{(k)}\right)_{t \in[0, T]}$ we use the fact that by (3.42) for all measurable mapping $\phi_{1}: C_{b}^{0}\left([0, T] ; L^{2}(\mathcal{O})\right) \rightarrow \mathbb{R}, \phi_{2}: C_{b}^{0}\left([0, T] ; H^{1}(\mathcal{O})\right) \rightarrow$ $\mathbb{R}$, and all $0 \leq s \leq t \leq T$ we have

$$
\begin{aligned}
& \mathbb{E}\left[\left(\tilde{M}_{1, n}^{(k)}(t)-\tilde{M}_{1, n}^{(k)}(s)\right) \phi_{1}\left(1_{[0, s)} \tilde{u}_{n}^{(k)}\right) \phi_{2}\left(1_{[0, s)} \tilde{v}_{n}^{(k)}\right)\right]=0, \\
& \mathbb{E}\left[\left(\tilde{M}_{2, n}^{(k)}(t)-\tilde{M}_{2, n}^{(k)}(s)\right) \phi_{1}\left(1_{[0, s)} \tilde{u}_{n}^{(k)}\right) \phi_{2}\left(1_{[0, s)} \tilde{v}_{n}^{(k)}\right)\right]=0 .
\end{aligned}
$$

In addition, we have for all $w_{1}, w_{2} \in H^{\delta}$ for $\delta>1$,

$$
\begin{aligned}
& \tilde{\mathbb{E}}\left[\left\langle\tilde{M}_{1, n}^{(k)}(t), w_{1}\right\rangle\left\langle\tilde{M}_{1, n}^{(k)}(t), w_{2}\right\rangle\right. \\
& \left.-\left\langle\tilde{M}_{1, n}^{(k)}(s), w_{1}\right\rangle\left\langle\tilde{M}_{1, n}^{(k)}(s), w_{2}\right\rangle-\int_{s}^{t}\left\langle\tilde{u}_{n}^{(k)}(r), w_{1}\right\rangle\left\langle\tilde{u}_{n}^{(k)}(r), w_{2}\right\rangle d r\right]=0, \\
& \tilde{\mathbb{E}}\left[\left\langle\tilde{M}_{2, n}^{(k)}(t), w_{1}\right\rangle\left\langle\tilde{M}_{2, n}^{(k)}(t), w_{2}\right\rangle\right. \\
& \left.-\left\langle\tilde{M}_{2, n}^{(k)}(s), w_{1}\right\rangle\left\langle\tilde{M}_{2, n}^{(k)}(s), w_{2}\right\rangle-\int_{s}^{t}\left\langle\tilde{v}_{n}^{(k)}(r), w_{1}\right\rangle\left\langle\tilde{v}_{n}^{(k)}(r), w_{2}\right\rangle d r\right]=0 .
\end{aligned}
$$

Step (d): Next, we verify the following statements with the aim to pass the limit.

a) There exists a constant $C_{n}>0$ such that $\sup _{k \in \mathbb{N}} \tilde{\mathbb{E}}^{n}\left[\sup _{0 \leq s \leq T}\left|\tilde{u}_{n}^{(k)}(s)\right|_{L^{2}}^{2}\right] \leq C_{n}$ for all $n \in \mathbb{N}$ and

b) for any $r \in(1,2)$ we have

$$
\lim _{k \rightarrow \infty} \tilde{\mathbb{E}}^{n}\left[\sup _{0 \leq s \leq T}\left|\tilde{u}_{n}^{(k)}(s)-\tilde{u}_{n}^{*}\right|_{L^{2}}^{r}\right]=0 .
$$

Let us recall that by our construction which uses the Skorokhod Embedding Theorem, the laws of $u_{n}^{(k)}$ and $\tilde{u}_{n}^{(k)}$ on $C_{b}^{0}\left([0, T] ; L^{2}(\mathcal{O})\right)$ are identical for any $k \in \mathbb{N}$. Hence,

$$
\mathbb{E}\left[\sup _{0 \leq s \leq T}\left|u_{n}^{(k)}(s)\right|_{L^{2}}^{2}\right]=\tilde{\mathbb{E}}^{n}\left[\sup _{0 \leq s \leq T}\left|\tilde{u}_{n}^{(k)}(s)\right|_{L^{2}}^{2}\right]
$$

and part (a) easily follows from the fact that $u_{n}^{(k)} \in \mathcal{M}_{\mathfrak{A}}^{2}\left(0, T ; L^{2}(\mathcal{O})\right)$. It follows from part (a) that for any $r<2$ the process $\sup _{0 \leq s \leq T}\left|\tilde{u}_{n}^{(k)}(s)\right|_{L^{2}}^{r}$ is uniformly integrable with respect to the 
probability measure $\tilde{\mathbb{P}}_{n}$. Since $\tilde{\mathbb{P}}_{n^{-}}$-a.s. $\tilde{u}_{n}^{(k)} \rightarrow u_{n}^{*}$ and $\sup _{0 \leq s \leq T}\left|\tilde{u}_{n}^{(k)}(s)\right|_{L^{2}}^{r}$ is uniformly integrable w.r.t. the probability measure $\tilde{\mathbb{P}}_{n}$, we obtain part (b) thanks to the applicability of the Vitali Convergence Theorem.

Step (e): For any $r<2$, since $\sup _{0 \leq s \leq T}\left|\tilde{u}_{n}^{(k)}(s)\right|_{L^{2}}^{r}$ is uniformly integrable with respect to the probability measure $\tilde{\mathbb{P}}_{n}$, it is integrable for $r=2$. Moreover,

$$
\int_{0}^{t} \sum_{j \in \mathbb{Z}}\left|\tilde{u}_{n}^{(k), j}(s) \psi_{j}^{(\delta)}\right|^{2} d s+\int_{0}^{t} \sum_{j \in \mathbb{Z}}\left|\tilde{v}_{n}^{(k), j}(s) \psi_{j}^{(\delta)}\right|^{2} d s \leq C \int_{0}^{T}\left|\tilde{u}_{n}^{(k)}(s)\right|_{L^{2}}^{2} d s .
$$

Hence we can invoke the Vitali Convergence Theorem to take the limit in (3.44) and (3.45) to define

$$
M_{1, n}^{*}:=\lim _{k \rightarrow \infty} M_{1, n}^{(k)} \quad \text { and } \quad M_{2, n}^{*}:=\lim _{k \rightarrow \infty} M_{2, n}^{(k)}
$$

Next, let $\tilde{\mathbb{G}}^{n}=\left(\tilde{\mathcal{G}}_{t}^{n}\right)_{t \in[0, T]}$ be the filtration defined by

$$
\tilde{\mathcal{G}}_{t}^{n}=\sigma\left(\sigma\left(\left(\tilde{u}_{n}^{*}(s), \tilde{v}_{n}^{*}(s)\right) ; 0 \leq s \leq t\right) \cup \mathcal{N}\right), \quad t \in[0, T]
$$

where $\mathcal{N}$ denotes the set of null sets of $\tilde{\mathcal{F}}^{n}$. Since $u_{n}^{(k)} \in L^{2}\left(0, T ; H^{1}(\mathcal{O})\right)$, the limit is well defined and for $0 \leq s \leq t$

$$
\mathbb{E}\left[\left(\tilde{M}_{1, n}^{*}(t)-\tilde{M}_{1, n}^{*}(s)\right) \phi_{1}\left(1_{[0, s)} \tilde{u}_{n}^{*}\right) \phi_{2}\left(1_{[0, s)} \tilde{v}_{n}^{*}\right)\right]=0,
$$

and

$$
\mathbb{E}\left[\left(\tilde{M}_{2, n}^{*}(t)-\tilde{M}_{2, n}^{*}(s)\right) \phi_{1}\left(1_{[0, s)} \tilde{u}_{n}^{*}\right) \phi_{2}\left(1_{[0, s)} \tilde{v}_{n}^{*}\right)\right]=0 .
$$

In addition, we have for all $w_{1}, w_{2} \in H^{\delta}$ with $\delta>1$,

$$
\begin{aligned}
& \tilde{\mathbb{E}}\left[\left\langle\tilde{M}_{1, n}^{*}(t), w_{1}\right\rangle\left\langle\tilde{M}_{1, n}^{*}(t), w_{2}\right\rangle-\left\langle\tilde{M}_{1, n}^{*}(s), w_{1}\right\rangle\left\langle\tilde{M}_{1, n}^{*}(s), w_{2}\right\rangle-\int_{s}^{t}\left\langle\tilde{u}_{n}^{*}(r), w_{1}\right\rangle\left\langle\tilde{u}_{n}^{*}(r), w_{2}\right\rangle d r\right]=0, \\
& \tilde{\mathbb{E}}\left[\left\langle\tilde{M}_{2, n}^{*}(t), w_{1}\right\rangle\left\langle\tilde{M}_{2, n}^{*}(t), w_{2}\right\rangle-\left\langle\tilde{M}_{2, n}^{*}(s), w_{1}\right\rangle\left\langle\tilde{M}_{2, n}^{*}(s), w_{2}\right\rangle-\int_{s}^{t}\left\langle\tilde{v}_{n}^{*}(r), w_{1}\right\rangle\left\langle\tilde{v}_{n}^{*}(r), w_{2}\right\rangle d r\right]=0 .
\end{aligned}
$$

By the representation theorem [11, Theorem 8.2], there exists a filtered probability space $\left(\tilde{\tilde{\Omega}}_{n}, \tilde{\tilde{\mathcal{F}}}_{n}, \tilde{\widetilde{\mathbb{P}}}_{n}\right)$ with filtration $\left(\tilde{\tilde{\mathcal{F}}}_{t}^{n}\right)_{t \in[0, T]}$, two cylindrical Wiener processes $\tilde{\mathcal{W}}_{1}$ and $\tilde{\mathcal{W}}_{2}$ on $\mathcal{H}_{1}$ and $\mathcal{H}_{2}$ defined on $\mathfrak{A}_{n}^{*}:=\left(\tilde{\Omega}_{n} \times \tilde{\tilde{\Omega}}_{n}, \tilde{\mathcal{F}}_{n} \times \tilde{\mathcal{F}}_{n}, \tilde{\mathbb{P}}_{n} \times \tilde{\mathbb{P}}_{n}\right)$ and adapted to $\left(\tilde{\mathcal{F}}_{t}^{n} \times \tilde{\mathcal{F}}_{t}^{n}\right)_{t \in[0, T]}$ such that

$$
\begin{aligned}
& M_{1}(t, \omega, \tilde{\omega}):=\int_{0}^{t} u_{n}^{*}(s, \tilde{\omega}) d \tilde{\mathcal{W}}_{1}(s, \tilde{\omega}, \tilde{\tilde{\omega}}), \quad t \in[0, T],(\tilde{\omega}, \tilde{\tilde{\omega}}) \in \tilde{\Omega} \times \tilde{\tilde{\Omega}}, \\
& \left.M_{2}(t, \omega, \tilde{\omega}):=\int_{0}^{t} v_{n}^{*}(s, \tilde{\omega})\right) d \tilde{\mathcal{W}}_{2}(s, \tilde{\omega}, \tilde{\tilde{\omega}}), \quad t \in[0, T],(\tilde{\omega}, \tilde{\tilde{\omega}}) \in \tilde{\Omega} \times \tilde{\tilde{\Omega}} .
\end{aligned}
$$

In this way, we obtain a pair $\left(u_{n}^{*}, v_{n}^{*}\right) \in C_{b}^{0}\left([0, t] ; L^{2}(\mathcal{O})\right) \times C_{b}^{0}\left([0, t] ; H^{1}(\mathcal{O})\right)$ over $\mathfrak{A}^{*}$ and two cylindrical Wiener processes $\mathcal{W}_{1}$ and $\mathcal{W}_{2}$ on $H^{\delta}$ for $\delta>1$ over $\mathfrak{A}^{*}$. 
Step (f): In the next step we show that $\left(u_{n}^{*}, v_{n}^{*}\right)$ over $\mathfrak{A}^{*}$ together with $\mathcal{W}_{1}$ and $\mathcal{W}_{2}$ is indeed a martingale solution to (3.13). Let us consider the Banach space $\mathcal{M}_{\mathfrak{A}_{n}^{*}}^{2}\left(0, T ; L^{2}(\mathcal{O})\right)$ over $\mathfrak{A}_{n}^{*}$ and the convex subset $\tilde{\mathcal{C}}_{R}^{n, *}$. The operator $\tilde{\mathfrak{T}}_{n}$ acts now on the space $\tilde{\mathcal{C}}_{R}^{n}$ and is defined by system (3.14).

Now, we will show in this step that for any $\varepsilon>0$ we have (here we denote the expectation over $\mathfrak{A}_{n}^{*}$ by $\left.\mathbb{E}_{n}^{*}\right)$

$$
\mathbb{E}_{n}^{*}\left[\sup _{0 \leq s \leq T}\left|\tilde{\mathfrak{T}}_{n}\left(\tilde{u}_{n}^{*}\right)(s)-\tilde{u}_{n}^{*}(s)\right|_{L^{2}}\right] \leq \varepsilon
$$

Fix $\varepsilon>0$. We can write for all $n \in \mathbb{N}, n>1$,

$$
\tilde{\mathfrak{T}}_{n}\left(\tilde{u}_{n}^{*}\right)-\tilde{u}_{n}^{*}=\tilde{u}_{n}^{*}-\tilde{u}_{n}^{(k)}+\tilde{u}_{n}^{(k)}-\tilde{\mathfrak{T}}_{n}\left(\tilde{u}_{n}^{(k-1)}\right)+\tilde{\mathfrak{T}}_{n}\left(u_{n}^{(k-1)}\right)-\tilde{\mathfrak{T}}_{n}\left(\tilde{u}_{n}^{*}\right) .
$$

Note, since $\left\{\tilde{u}_{n}^{(k)}: k \in \mathbb{N}\right\}$ converges to $\tilde{u}_{n}^{*} \tilde{\mathbb{P}}$-a.s. there exists a number $K_{0}$ such that

$$
\mathbb{E}_{n}^{*}\left[\sup _{0 \leq s \leq T}\left|\tilde{u}_{n}^{*}(s)-\tilde{u}_{n}^{(k)}(s)\right|_{L^{2}}^{r}\right] \leq \frac{\varepsilon}{3}, \quad \forall k \geq K_{0}
$$

Secondly, we know by (3.42), (3.43), and the construction of the sequence $\left\{\tilde{u}_{n}^{(k)}: k \in \mathbb{N}\right\}$ that we have for all $k \in \mathbb{N}$

$$
\left(3.50 \mathbb{F}_{n}^{*}\left[\sup _{0 \leq s \leq T}\left|\tilde{u}_{n}^{(k)}(s)-\tilde{\mathfrak{T}}_{n}\left(\tilde{u}_{n}^{(k-1)}\right)(s)\right|_{L^{2}}^{m}\right]=\mathbb{E}_{n}^{*}\left[\sup _{0 \leq s \leq T}\left|u_{n}^{(k)}(s)-\mathfrak{T}_{n}\left(u_{n}^{(k-1)}\right)(s)\right|_{L^{2}}^{m}\right]=0 .\right.
$$

Let us recall that we have shown that $\mathfrak{T}_{n}$ is continuous on $\mathcal{M}_{\mathfrak{A}}^{2}\left(0, T ; L^{2}(\mathcal{O})\right)$. Hence, there exists a $\delta>0$ such that for all $\xi, \eta \in \mathcal{M}_{\mathfrak{A}^{*}}^{2}\left(0, T ; L^{2}(\mathcal{O})\right)$ with $\|\xi-\eta\|_{\mathcal{M}_{\mathfrak{A}^{*}}^{2}\left(0, T ; L^{2}\right)} \leq \delta$, we have

$$
\left\|\mathfrak{T}_{n}(\xi)-\mathfrak{T}_{n}(\eta)\right\|_{\mathcal{M}_{\mathfrak{A}^{*}}^{2}\left(0, T ; L^{2}\right)}^{r} \leq \frac{\varepsilon}{3}
$$

In addition, it follows from the identity $\operatorname{Law}\left(\tilde{u}_{n}^{(k)}\right)=\operatorname{Law}\left(u_{n}^{(k)}\right)$, the property $\tilde{u}_{n}^{(k)} \in \mathcal{M}_{\tilde{\mathfrak{A}}_{n}}^{2}\left(0, T ; L^{2}(\mathcal{O})\right)$ for all $k \in \mathbb{N}$, and the convergence of $\left\{\tilde{u}_{k}^{(k)}: k \in \mathbb{N}\right\}$ to $\tilde{u}_{n}^{*}$ that there exists a number $N_{1}>0$ such that

$$
\mathbb{E}_{n}^{*}\left[\sup _{0 \leq s \leq T}\left|\tilde{u}_{n}^{(k-1)}(s)-\mathfrak{T}_{n}\left(\tilde{u}_{n}^{*}\right)(s)\right|_{L^{2}}\right] \leq \delta,
$$

for all $k \geq K_{1}$. In particular, applying the triangle inequality to (3.48), then the estimate (3.49), the identity (3.50), and the estimate (3.51) together with (3.52) gives that for all $k \geq$ $\max \left(K_{0}, K_{1}\right)$ we have

$$
\mathbb{E}_{n}^{*}\left[\sup _{0 \leq s \leq T}\left|\tilde{\mathfrak{T}}_{n} \tilde{u}_{n}^{*}(s)-\tilde{u}_{n}^{*}(s)\right|_{L^{2}}\right] \leq \varepsilon
$$

Hence, for all $t \in[0, T]$ we have $\mathbb{P}$-a.s.

$$
\tilde{\mathfrak{T}}_{n}\left(\tilde{u}_{n}^{*}\right)(t)=\tilde{u}_{n}^{*}(t),
$$

which is the assertion. This completes the proof of Lemma 3.1 .

Step II: In this step we will show that the solution $\left(u_{n}, v_{n}\right)$ is non-negative. For being short, we omit tedious steps and give a brief outline of the proof. For more details, one can follow e.g. Theorem 2.3 in [32], or Section 2.6 of [2]. 
Claim 3.3. For any $n \in \mathbb{N}$ the solutions $u_{n}$ and $v_{n}$ to the system (3.13) are non-negative.

To prove Claim 3.3, we follow the steps involved in the proof of Theorem 2.6.2 of [2]. For being short and precise, we provide below a brief sketch of the proof.

Proof. For any $x \in L^{2}$ we set

$$
x^{+}:=\max \{x, 0\}, \quad x^{-}:=\max \{-x, 0\} .
$$

Let us define for $\delta \in(0,1)$,

$$
g_{\delta}(r):=\frac{r^{2}}{\delta+r}, \quad r \in(-\delta, \infty), \quad \text { and } \quad G_{\delta}(r):=g_{\delta}\left(\left(r^{-}\right)^{2}\right), \quad r \in \mathbb{R} .
$$

Then, $G_{\delta}$ belongs to $C^{2}$ and $G_{\delta}(r)=G_{\delta}^{\prime}(r)=G_{\delta}^{\prime \prime}(r)=0$ for all $r \in[0, \infty)$, and $\left|G_{\delta}^{\prime}(r)\right| \leq 2 r^{-}$, and $\left|G_{\delta}^{\prime \prime}(r)\right| \leq 8$ for all $r \in \mathbb{R}$. Now, let us define $\phi_{\delta}: L^{2}(\mathcal{O}) \rightarrow \mathbb{R}$ by

$$
\phi_{\delta}\left(u_{n}\right)=\int_{\mathcal{O}} G_{\delta}\left(u_{n}(x)\right) d x, \quad u_{n} \in L^{2}(\mathcal{O}) .
$$

Then $\phi_{\delta}$ is twice Gateau differentiable on $L^{2}(\mathcal{O})$. We now apply Itô formula to $\phi_{\delta}\left(u_{n}(t)\right)$. However, to be precise, one should replace the Laplace operator $\Delta$ by its Yosida approximation $\frac{1}{\varepsilon}\left(i d-(\varepsilon A-i d)^{-1}\right)$, apply then the Itô formula to $\phi_{\delta}\left(u_{n}(t)\right)$, where $\left(u_{n}^{\varepsilon}, v_{n}^{\varepsilon}\right)$ is the solution to system (3.13) in which the Laplace operator is replaced by its Yosida approximation, and then taking the limit $\varepsilon \rightarrow 0$. For convenience we omit the step and apply the the Itô formula directly to $\phi_{\delta}\left(u_{n}(t)\right)$ and get

$$
\begin{aligned}
\mathbb{E}\left[\phi_{\delta}\left(u_{n}(t)\right)\right]= & \phi_{\delta}\left(u_{n}(0)\right)+\mathbb{E}\left[\int_{0}^{t}\left\langle D \phi_{\delta}\left(u_{n}(s)\right), r_{u} A u_{n}(s)-\chi \operatorname{div}\left(u_{n}(s) \nabla v_{n}(s)\right)+\gamma u_{n}(s)\right\rangle d s\right] \\
& +\frac{1}{2} \sum_{k \in \mathbb{Z}} \mathbb{E}\left[\int_{0}^{t} \int_{\mathcal{O}} G_{\delta}^{\prime \prime}\left(u_{n}(s, x)\right)\left[u_{n}(s, x) \psi_{k}^{\left(\delta_{1}\right)}(x)\right]^{2} d x d s\right] .
\end{aligned}
$$

As $u_{0} \in L_{+}^{2}(\mathcal{O})$, i.e., $u_{0} \in L^{2}(\mathcal{O}), u_{0} \geq 0$ a.e. So $\phi_{\delta}\left(u_{n}(0)\right)=0$. Now using Neumann boundary conditions on $\left(u_{n}, v_{n}\right), G_{\delta}^{\prime \prime}(r)=0, r \geq 0$, and integration by parts we obtain

$$
\mathbb{E}\left[\phi_{\delta}\left(u_{n}(t)\right)\right] \leq C\left(r_{u}, \chi\right) \mathbb{E}\left[\int_{0}^{t} \int_{\mathcal{O}}\left|u_{n}^{-}(s, x)\right|^{2}\left|\nabla v_{n}(s, x)\right|^{2} d x d s\right]+(2 \gamma+4) \mathbb{E}\left[\int_{0}^{t}\left|u_{n}^{-}(s)\right|_{L^{2}}^{2} d s\right] .
$$

Letting $\delta \rightarrow 0$ and using Dominated Convergence Theorem, we have

$$
\mathbb{E}\left[\left|u_{n}^{-}(t)\right|_{L^{2}}^{2}\right] \leq C\left(r_{u}, \chi\right) \mathbb{E}\left[\int_{0}^{t} \int_{\mathcal{O}}\left|u_{n}^{-}(s, x)\right|^{2}\left|\nabla v_{n}(s, x)\right|^{2} d x d s\right]+(2 \gamma+4) \mathbb{E}\left[\int_{0}^{t}\left|u_{n}^{-}(s)\right|_{L^{2}}^{2} d s\right] .
$$

Using Grownwall's Lemma we have $\mathbb{E}\left[\left|u_{n}^{-}(t)\right|_{L^{2}}^{2}\right]=0$ and this implies

$$
u_{n}^{-}(t)=0 \quad \text { a.e. } \quad t \in[0, T], x \in \mathcal{O}, \omega \in \Omega .
$$

Omitting $u_{n}$, then the positivity of $v_{n}$ follows again by the same arguments as before (or used by Tessitore and Zabczyk [32]); the term $u_{n}$ can be added by comparison principle (see Kotelenez [23]). Another possibility is to follow [2, Section 2.6]. This completes the proof of Claim 3.3 . 
Step III: Here, we will construct a family of solutions $\left\{\left(\bar{u}_{n}, \bar{v}_{n}\right): n \in \mathbb{N}\right\}$ following the solution to the original problem until a stopping time $\bar{\tau}_{n}$. In particular, we will introduce for each $n \in \mathbb{N}$ a new pair of processes $\left(\bar{u}_{n}, \bar{v}_{n}\right)$ following the chemotaxis system up to the stopping time $\bar{\tau}_{n}$. Besides, we will have $\left.\left(\bar{u}_{n}, \bar{v}_{n}\right)\right|_{\left[0, \bar{\tau}_{n}\right)}=\left.\left(\bar{u}_{n+1}, \bar{v}_{n+1}\right)\right|_{\left[0, \bar{\tau}_{n}\right)}$.

Let us start with $n=1$. From Lemma 3.1, we know there exists a martingale solution consisting of a probability space $\mathfrak{A}_{1}=\left(\Omega_{1}, \mathcal{F}_{1} ;\left(\mathcal{F}_{t}^{1}\right)_{t \in[0, T]}, \mathbb{P}_{1}\right)$, two independent Wiener processes $\left(\mathcal{W}_{1}^{1}, \mathcal{W}_{2}^{1}\right)$ defined over $\mathfrak{A}_{1}$, and a couple of processes $\left(u_{1}, v_{1}\right)$ solving $\mathbb{P}_{1}$-a.s. the system

$$
\left\{\begin{aligned}
& d u_{1}(t)-\left[r_{u} A u_{1}(t)-\chi \psi_{1}\left(h^{1}\left(u_{1}, t\right)\right) \psi_{1}\left(h^{2}\left(v_{1}, t\right)\right) \psi_{1}\left(h^{3}\left(v_{1}, t\right)\right)\right. \\
&\left.\times \operatorname{div}\left(u_{1}(t) \nabla v_{1}(t)\right)+\gamma u_{1}(t)\right] d t=u_{1}(t) d \mathcal{W}_{1}^{1}(t), \\
& d v_{1}(t)-\left[r_{v} A v_{1}(t)-\alpha v(t)\right] d t=\beta u_{1}(t) d t+v_{1}(t) d \mathcal{W}_{2}^{1}(t), \\
&\left(u_{1}(0), v_{1}(0)\right)=\left(u_{0}, v_{0}\right) .
\end{aligned}\right.
$$

Let us define now the stopping times

$$
\begin{aligned}
\tau_{1}^{1} & :=\inf \left\{s \geq 0:\left|u_{1}(s)\right|_{L^{1}} \geq 1\right\} \\
\tau_{1}^{2} & :=\inf \left\{s \geq 0:\left|\nabla v_{1}(s)\right|_{L^{2}}^{2} \geq 1\right\} \\
\tau_{1}^{3} & :=\inf \left\{s \geq 0: \int_{0}^{s}\left|\nabla v_{1}(r)\right|_{H^{1}}^{2} d r \geq 1\right\} .
\end{aligned}
$$

Put $\tau_{1}^{*}:=\min \left(\tau_{1}^{1}, \tau_{1}^{2}, \tau_{1}^{3}\right)$. Observe, on the time interval $\left[0, \tau_{1}^{*}\right)$, the pair $\left(u_{1}, v_{1}\right)$ solves the Chemotaxis system given in (3.11). Now, we define a new pair of processes $\left(\bar{u}_{1}, \bar{v}_{1}\right)$ following $\left(u_{1}, v_{1}\right)$ on $\left[0, \tau_{1}^{*}\right)$ and extend this processes to the whole interval $[0, T]$ in the following way. First, we put $\overline{\mathfrak{A}}_{1}:=\mathfrak{A}_{1}$ and $\overline{\mathcal{W}}_{1}^{j}:=\mathcal{W}_{1}^{j}, j=1,2$, let us introduce the processes $y_{1}$ and $y_{2}$ being a strong solution over $\overline{\mathfrak{A}}_{1}$ to

$$
y_{1}\left(t, u_{1}\left(\tau_{1}^{*}\right), \sigma\right)=e^{t\left(r_{u} A+\gamma I\right)} u_{1}\left(\tau_{1}^{*}\right)+\int_{0}^{t} e^{\left(r_{u} A+\gamma I\right)(t-s)} y_{1}\left(s, u_{1}\left(\tau_{1}^{*}\right), \sigma\right) d\left(\theta_{\sigma} \overline{\mathcal{W}}_{1}^{1}\right)(s)
$$

and

$$
y_{2}\left(t, v_{1}\left(\tau_{1}^{*}\right), \sigma\right)=e^{\left(r_{v} A-\alpha I\right) t} v_{1}\left(\tau_{1}^{*}\right)+\int_{0}^{t} e^{\left(r_{v} A-\alpha I\right)(t-s)} y_{2}\left(s, v_{1}\left(\tau_{1}^{*}\right), \sigma\right) d\left(\theta_{\sigma} \overline{\mathcal{W}}_{2}^{1}\right)(s)
$$

for $t \geq 0$. Here $\theta_{\sigma}$ is the shift operator which maps $\mathcal{W}_{j}(t)$ to $\mathcal{W}_{j}(t+\sigma)$. Since the couple $\left(u_{1}, v_{1}\right)$ is continuous in $\left(L^{2}(\mathcal{O}), H^{1}(\mathcal{O})\right)$, we know that $u_{1}\left(\tau_{1}^{*}\right)$ and $v_{1}\left(\tau_{1}^{*}\right)$ are well defined random variables belonging $\mathbb{P}$-a.s. to $L^{2}(\mathcal{O})$ and $H^{1}(\mathcal{O})$, respectively. Since, in addition, $\left(e^{t\left(r_{u} A+\gamma I\right)}\right)_{t \geq 0}$ and $\left(e^{t\left(r_{v} A-\alpha I\right)}\right)_{t \geq 0}$ are analytic semigroups on $L^{2}(\mathcal{O})$ and $H^{1}(\mathcal{O})$, the existence of a unique solution $y_{j} ; j=1,2$ over $\overline{\mathfrak{A}}_{1}$ to (3.53) and (3.54) in $L^{2}(\mathcal{O})$ and $H^{1}(\mathcal{O})$, respectively, can be shown by standard methods. Now, let us define two processes $\bar{u}_{1}$ and $\bar{v}_{1}$ being identical to $u_{1}$ and $v_{1}$, respectively, on the time interval $\left[0, \tau_{1}^{*}\right)$ and following the heat equation (with lower order terms) with noise, i.e., $y_{1}\left(\cdot, u_{1}\left(\tau_{1}^{*}\right), \tau_{1}^{*}\right)$ and $y_{2}\left(\cdot, v_{1}\left(\tau_{1}^{*}\right), \tau_{1}^{*}\right)$, afterwards. In particular, let

$$
\bar{u}_{1}(t)= \begin{cases}u_{1}(t) & \text { for } 0 \leq t<\tau_{1}^{*}, \\ y_{1}\left(t, u_{1}\left(\tau_{1}^{*}\right), \tau_{1}^{*}\right) & \text { for } \tau_{1}^{*} \leq t \leq T\end{cases}
$$


and

$$
\bar{v}_{1}(t)= \begin{cases}v_{1}(t) & \text { for } 0 \leq t<\tau_{1}^{*}, \\ y_{2}\left(t, v_{1}\left(\tau_{1}^{*}\right), \tau_{1}^{*}\right) & \text { for } \tau_{1}^{*} \leq t \leq T .\end{cases}
$$

Let us now construct the probability space and the processes for the next time interval. First, let $\left(u_{1}\left(\tau_{1}^{*}\right), v_{1}\left(\tau_{1}^{*}\right)\right)$ have probability measure $\mu_{1}$ on $L^{2}(\mathcal{O}) \times H^{1}(\mathcal{O})$. Again, from Lemma 3.1 we know there is a martingale solution consisting of a probability space $\mathfrak{A}_{2}=\left(\Omega_{2}, \mathcal{F}_{2},\left(\mathcal{F}_{t}^{2}\right)_{t \in[0, T]}, \mathbb{P}_{2}\right)$, two independent Wiener processes $\left(\mathcal{W}_{2}^{1}, \mathcal{W}_{2}^{2}\right)$, a couple of processes $\left(u_{2}, v_{2}\right)$ solving $\mathbb{P}_{2}-$ a.s. the system

$$
\left\{\begin{aligned}
d u_{2}(t) & -\left[r_{u} A u_{2}(t)-\chi \psi_{2}\left(h^{1}\left(u_{2}, t\right)\right) \psi_{2}\left(h^{2}\left(v_{2}, t\right)\right) \psi_{2}\left(h^{3}\left(v_{2}, t\right)\right)\right. \\
& \left.\times \operatorname{div}\left(u_{2}(t) \nabla v_{2}(t)\right)+\gamma u_{2}(t)\right] d t=u_{2}(t) d \mathcal{W}_{1}^{2}(t) \\
d v_{2}(t) & -\left[r_{v} A v_{2}(t)-\alpha v(t)\right] d t=\beta u_{2}(t) d t+v_{2}(t) d \mathcal{W}_{2}^{2}(t)
\end{aligned}\right.
$$

with initial condition $\left(u_{2}(0), v_{2}(0)\right)$ having law $\mu_{1}$. Let us define now the stopping times on $\mathfrak{A}_{2}$

$$
\begin{aligned}
\tau_{2}^{1} & :=\inf \left\{s \geq 0:\left|u_{2}(s)\right|_{L^{1}} \geq 2\right\} \\
\tau_{2}^{2} & :=\inf \left\{s \geq 0:\left|\nabla v_{2}(s)\right|_{L^{2}}^{2} \geq 2\right\} \\
\tau_{2}^{3} & :=\inf \left\{s \geq 0: \int_{0}^{s}\left|\nabla v_{2}(r)\right|_{H^{1}}^{2} d r \geq 2\right\}
\end{aligned}
$$

and $\tau_{2}^{*}:=\min \left(\tau_{2}^{1}, \tau_{2}^{2}, \tau_{2}^{3}\right)$. Let $\overline{\mathfrak{A}}_{2}:=\overline{\mathfrak{A}}_{1} \times \mathfrak{A}_{2}, \overline{\mathcal{F}}_{2}:=\overline{\mathcal{F}}_{1} \vee \mathcal{F}_{2}$,

$$
\overline{\mathcal{F}}_{t}^{2}:= \begin{cases}\overline{\mathcal{F}}_{t}^{1}, & \text { if } t<\tau_{1}^{*}, \\ \mathcal{F}_{t-\tau_{1}^{*}}^{2}, & \text { if } t \geq \tau_{1}^{*} .\end{cases}
$$

Finally, let us put for $j=1,2$

$$
\overline{\mathcal{W}}_{2}^{j}(t):= \begin{cases}\overline{\mathcal{W}}_{1}^{j}(t), & \text { if } t<\tau_{1}^{*}, \\ \mathcal{W}_{2}^{j}\left(t-\tau_{1}^{*}\right)+\overline{\mathcal{W}}_{1}^{j}\left(\tau_{1}^{*}\right), & \text { if } t \geq \tau_{1}^{*} .\end{cases}
$$

Now, let us define two processes $\bar{u}_{2}$ and $\bar{v}_{2}$ being identical to $\bar{u}_{1}$ and $\bar{v}_{1}$, respectively, on the time interval $\left[0, \tau_{1}^{*}\right)$, being identical to $u_{2}$ and $v_{2}$ on the time interval $\left[\tau_{1}^{*}, \tau_{1}^{*}+\tau_{2}^{*}\right)$ and following the heat equation (with lower order terms) with multiplicative noise afterwards. Let us note, for any initial condition having distribution equal to $u_{2}\left(\tau_{2}^{*}\right)$ and $v_{2}\left(\tau_{2}^{*}\right)$ that there exists a strong solutions $y_{1}\left(\cdot, \cdot, \tau_{2}^{*}+\tau_{1}^{*}\right)$ and $y_{2}\left(\cdot, \cdot, \tau_{2}^{*}+\tau_{1}^{*}\right)$ of the systems (3.53) and (3.54), respectively, on $\overline{\mathfrak{A}_{2}}$. Let for $t \in[0, T]$

$$
\begin{aligned}
& \bar{u}_{2}(t)= \begin{cases}\bar{u}_{1}(t) & \text { for } 0 \leq t<\tau_{1}^{*}, \\
u_{2}\left(t-\tau_{1}^{*}\right) & \text { for } \tau_{1}^{*} \leq t \leq \tau_{1}^{*}+\tau_{2}^{*}, \\
y_{1}\left(t-\left(\tau_{1}^{*}+\tau_{2}^{*}\right), u_{2}\left(\tau_{2}^{*}\right), \tau_{1}^{*}+\tau_{2}^{*}\right) & \text { for } \tau_{2}^{*}+\tau_{1}^{*} \leq t \leq T,\end{cases} \\
& \bar{v}_{2}(t)= \begin{cases}v_{1}(t) & \text { for } 0 \leq t<\tau_{1}^{*}, \\
v_{2}\left(t-\tau_{1}^{*}\right) & \text { for } \tau_{1}^{*} \leq t \leq \tau_{1}^{*}+\tau_{2}^{*}, \\
y_{2}\left(t-\left(\tau_{1}^{*}+\tau_{2}^{*}\right), v_{2}\left(\tau_{2}^{*}\right), \tau_{1}^{*}+\tau_{2}^{*}\right) & \text { for } \tau_{1}^{*}+\tau_{2}^{*} \leq t \leq T\end{cases}
\end{aligned}
$$


In the same way we will construct for any $n \in \mathbb{N}$ a probability space $\overline{\mathfrak{A}}_{n}$, a pair of Wiener processes $\left(\overline{\mathcal{W}}_{n}^{1}, \overline{\mathcal{W}}_{n}^{2}\right)$, over $\overline{\mathfrak{A}}_{n}$ and a pair of processes $\left(\bar{u}_{n}, \bar{v}_{n}\right)$ starting at $\left(u_{0}, v_{0}\right)$ and solving system (3.11) up to time

$$
\bar{\tau}_{n}:=\tau_{1}^{*}+\cdots+\tau_{n}^{*}
$$

and following the heat equation afterwards. Besides, $\left.\left(\bar{u}_{n}, \bar{v}_{n}\right)\right|_{\left[0, \bar{\tau}_{n-1}\right]}=\left.\left(\bar{u}_{n-1}, \bar{v}_{n-1}\right)\right|_{\left[0, \bar{\tau}_{n-1}\right]}$.

Step IV: In this step, we will give uniform bounds on the processes $\left\{\left(\bar{u}_{n}, \bar{v}_{n}\right): n \in \mathbb{N}\right\}$ by the Lyapunov functional given by

$W(u, v)=\int_{\mathcal{O}}(u(x) \log u(x)-\rho u(x) v(x)) d x+C_{1}|\nabla v|_{L^{2}}^{2}+C_{2}|v|_{L^{2}}^{2}, \quad u \in \operatorname{LlogL}(\mathcal{O}), v \in H^{1}(\mathcal{O})$.

In this way we can show the following claim.

Claim 3.4. There exists a constant $C>0$, such that we have for all $n \in \mathbb{N}$

$$
\mathbb{E}\left[\sup _{0 \leq s \leq T}\left|\bar{u}_{n}(s)\right|_{L^{1}}^{2}\right] \leq C, \quad \mathbb{E}\left[\sup _{0 \leq s \leq T}\left|\nabla \bar{v}_{n}(s)\right|_{L^{2}}^{2}\right] \leq C, \quad \mathbb{E}\left[\int_{0}^{T}\left|\nabla \bar{v}_{n}(s)\right|_{H^{1}}^{2} d s\right] \leq C .
$$

Proof of Claim [3.4. On the time interval $\left[0, \bar{\tau}_{n}\right]$ we use the Lyapunov functional $W\left(\bar{u}_{n}, \bar{v}_{n}\right)$ (where $\bar{\tau}_{n}$ is defined in (3.55) ) and on $\left[\bar{\tau}_{n}, T\right]$ we will use the functional given by

$$
E(u, v)=\int_{\mathcal{O}} u(x) \log u(x) d x+C_{1}|\nabla v|_{L^{2}}^{2}+C_{2}|v|_{L^{2}}^{2}, \quad u \in \operatorname{Llog} \mathrm{L}(\mathcal{O}), v \in H^{1}(\mathcal{O}) .
$$

We note that $\mathbb{E}\left[\sup _{\bar{\tau}_{n} \leq s \leq T} \int_{\mathcal{O}} \bar{u}_{n}(s) \log \bar{u}_{n}(s)\right]$ is bounded below. Hence

$$
\mathbb{E}\left[\sup _{0 \leq s \leq T} 1_{\left[\bar{\tau}_{n}, T\right]}(s) E\left(\bar{u}_{n}(s), \bar{v}_{n}(s)\right)\right]
$$

is bounded below. To show $W\left(\bar{u}_{n}(s), \bar{v}_{n}(s)\right)$ is bounded below on $\left[0, \bar{\tau}_{n}\right]$, it suffices to prove

$$
\mathbb{E}\left[\sup _{0 \leq s \leq \bar{\tau}_{n}} \int_{\mathcal{O}} \bar{u}_{n}(s) \bar{v}_{n}(s)\right]
$$

is bounded below. First, note that, due to the Young inequality, for any $\varepsilon>0$ there exists $C(\varepsilon)>0$ such that we have

$$
\begin{aligned}
\mathbb{E}\left[\sup _{0 \leq s \leq \bar{\tau}_{n}} \int_{\mathcal{O}} \bar{u}_{m}(s, x) \bar{v}_{n}(s, x) d x\right] & \leq \mathbb{E}\left[\sup _{0 \leq s \leq \bar{\tau}_{n}}\left|\bar{u}_{m}(s)\right|_{L^{1}}\left|\bar{v}_{n}(s)\right|_{L^{\infty}}\right] \\
& \leq C(\varepsilon) \mathbb{E}\left[\sup _{0 \leq s \leq \bar{\tau}_{n}}\left|\bar{u}_{m}(s)\right|_{L^{1}}^{2}\right]+\varepsilon \mathbb{E}\left[\sup _{0 \leq s \leq \bar{\tau}_{n}}\left|\bar{v}_{n}(s)\right|_{H^{1}}^{2}\right] .
\end{aligned}
$$

Taking $\varepsilon$ small enough, $\mathbb{E}\left[\sup _{0 \leq s \leq \bar{\tau}_{n}}\left|\bar{v}_{n}(s)\right|_{H^{1}}^{2}\right]$ can be handled by $\mathbb{E}\left[\sup _{0 \leq s \leq \bar{\tau}_{n}}\left(C_{1}|\nabla v|_{L^{2}}^{2}+\right.\right.$ $\left.\left.C_{2}|v|_{L^{2}}^{2}\right)\right]$. To show the functional $W$ is bounded below, we need to show that there exists a constant $C>0$ such that

$$
\mathbb{E}\left[\sup _{0 \leq s \leq \bar{\tau}_{n}}\left|\bar{u}_{m}(s)\right|_{L^{1}}^{2}\right] \leq C .
$$

It remains to show that there exists a constant $C>0$ such that

$$
\left(3 \mathbb{E}\left[{ }_{0} \sup _{0 \leq s \leq T} 1_{\left[0, \bar{\tau}_{n}\right)}(s) W\left(\bar{u}_{m}(s), \bar{v}_{n}(s)\right)\right] \leq C \quad \text { and } \quad \mathbb{E}\left[\sup _{0 \leq s \leq T} 1_{\left[\bar{\tau}_{n}, T\right]}(s) E\left(\bar{u}_{m}(s), \bar{v}_{n}(s)\right)\right] \leq C .\right.
$$


Estimate (3.57) essentially implies (3.56) and

$$
\mathbb{E}\left[\sup _{0 \leq s \leq T}\left|\nabla \bar{v}_{n}(s)\right|_{L^{2}}^{2}\right] \leq C, \quad \mathbb{E}\left[\int_{0}^{T}\left|\nabla \bar{v}_{n}(s)\right|_{H^{1}}^{2} d s\right] \leq C .
$$

Claim 3.5. There exists a constant $C>0$ such that we have for any $n \in \mathbb{N}$

$$
\begin{aligned}
& \mathbb{E}\left[\sup _{0 \leq s \leq t \wedge \bar{\tau}_{n}}\left(W\left(\bar{u}_{n}(s), \bar{v}_{n}(s)\right)-W\left(\bar{u}_{n}(0), \bar{v}_{n}(0)\right)\right)\right] \\
& \quad+\mathbb{E} \int_{0}^{t \wedge \bar{\tau}_{n}}\left(4 r_{u}\left|\nabla \sqrt{\bar{u}_{n}(s)}\right|_{L^{2}}^{2}+\left(C_{1} r_{v}-\left(\chi+\rho r_{u}+\rho r_{v}+C_{1} \beta\right) / \rho \beta\right)\left|A \bar{v}_{n}(s)\right|_{L^{2}}^{2}\right. \\
& \left.\quad+\left(C_{2} r_{v}+C_{1} \alpha\right)\left|\nabla \bar{v}_{n}(s)\right|_{L^{2}}^{2}+C_{2} \alpha\left|\bar{v}_{n}(s)\right|_{L^{2}}^{2}+\frac{1}{2} \rho \beta\left|\bar{u}_{n}(s)\right|_{L^{2}}^{2}+\chi \rho\left\langle\bar{u}_{n}(s),\left(\nabla \bar{v}_{n}(s)\right)^{2}\right\rangle\right) d s \\
& \leq C\left\{t+\mathbb{E} W\left(\bar{u}_{n}(0), \bar{v}_{n}(0)\right)+C \mathbb{E}\left[\int_{0}^{t \wedge \bar{\tau}_{n}}\left(\int_{\mathcal{O}}\left|\log \left(\bar{u}_{n}(s, x)\right)+1\right|\left|\bar{u}_{n}(s, x)\right| d x\right)^{2} d s\right]^{\frac{1}{2}} .\right.
\end{aligned}
$$

In particular, there exists a constant $C>0$ such that we have for all $m \in \mathbb{N}$

$$
\mathbb{E} W\left(\bar{u}_{n}(t), \bar{v}_{n}(t)\right)=C\left(t+\mathbb{E} W\left(\bar{u}_{n}(0), \bar{v}_{n}(0)\right)\right) .
$$

Proof of Claim 3.5. Fix $n \in \mathbb{N}$. We will show the claim by applying the Itô formula to $W\left(\bar{u}_{n}, \bar{v}_{n}\right)(t)$. To be precise, in equation (3.13) one should replace before the Laplace operator $A$ by its Yosida approximation $A_{\varepsilon}=\frac{1}{\varepsilon}\left((i d-\varepsilon A)^{-1}-i d\right)$ and the divergence operator by intertwining a mollifier $\phi_{\varepsilon}$. One can then apply the Itô formula to $W\left(\bar{u}_{n}^{\varepsilon}, \bar{v}_{n}^{\varepsilon}\right)$, where $\left(\bar{u}_{n}^{\varepsilon}, \bar{v}_{n}^{\varepsilon}\right)$ are the solutions to system (3.13) in which the Laplace operator is replaced by its Yosida approximation and then take the limit $\varepsilon \rightarrow 0$ (see e.g. proof of Lemma 3.2 in [1]). Here, for any $\nu>0$ and $\delta \in \mathbb{R}$ we know that $\left|A-A_{\varepsilon}\right|_{L\left(H^{\delta+\nu}, H^{\delta}\right)} \rightarrow 0$ as $\varepsilon \rightarrow 0$. We now approximate the divergence operator. Let $\varphi \in C_{b}^{(2)}(\mathbb{R})$ be a function which is compactly supported, $\int \varphi(x) \mathrm{d} x=1$, and $\lim _{\epsilon \rightarrow 0} \varphi_{\epsilon}(x)=\lim _{\epsilon \rightarrow 0} \epsilon^{-1} \varphi(x / \epsilon)=\delta(x)$. Let $\operatorname{div}_{\varepsilon}(w):=\operatorname{div}\left(\varphi_{\varepsilon} \star w\right)$ where $\star$ denotes the convolution. Let

$$
\left\{\begin{aligned}
& d \bar{u}_{n}^{\varepsilon}(t)-\left[r_{u} A_{\varepsilon} \bar{u}_{n}^{\varepsilon}(t)-\chi \psi_{n}\left(h^{1}\left(\bar{u}_{n}^{\varepsilon}, t\right)\right) \psi_{n}\left(h^{2}\left(\bar{v}_{n}^{\varepsilon}, t\right)\right) \psi_{n}\left(h^{3}\left(\bar{v}_{n}^{\varepsilon}, t\right)\right)\right. \\
&\left.\times \operatorname{div}_{\varepsilon}\left(\bar{u}_{n}^{\varepsilon}(t) \nabla \bar{v}_{n}^{\varepsilon}(t)\right)+\gamma \bar{u}_{n}^{\varepsilon}(t)\right] d t=\bar{u}_{n}^{\varepsilon}(t) d \mathcal{W}_{1}(t) \\
& d \bar{v}_{n}^{\varepsilon}(t)-\left[r_{v} A_{\varepsilon} \bar{v}_{n}^{\varepsilon}(t)-\alpha \bar{v}_{n}^{\varepsilon}(t)\right] d t=\beta \bar{u}_{n}^{\varepsilon}(t) d t+\bar{v}_{n}^{\varepsilon}(t) d \mathcal{W}_{2}(t), \\
&\left(\bar{u}_{n}^{\varepsilon}(0), \bar{v}_{n}^{\varepsilon}(0)\right)=\left(u_{0}, v_{0}\right) .
\end{aligned}\right.
$$

Before applying the Itô-formula we have to reassure that the integrands belong to the appropriate space (see [6, Theorem 2.4] or [11, Chapter 4.4]). We note that

$$
W: L^{2}(\mathcal{O}) \times H^{1}(\mathcal{O}) \rightarrow \mathbb{R}
$$

is well-defined. Due to Lemma 3.1 for all $n \in \mathbb{N}$, there exists a constant $C(n)>0$ such that

$$
\begin{array}{ll}
\mathbb{E}\left[\sup _{0 \leq s \leq T}\left|\bar{u}_{n}^{\varepsilon}(s)\right|_{L^{2}}^{2}\right] \leq C(n), \quad \mathbb{E}\left[\sup _{0 \leq s \leq T}\left|\bar{u}_{n}(s)\right|_{L^{2}}^{2}\right] \leq C(n), \\
\mathbb{E}\left[\sup _{0 \leq s \leq T}\left|\nabla \bar{v}_{n}^{\varepsilon}(s)\right|_{L^{2}}^{2}\right] \leq C(n) \quad \mathbb{E}\left[\sup _{0 \leq s \leq T}\left|\bar{v}_{n}(s)\right|_{L^{2}}^{2}\right] \leq C(n) .
\end{array}
$$


As the integrands satisfy the conditions of Theorem 2.4 in [6], we can apply the Itô formula to the process $W\left(\bar{u}_{n}^{\varepsilon}(s), \bar{v}_{n}^{\varepsilon}(s)\right)$. One can show $W\left(\bar{u}_{n}^{\varepsilon}, \bar{v}_{n}^{\varepsilon}\right)(t) \rightarrow W\left(\bar{u}_{n}, \bar{v}_{n}\right)(t)$ as $\varepsilon \rightarrow 0$ for all $t \in[0, T]$. Indeed, by Claim 3.1, we know that there exists some $\alpha>0$ and a constant $C>0$ independent of $n \in \mathbb{N}$ such that

$$
\mathbb{E}\left[\sup _{0 \leq s \leq T}\left|\bar{u}_{n}^{\varepsilon}(s)\right|_{H^{\alpha}}^{2}\right] \leq C, \quad \mathbb{E}\left[\sup _{0 \leq s \leq T}\left|\bar{u}_{n}(s)\right|_{H^{\alpha}}^{2}\right] \leq C .
$$

We only deal with the following terms in the expression of $W\left(\bar{u}_{n}^{\varepsilon}, \bar{v}_{n}^{\varepsilon}\right)-W\left(\bar{u}_{n}, \bar{v}_{n}\right)$ namely

$$
\begin{aligned}
& \int_{\mathcal{O}}\left(\log \bar{u}_{n}(x)+1\right)\left(r_{u}\left(A_{\varepsilon}-A\right) \bar{u}_{n}(x)\right) d x \\
& \leq\left(\int_{\mathcal{O}}\left(\log \bar{u}_{n}(x)+1\right)^{2} d x\right)^{\frac{1}{2}}\left(\int_{\mathcal{O}}\left(r_{u}\left(A_{\varepsilon}-A\right) \bar{u}_{n}(x)\right)^{2} d x\right)^{\frac{1}{2}} \\
& \quad \leq\left(\int_{\mathcal{O}}\left(\log \bar{u}_{n}(x)+1\right)^{2} d x\right)^{\frac{1}{2}} r_{u}\left|A_{\varepsilon}-A\right|_{L\left(H^{\alpha}, L^{2}\right)}\left|\bar{u}_{n}\right|_{H^{\alpha}} \rightarrow 0
\end{aligned}
$$

and

$$
\left.\int_{\mathcal{O}}\left(\log \bar{u}_{n}(x)+1\right)\left(\operatorname{div}\left(\bar{u}_{n}(x) \nabla \bar{v}_{n}(x)\right)-\operatorname{div}\left(\left(\varphi_{\varepsilon} \star\left(\bar{u}_{n} \nabla \bar{v}_{n}\right)\right)(x)\right)\right)\right) d x \rightarrow 0
$$

as $\varepsilon \rightarrow 0$. Hence, $W\left(\bar{u}_{n}^{\varepsilon}, \bar{v}_{n}^{\varepsilon}\right)(t) \rightarrow W\left(\bar{u}_{n}, \bar{v}_{n}\right)(t)$ as $\varepsilon \rightarrow 0$ for all $t \in[0, T]$. Other terms in the expression $W\left(\bar{u}_{n}^{\varepsilon}, \bar{v}_{n}^{\varepsilon}\right)-W\left(\bar{u}_{n}, \bar{v}_{n}\right)$ can be handled in a similar way. However, for our convenience, we omit the technical details and apply the Itô formula directly to $W\left(\bar{u}_{n}, \bar{v}_{n}\right)(t)$. Also, we will use the representation of the Wiener process given by

$$
\mathcal{W}_{j}(t, x)=\sum_{k \in \mathbb{Z}} \psi_{k}^{\left(\delta_{j}\right)}(x) \beta_{k}^{(j)}(t), \quad j=1,2
$$

where $\psi_{k}^{\left(\delta_{j}\right)} ; j=1,2$ is defined in Remark 2.4 and $\left\{\beta_{k}^{j}: k \in \mathbb{Z}\right\} ; j=1,2$ are two families of mutually independent Brownian motions. We now start with the following calculations

$$
\begin{aligned}
& W\left(\bar{u}_{n}, \bar{v}_{n}\right)\left(t \wedge \bar{\tau}_{n}\right)-W\left(\bar{u}_{n}, \bar{v}_{n}\right)(0) \\
& =\underbrace{\int_{0}^{t \wedge \bar{\tau}_{n}}\left\langle\left(\log \bar{u}_{n}(s)+1\right), d \bar{u}_{n}(s)\right\rangle}_{I_{1}:=} \underbrace{-\rho \int_{0}^{t \wedge \bar{\tau}_{n}}\left\langle\bar{u}_{n}(s), d \bar{v}_{n}(s)\right\rangle+\left\langle\bar{v}_{n}(s), d \bar{u}_{n}(s)\right\rangle}_{I_{2}:=} \\
& \quad+\underbrace{\int_{0}^{t \wedge \bar{\tau}_{n}}\left\langle\nabla \bar{v}_{n}(s), \nabla d \bar{v}_{n}(s)\right\rangle}_{I_{3}:=}+\underbrace{\int_{0}^{t \wedge \bar{\tau}_{n}}\left\langle\bar{v}_{n}(s), d \bar{v}_{n}(s)\right\rangle} \\
& \quad+\frac{1}{2} \sum_{k \in \mathbb{Z}} \int_{0}^{t \wedge \bar{\tau}_{n}} \int_{\mathcal{O}} \bar{u}_{n}(s, x)\left(\psi_{k}^{\left(\delta_{1}\right)}(x)\right)^{2} d x d s \\
& \quad+\frac{C_{1}}{2} \sum_{k \in \mathbb{Z}} \int_{0}^{t \wedge \bar{\tau}_{n}}\left|\nabla\left(\bar{v}_{n}(s) \psi_{k}^{\left(\delta_{2}\right)}\right)\right|_{L^{2}}^{2} d s+\frac{C_{2}}{2} \sum_{k \in \mathbb{Z}} \int_{0}^{t \wedge \bar{\tau}_{n}}\left|\bar{v}_{n}(s) \psi_{k}^{\left(\delta_{2}\right)}\right|_{L^{2}}^{2} d s .
\end{aligned}
$$


Let us consider the first term in the right hand side of (3.58). Here, we obtain

$$
\begin{aligned}
I_{1}:=\int_{0}^{t \wedge \bar{\tau}_{n}} & \left\langle\left(\log \bar{u}_{n}(s)+1\right), d \bar{u}_{n}(s)\right\rangle \\
= & \int_{0}^{t \wedge \bar{\tau}_{n}} \int_{\mathcal{O}}\left(\log \bar{u}_{n}(s, x)+1\right)\left(r_{u} A \bar{u}_{n}(s, x)-\chi \operatorname{div}\left(\bar{u}_{n}(s, x) \nabla \bar{v}_{n}(s, x)\right)+\gamma \bar{u}_{n}(s, x)\right) d x d s \\
& +\sum_{k \in \mathbb{Z}} \int_{0}^{t \wedge \bar{\tau}_{n}} \int_{\mathcal{O}}\left(\log \bar{u}_{n}(s, x)+1\right) \bar{u}_{n}(s, x) \psi_{k}^{\left(\delta_{1}\right)}(x) d x d \beta_{k}^{(1)}(s) .
\end{aligned}
$$

Applying integration by parts one gets for the first two terms in (3.59)

$$
\begin{aligned}
\int_{0}^{t \wedge \bar{\tau}_{n}} & \int_{\mathcal{O}}\left(\log \bar{u}_{n}(s, x)+1\right)\left(r_{u} A \bar{u}_{n}(s, x)-\chi \operatorname{div}\left(\bar{u}_{n}(s, x) \nabla \bar{v}_{n}(s, x)\right)\right) d x d s \\
= & -\int_{0}^{t \wedge \bar{\tau}_{n}} \int_{\mathcal{O}} r_{u} \bar{u}_{n}^{-1}(s, x) \nabla \bar{u}_{n}(s, x)\left(\nabla \bar{u}_{n}(s, x)-\chi \bar{u}_{n}(s, x) \nabla \bar{v}_{n}(s, x)\right) d x d s \\
= & -\int_{0}^{t \wedge \bar{\tau}_{n}} 4 r_{u}\left|\nabla \sqrt{\bar{u}_{n}(s)}\right|_{L^{2}}^{2} d s-\chi \int_{0}^{t \wedge \bar{\tau}_{n}} \int_{\mathcal{O}} \bar{u}_{n}(s, x) A \bar{v}_{n}(s, x) d x d s .
\end{aligned}
$$

Applying the Burkholder-Davis-Gundy inequality (see (3.7)) and then the Hölder inequality give

$$
\begin{aligned}
& \mathbb{E}\left[\sup _{0 \leq s \leq t \wedge \bar{\tau}_{n}}\left|\sum_{k \in \mathbb{Z}} \int_{0}^{t \wedge \bar{\tau}_{n}} \int\left\langle\log \bar{u}_{n}(s)+1, \bar{u}_{n}(s) \psi_{k}^{\left(\delta_{1}\right)}\right\rangle d \beta_{k}^{(1)}(s)\right|\right] \\
& \leq 4 \mathbb{E}\left[\left(\sum_{k \in \mathbb{Z}} \int_{0}^{t \wedge \bar{\tau}_{n}}\left\langle\log \bar{u}_{n}(s)+1, \bar{u}_{n}(s) \psi_{k}^{\left(\delta_{1}\right)}\right\rangle^{2} d s\right)^{\frac{1}{2}}\right] \\
& \leq C \mathbb{E}\left[\int_{0}^{t \wedge \bar{\tau}_{n}}\left(\int_{\mathcal{O}}\left|\log \left(\bar{u}_{n}(s, x)\right)+1\right|\left|\bar{u}_{n}(s, x)\right| d x\right)^{2} d s\right]^{\frac{1}{2}} .
\end{aligned}
$$

Here, we used the fact that for $\delta_{1}>1$ there exists a $\sigma>\frac{1}{2}$ such that $H^{\delta_{1}}(\mathcal{O}) \hookrightarrow H^{\sigma}(\mathcal{O})$ is Hilbert-Schmidt and the embedding $H^{\sigma}(\mathcal{O}) \hookrightarrow L^{\infty}(\mathcal{O})$ is continuous.

We now estimate the next term $I_{2}$, that is, we deal with $\int_{\mathcal{O}} \bar{u}_{n}\left(t \wedge \bar{\tau}_{n}, x\right) \bar{v}_{n}\left(t \wedge \bar{\tau}_{n}, x\right) d x$. Applying again the Itô formula we obtain

$$
\begin{aligned}
I_{2}= & \int_{\mathcal{O}} \bar{u}_{n}\left(t \wedge \bar{\tau}_{n}, x\right) \bar{v}_{n}\left(t \wedge \bar{\tau}_{n}, x\right) d x-\int_{\mathcal{O}} u_{0}(x) v_{0}(x) d x \\
= & \int_{0}^{t \wedge \bar{\tau}_{n}} \int_{\mathcal{O}}\left(\bar{v}_{n}(s, x)\left(r_{u} A \bar{u}_{n}(s, x)-\chi \operatorname{div}\left(\bar{u}_{n}(s, x) \nabla \bar{v}_{n}(s, x)\right)-\gamma \bar{u}_{n}(s, x)\right) d x d s\right. \\
& +\int_{0}^{t \wedge \bar{\tau}_{n}} \int_{\mathcal{O}} \bar{u}_{n}(s, x)\left(r_{v} A \bar{v}_{n}(s, x)-\alpha \bar{v}_{n}(s, x)+\beta \bar{u}_{n}(s, x)\right) d x d s \\
& +\sum_{k \in \mathbb{Z}} \int_{0}^{t \wedge \bar{\tau}_{n}}\left\langle\bar{v}_{n}(s), \bar{u}_{n}(s) \psi_{k}^{\left(\delta_{1}\right)}\right\rangle d \beta_{k}^{(1)}(s)+\sum_{k \in \mathbb{Z}} \int_{0}^{t \wedge \bar{\tau}_{n}}\left\langle\bar{u}_{n}(s), \bar{v}_{n}(s) \psi_{k}^{\left(\delta_{2}\right)}\right\rangle d \beta_{k}^{(2)}(s) .
\end{aligned}
$$


Using integration by parts and the Neumann boundary conditions, we obtain

$$
\begin{gathered}
r_{u} \int_{\mathcal{O}} \bar{v}_{n}(s, x) A \bar{u}_{n}(s, x) d x=-r_{u} \int_{\mathcal{O}} \nabla \bar{v}_{n}(s, x) \nabla \bar{u}_{n}(s, x) d x=r_{u} \int_{\mathcal{O}} A \bar{v}_{n}(s, x) \bar{u}_{n}(s, x), \\
\chi \int_{\mathcal{O}} \bar{v}_{n}(s, x) \operatorname{div}\left(\bar{u}_{n}(s, x) \nabla \bar{v}_{n}(s, x)\right) d x=-\chi \int_{\mathcal{O}} \bar{u}_{n}(s, x)\left(\nabla \bar{v}_{n}(s, x)\right)^{2} d x .
\end{gathered}
$$

Proceeding in similar lines we obtain

$$
\begin{aligned}
& \int_{\mathcal{O}} \bar{v}_{n}\left(-\gamma \bar{u}_{n}\right)+\bar{u}_{n}(s, x)\left(-\alpha \bar{v}_{n}(s, x)+\beta \bar{u}_{n}(s, x)\right) d x \\
& =-(\alpha+\gamma) \int_{\mathcal{O}} \bar{u}_{n}(s, x) \bar{v}_{n}(s, x) d x+\beta \int_{\mathcal{O}} \bar{u}_{n}^{2}(s, x) d x .
\end{aligned}
$$

As before we know that for $\delta_{1}>1$ there exists a $\sigma>\frac{1}{2}$ such that $H^{\delta_{1}}(\mathcal{O}) \hookrightarrow H^{\sigma}(\mathcal{O})$ is HilbertSchmidt and the embedding $H^{\sigma}(\mathcal{O}) \hookrightarrow L^{\infty}(\mathcal{O})$ is continuous. This gives

$$
\begin{aligned}
& \mathbb{E}\left[\sup _{0 \leq s \leq t \wedge \bar{\tau}_{n}}\left|\sum_{k \in \mathbb{Z}} \int_{0}^{s}\left\langle\bar{v}_{n}(r), \bar{u}_{n}(r) \psi_{k}^{\left(\delta_{1}\right)}\right\rangle d \beta_{k}^{(1)}(r)\right|\right] \\
& \leq \mathbb{E}\left[\left(\sum_{k \in \mathbb{Z}} \int_{0}^{t \wedge \bar{\tau}_{n}}\left\langle\bar{v}_{n}(s), \bar{u}_{n}(s) \psi_{k}^{\left(\delta_{1}\right)}\right\rangle^{2} d s\right)^{\frac{1}{2}}\right] \leq C\left(\sum_{k \in \mathbb{Z}}\left|\psi_{k}^{\left(\delta_{1}\right)}\right|_{L^{\infty}}^{2} \mathbb{E}\left[\int_{0}^{t \wedge \bar{\tau}_{n}}\left\langle\bar{v}_{n}(s), \bar{u}_{n}(s)\right\rangle^{2} d s\right]\right)^{\frac{1}{2}} .
\end{aligned}
$$

Arguing in similar lines, we treat the following term. This gives

$$
\begin{aligned}
& \mathbb{E}\left[\sup _{0 \leq s \leq t \wedge \bar{\tau}_{n}}\left|\sum_{k \in \mathbb{Z}} \int_{0}^{s}\left\langle\bar{u}_{n}(r), \bar{v}_{n}(r) \psi_{k}^{\left(\delta_{2}\right)}\right\rangle d \beta_{k}^{(2)}(r)\right|\right] \\
& \leq \mathbb{E}\left[\left(\sum_{k \in \mathbb{Z}} \int_{0}^{t \wedge \bar{\tau}_{n}}\left\langle\bar{u}_{n}(s), \bar{v}_{n}(s) \psi_{k}^{\left(\delta_{2}\right)}\right\rangle^{2} d s\right)^{\frac{1}{2}}\right] \leq C\left(\sum_{k \in \mathbb{Z}}\left|\psi_{k}^{\left(\delta_{2}\right)}\right|_{L^{\infty}}^{2} \mathbb{E}\left[\int_{0}^{t \wedge \bar{\tau}_{n}}\left\langle\bar{u}_{n}(s), \bar{v}_{n}(s)\right\rangle^{2} d s\right]\right)^{\frac{1}{2}} .
\end{aligned}
$$

Tackling the terms $I_{3}$ and $I_{4}$ in (3.58) we obtain

$$
\begin{aligned}
& \frac{1}{2}\left|\nabla \bar{v}_{n}\left(t \wedge \bar{\tau}_{n}\right)\right|_{L^{2}}^{2}-\frac{1}{2}\left|\nabla v_{0}\right|_{L^{2}}^{2}+r_{v} \int_{0}^{t \wedge \bar{\tau}_{n}}\left|A \bar{v}_{n}(s)\right|_{L^{2}}^{2} d s \\
& \quad+\alpha \int_{0}^{t \wedge \bar{\tau}_{n}}\left|\nabla \bar{v}_{n}(s)\right|_{L^{2}}^{2} d s=-\beta \int_{0}^{t \wedge \bar{\tau}_{n}} \int_{\mathcal{O}} A \bar{v}_{n}(s, x) \bar{u}_{n}(s, x) d x d s \\
& \quad+\sum_{k \in \mathbb{Z}} \int_{0}^{t \wedge \bar{\tau}_{n}}\left\langle\nabla \bar{v}_{n}(s), \nabla\left(\bar{v}_{n}(s) \psi_{k}^{\left(\delta_{2}\right)}\right)\right\rangle d \beta_{k}^{(2)}(s)+\frac{1}{2} \sum_{k=1}^{\infty} \int_{0}^{t \wedge \bar{\tau}_{n}}\left|\nabla \bar{v}_{n}(s) \psi_{k}^{\left(\delta_{2}\right)}\right|_{L^{2}}^{2} d s
\end{aligned}
$$

and

$$
\begin{aligned}
& \frac{1}{2}\left|\bar{v}_{n}(t)\right|_{L^{2}}^{2}-\frac{1}{2}\left|v_{0}\right|_{L^{2}}^{2}+r_{v} \int_{0}^{t \wedge \bar{\tau}_{n}}\left|\nabla \bar{v}_{n}(s)\right|_{L^{2}}^{2} d s+\alpha \int_{0}^{t \wedge \bar{\tau}_{n}}\left|\bar{v}_{n}(s)\right|_{L^{2}}^{2} d s \\
& \quad=\beta \int_{0}^{t \wedge \bar{\tau}_{n}}\left\langle\bar{v}_{n}(s), \bar{u}_{n}(s)\right\rangle d s+\sum_{k=1}^{\infty} \int_{0}^{t \wedge \bar{\tau}_{n}}\left\langle\bar{v}_{n}(s),\left(\bar{v}_{n}(s) \psi_{k}^{\left(\delta_{2}\right)}\right)\right\rangle d \beta_{k}^{(2)}(s)
\end{aligned}
$$




$$
+\frac{1}{2} \sum_{k=1}^{\infty} \int_{0}^{t \wedge \bar{\tau}_{n}}\left|\bar{v}_{n}(s) \psi_{k}^{\left(\delta_{2}\right)}\right|_{L^{2}}^{2} d s .
$$

Again, by similarly consideration as above and the fact that $\delta_{2}>\frac{3}{2}$, we know

$$
\begin{aligned}
& \mathbb{E}\left[\sup _{0 \leq s \leq t \wedge \bar{\tau}_{n}}\left|\int_{0}^{s}\left\langle\nabla \bar{v}_{n}(s), \nabla\left(\bar{v}_{n}(s) \psi_{k}^{\left(\delta_{2}\right)}\right)\right\rangle d \beta_{k}^{(2)}(s)\right|\right] \\
& \leq C \mathbb{E}\left(\sum_{k=1}^{\infty}\left|\psi_{k}^{\left(\delta_{2}\right)}\right|_{L^{\infty}}^{2} \int_{0}^{t \wedge \bar{\tau}_{n}}\left|\nabla \bar{v}_{n}(s)\right|_{L^{2}}^{4} d s\right)^{\frac{1}{2}} \\
& \leq \frac{C_{1}}{2} \mathbb{E}\left[\sup _{0 \leq s \leq t \wedge \bar{\tau}_{n}}\left|\nabla \bar{v}_{n}(s)\right|_{L^{2}}^{2}\right]+C \mathbb{E}\left[\int_{0}^{t \wedge \bar{\tau}_{n}}\left|\nabla \bar{v}_{n}(s)\right|_{L^{2}}^{2} d s\right]
\end{aligned}
$$

and

$$
\begin{aligned}
\mathbb{E} & {\left[\sup _{0 \leq s \leq t \wedge \bar{\tau}_{n}}\left|\int_{0}^{s}\left\langle\bar{v}_{n}(s),\left(\bar{v}_{n}(s) \psi_{k}^{\left(\delta_{2}\right)}\right)\right\rangle d \beta_{k}^{(2)}(s)\right|\right] } \\
& \leq \mathbb{E}\left(\sum_{k=1}^{\infty} \int_{0}^{t \wedge \bar{\tau}_{n}}\left\langle\bar{v}_{n}(s), \bar{v}_{n}(s) \psi_{k}^{\left(\delta_{2}\right)}\right\rangle^{2} d s\right)^{\frac{1}{2}} \\
& \leq \frac{C_{2}}{2} \mathbb{E}\left[\sup _{0 \leq s \leq t \wedge \bar{\tau}_{n}}\left|\bar{v}_{n}(s)\right|_{L^{2}}^{2}\right]+C \mathbb{E}\left[\int_{0}^{t \wedge \bar{\tau}_{n}}\left|\bar{v}_{n}(s)\right|_{L^{2}}^{2} d s\right] .
\end{aligned}
$$

Recollecting altogether, we obtain at the end

$$
\begin{aligned}
\mathbb{E}\left[\sup _{0 \leq s \leq t \wedge \bar{\tau}_{n}}\left[W\left(\bar{u}_{n}(s), \bar{v}_{n}(s)\right)-W\left(u_{0}, v_{0}\right)\right]\right]+\mathbb{E} \int_{0}^{t \wedge \bar{\tau}_{n}}\left(4 r_{u}\left|\nabla \sqrt{\bar{u}_{n}(s)}\right|_{L^{2}}^{2}+C_{1} r_{v}\left|A \bar{v}_{n}(s)\right|_{L^{2}}^{2}\right. \\
\left.\quad+\left(C_{2} r_{v}+C_{1} \alpha\right)\left|\nabla \bar{v}_{n}(s)\right|_{L^{2}}^{2}+C_{2} \alpha\left|\bar{v}_{n}(s)\right|_{L^{2}}^{2}+\rho \beta\left|\bar{u}_{n}(s)\right|_{L^{2}}^{2}+\chi \rho\left\langle\bar{u}_{n}(s),\left(\nabla \bar{v}_{n}(s)\right)^{2}\right\rangle\right) d s \\
\leq \quad \mathbb{E} \int_{0}^{t \wedge \bar{\tau}_{n}}\left(\left(\rho \alpha+\rho \gamma+C_{2} \beta\right)\left\langle\bar{u}_{n}(s), \bar{v}_{n}(s)\right\rangle+\left(\chi+\rho r_{u}+\rho r_{v}+C_{1} \beta\right)\left|\left\langle u_{n}(s), A \bar{v}_{n}(s)\right\rangle\right|\right) d s \\
\quad+C \mathbb{E}\left[\int_{0}^{t \wedge \bar{\tau}_{n}}\left(\int_{\mathcal{O}}\left|\log \left(\bar{u}_{n}(s, x)\right)+1\right|\left|\bar{u}_{n}(s, x)\right| d x\right)^{2} d s\right]^{\frac{1}{2}} \\
\quad+\frac{C_{1}}{2} \mathbb{E}\left[\sup _{0 \leq s \leq t \wedge \bar{\tau}_{n}}\left|\nabla \bar{v}_{n}(s)\right|_{L^{2}}^{2}\right]+C \mathbb{E}\left[\int_{0}^{t \wedge \bar{\tau}_{n}}\left|\nabla \bar{v}_{n}(s)\right|_{L^{2}}^{2} d s\right]+C \mathbb{E}\left[\int_{0}^{t \wedge \bar{\tau}_{n}}\left\langle\bar{v}_{n}(s), \bar{u}_{n}(s)\right\rangle^{2} d s\right]^{\frac{1}{2}} \\
\left(3.60+\frac{C_{2}}{2} \mathbb{E}\left[\sup _{0 \leq s \leq t \wedge \bar{\tau}_{n}}\left|\bar{v}_{n}(s)\right|_{L^{2}}^{2}\right]+C \mathbb{E}\left[\int_{0}^{t \wedge \bar{\tau}_{n}}\left|\bar{v}_{n}(s)\right|_{L^{2}}^{2} d s\right] .\right.
\end{aligned}
$$

However, by the Young inequality we have for $\varepsilon_{0}=\frac{1}{4} \rho \beta /\left(\rho \alpha+\rho \gamma+C_{2} \beta\right)$

$$
\left\langle\bar{u}_{n}(s), \bar{v}_{n}(s)\right\rangle \leq C\left(\varepsilon_{1}\right)\left|\bar{v}_{n}(s)\right|_{L^{2}}^{2}+\varepsilon_{0}\left|\bar{u}_{n}(s)\right|_{L^{2}}^{2}
$$

and for $\varepsilon_{1}=\frac{1}{4} \rho \beta /\left(\chi+\rho r_{u}+\rho r_{v}+C_{1} \beta\right)$

$$
\left|\left\langle\bar{u}_{n}(s), A \bar{v}_{n}(s)\right\rangle\right| \leq C\left(\varepsilon_{1}\right)\left|A \bar{v}_{n}(s)\right|_{L^{2}}^{2}+\varepsilon_{1}\left|\bar{u}_{n}(s)\right|_{L^{2}}^{2},
$$


where $C\left(\varepsilon_{1}\right)=\left(\chi+\rho r_{u}+\rho r_{v}+C_{1} \beta\right) / \rho \beta$. In particular, the terms in the right hand side of (3.60) can be estimated in the following way

$$
\begin{aligned}
\mathbb{E}\left[\sup _{0 \leq s \leq t \wedge \bar{\tau}_{n}}\left[W\left(\bar{u}_{n}(s), \bar{v}_{n}(s)\right)-W\left(u_{0}, v_{0}\right)\right]\right] \\
\quad+\mathbb{E} \int_{0}^{t \wedge \bar{\tau}_{n}}\left(4 r_{u}\left|\nabla \sqrt{\bar{u}_{n}(s)}\right|_{L^{2}}^{2}+C_{1} r_{v}\left|A \bar{v}_{n}(s)\right|_{L^{2}}^{2}+\left(C_{2} r_{v}+C_{1} \alpha\right)\left|\nabla \bar{v}_{n}(s)\right|_{L^{2}}^{2}\right. \\
\left.\quad+C_{2} \alpha\left|\bar{v}_{n}(s)\right|_{L^{2}}^{2}+\frac{1}{4} \rho \beta\left|\bar{u}_{n}(s)\right|_{L^{2}}^{2}+\chi \rho\left\langle\bar{u}_{n}(s),\left(\nabla \bar{v}_{n}(s)\right)^{2}\right\rangle\right) d s \\
\leq \quad \mathbb{E} \int_{0}^{t \wedge \bar{\tau}_{n}}\left(C\left(\varepsilon_{0}\right)\left|\bar{v}_{n}(s)\right|_{L^{2}}^{2}+C\left(\varepsilon_{1}\right)\left|A \bar{v}_{n}(s)\right|_{L^{2}}^{2}\right) d s \\
\quad+C\left(t \wedge \bar{\tau}_{n}\right)^{\frac{1}{2}}\left(\mathbb{E}\left[\sup _{0 \leq s \leq t \wedge \bar{\tau}_{n}}\left(\int_{\mathcal{O}}\left|\log \left(\bar{u}_{n}(s, x)\right)+1\right|^{2}\left|\bar{u}_{n}(s, x)\right|^{2} d x\right)^{\frac{1}{2}}\right]\right) \\
\quad+\frac{C_{1}}{2} \mathbb{E}\left[\sup _{0 \leq s \leq t \wedge \bar{\tau}_{n}}\left|\nabla \bar{v}_{n}(s)\right|_{L^{2}}^{2}\right]+C \mathbb{E}\left[\int_{0}^{t \wedge \bar{\tau}_{n}}\left|\nabla \bar{v}_{n}(s)\right|_{L^{2}}^{2} d s\right] \\
\quad+\frac{C_{2}}{2} \mathbb{E}\left[\sup _{0 \leq s \leq t \wedge \bar{\tau}_{n}}\left|\bar{v}_{n}(s)\right|_{L^{2}}^{2}\right]+C \mathbb{E}\left[\int_{0}^{t \wedge \bar{\tau}_{n}}\left|\bar{v}_{n}(s)\right|_{L^{2}}^{2} d s\right] .
\end{aligned}
$$

Using Gronwall inequality we get the follwing

$$
\begin{aligned}
& \left.\mathbb{E} \sup _{0 \leq s \leq t \wedge \bar{\tau}_{n}}\left[W\left(\bar{u}_{n}(s), \bar{v}_{n}(s)\right)-W\left(u_{0}, v_{0}\right)\right]\right] \\
& \quad+\mathbb{E} \int_{0}^{t \wedge \bar{\tau}_{n}}\left(4 r_{u}\left|\nabla \sqrt{\bar{u}_{n}(s)}\right|_{L^{2}}^{2}+\left(C_{1} r_{v}-C\left(\varepsilon_{1}\right)\left|A \bar{v}_{n}(s)\right|_{L^{2}}^{2}+\left(C_{2} r_{v}+C_{1} \alpha\right)\left|\nabla \bar{v}_{n}(s)\right|_{L^{2}}^{2}\right.\right. \\
& \left.\quad+C_{2} \alpha\left|\bar{v}_{n}(s)\right|_{L^{2}}^{2}+\frac{1}{2} \rho \beta\left|\bar{u}_{n}(s)\right|_{L^{2}}^{2}+\chi \rho\left\langle\bar{u}_{n}(s),\left(\nabla \bar{v}_{n}(s)\right)^{2}\right\rangle\right) d s \\
& \leq \mathbb{E} \int_{0}^{t \wedge \bar{\tau}_{n}} C\left(\varepsilon_{0}\right)\left|\bar{v}_{n}(s)\right|_{L^{2}}^{2} d s+C \mathbb{E} \int_{0}^{t \wedge \bar{\tau}_{n}}\left[\left|\nabla \bar{v}_{n}(s)\right|_{L^{2}}^{2}\right] d s \\
& \quad+C \mathbb{E}\left[\int_{0}^{t \wedge \bar{\tau}_{n}}\left(\int_{\mathcal{O}}\left|\log \left(\bar{u}_{n}(s, x)\right)+1\right|\left|\bar{u}_{n}(s, x)\right| d x\right)^{2} d s\right]^{\frac{1}{2}} .
\end{aligned}
$$

In the above inequality we choose $C_{1}>\left(r_{u}+r_{v}\right) / \beta r_{v}$ and $\rho>\left(\chi+C_{1} \beta\right) /\left(C_{1} \beta r_{v}-r_{v}-r_{u}\right)$, then $C_{1} r_{v}-C\left(\varepsilon_{1}\right)>0$. This completes the proof of Claim 3.5 .

It remains to estimate the two entities $\left|\bar{u}_{n}(\cdot)\right|_{\text {LlogL }}$ and $\left|\nabla \bar{v}_{n}(\cdot)\right|_{L^{2}}$ in the time interval $\left[\bar{\tau}_{n}, T\right]$. This we can do using standard estimates for the heat equation with multiplicative Wiener noise. In particular, applying the Itô formula we obtain for $u \in \operatorname{Llog} L(\mathcal{O})$ and $v \in H^{1}(\mathcal{O})$

$$
\mathcal{E}(u, v):=\int_{\mathcal{O}} u(x) \log (u(x)) d x+C_{1}|\nabla v|_{L^{2}}^{2},
$$


and by elementary calculations we obtain

$$
\begin{aligned}
& \bar{u}_{n}(T) \log \left(\bar{u}_{n}(T)\right)=\bar{u}_{n}\left(\bar{\tau}_{n}\right) \log \left(\bar{u}_{n}\left(\bar{\tau}_{n}\right)\right)+\int_{\bar{\tau}_{n}}^{T} \int_{\mathcal{O}} A \bar{u}_{n}(t, x)\left(\log \left(\bar{u}_{n}(t, x)\right)+1\right) d x d t \\
& \quad+\int_{\bar{\tau}_{n}}^{t} \sum_{k \in \mathbb{Z}}\left\langle\log \left(1+\bar{u}_{n}(s)\right) \bar{u}_{n}(s) \psi_{k}^{\left(\delta_{1}\right)}\right\rangle d \beta_{k}^{(1)}(s)+\frac{1}{2} \sum_{k \in \mathbb{Z}} \int_{\bar{\tau}_{n}}^{t} \int_{\mathcal{O}} \bar{u}_{n}(s, x)\left(\psi_{k}^{\left(\delta_{1}\right)}(x)\right)^{2} d x d s .
\end{aligned}
$$

Similar computations works also for $\left|\nabla \bar{v}_{n}(\cdot)\right|_{L^{2}}^{2}$. In particular, we obtain

$$
\begin{aligned}
& \left|\nabla \bar{v}_{n}(T)\right|_{L^{2}}^{2}-\left|\nabla \bar{v}_{n}\left(\bar{\tau}_{n}\right)\right|_{L^{2}}^{2}-\int_{\bar{\tau}_{n}}^{T}\left\langle A \bar{v}_{n}(s), A \bar{v}_{n}(s)\right\rangle d s \\
& \quad=\int_{\bar{\tau}_{n}}^{T} \sum_{k \in \mathbb{Z}}\left\langle\nabla \bar{v}_{n}(s), \nabla \bar{v}_{n}(s) \psi_{k}^{\left(\delta_{2}\right)}\right\rangle d \beta_{k}^{(2)}(s)+\frac{1}{2} \sum_{k \in \mathbb{Z}} \int_{\bar{\tau}_{n}}^{T}\left|\nabla\left(\bar{v}_{n}(s) \psi_{k}^{\left(\delta_{2}\right)}\right)\right|_{L^{2}}^{2} d s .
\end{aligned}
$$

The stochastic integrals can be estimated again by (3.7) and (3.9).

Let us summarize. We have shown that there exists a constant $C>0$ such that for any $t \in[0, T]$ we have

$$
\mathbb{E}\left[\sup _{0 \leq s \leq t}\left[\mathcal{E}\left(\bar{u}_{n}(s), \bar{v}_{n}(s)\right)-\mathcal{E}\left(u(0), \bar{v}_{n}(0)\right]\right] \leq C \int_{0}^{t} \mathbb{E}\left[\sup _{0 \leq \sigma \leq s} \mathcal{E}\left(\bar{v}_{n}(\sigma), \bar{u}_{n}(\sigma)\right) d s\right] .\right.
$$

The Grownwal inequality gives

$$
\mathbb{E}\left[\sup _{0 \leq s \leq t}\left[\left|\nabla \bar{v}_{n}(s)\right|_{L^{2}}^{2}+\left|\bar{u}_{n}(s)\right|_{\operatorname{Llog} L}\right]\right] \leq C\left(\mathbb{E} \mathcal{E}\left(u_{0}, v_{0}\right)+1\right) .
$$

In addition, we know that $\mathbb{E} W\left(u_{0}, v_{0}\right) \leq \mathbb{E}\left[\left|\nabla v_{0}\right|_{L^{2}}^{2}+\left|u_{0}\right|_{\text {LlogL }}\right]$. This completes the proof of Claim 3.4.

Step V: In the final step, we will show that $\mathbb{P}$-a.s. a martingale solution to (3.53) exists.

Claim 3.6. There exists a measurable set $\tilde{\Omega} \subset \Omega$ with $\mathbb{P}(\tilde{\Omega})=1$ such that on $\tilde{\Omega}$ a martingale solution $(u, v)$ of (3.11) exists.

Proof. For any $m \in \mathbb{N}$ let us define the set

$$
A_{m}:=\left\{\omega \in \Omega: \tau_{m}^{*} \geq T\right\} .
$$

It can be clearly observed that there exists a progressively measurable process $(u, v)$ over $\mathfrak{A}$ such that $(u, v)$ solves $\mathbb{P}$-a.s. the integral equation given by (3.11) up to time $T$. In particular, we have for the conditioned probability

$$
\mathbb{P}\left(\{\text { there exists a solution }(u, v) \text { to }(3.11)\} \mid A_{m}\right)=1 \text {. }
$$

Then, it is elementary to verify, that

$\mathbb{P}(\{$ there exists a solution to $(\underline{3.11})\})=\lim _{m \rightarrow \infty} \mathbb{P}\left(\{\right.$ there exists a solution $(u, v)$ to (3.11) $\left.\} \mid A_{m}\right) \mathbb{P}\left(A_{m}\right)$.

Since $\mathbb{P}\left(\{\right.$ a solution $(u, v)$ to (3.11) exists $\left.\} \mid A_{m}\right)=1$, it remains to show that $\lim _{m \rightarrow \infty} \mathbb{P}\left(A_{m}\right)=$ 1. Then, as $A_{m} \supset A_{m+1}$, it follows that

$$
\mathbb{P}(\{\text { there exists solution to } 3.110)=1 \text {. }
$$


Since there exists a constant $C(T)>0$ such that

$$
\mathbb{E}\left[\sup _{0 \leq t \leq T}\left|\bar{u}_{n}(t)\right|_{L^{1}}\right]+\mathbb{E}\left[\sup _{0 \leq t \leq T}\left|\nabla \bar{v}_{n}(t)\right|_{L^{2}}^{2}\right]+\mathbb{E}\left[\int_{0}^{T}\left|\nabla \bar{v}_{n}(t)\right|_{H^{1}}^{2} d t\right] \leq C(T) .
$$

we deduce

$$
\mathbb{P}\left(\Omega \backslash A_{m}\right) \leq \frac{C(T)}{m} \rightarrow 0 .
$$

The solution process is well defined on $\tilde{\Omega}=\lim _{m \rightarrow \infty} \mathcal{A}_{m}$, where $\mathbb{P}(\tilde{\Omega})=1$.

This completes the proof of Theorem 2.6.

\section{Appendix A. Proof of Corollary 2.7}

As mentioned in the introduction, we move the proof of Corollary 2.7 to the appendix. For the convenience of the reader, we will shortly introduce the results of [7] and [8], respectively, which we use within the proof of the main result. For simplicity, we do not state the results in their most general forms.

First, let us state the setting within our framework.

Assumption A.1. Let $E$ be a Hilbert space and let $\mathcal{A}$ be a linear map on $E$ satisfying the following conditions.

a) $-\mathcal{A}$ is a positive operator on $E$ with compact resolvent. In particular, there exists $M>0$ such that

$$
\left\|(\mathcal{A}+\lambda)^{-1}\right\| \leq \frac{M}{1+\lambda}, \text { for any } \lambda \geq 0
$$

b) infinitesimal generator of an analytic semigroup of contraction type in $E$.

c) $\mathcal{A}$ has the BIP (bounded imaginary power) property, i.e. there exist some constants $K>0$ and $\lambda \in\left[0, \frac{\pi}{2}\right)$ such that

$$
\left\|\mathcal{A}^{i s}\right\| \leq K e^{\vartheta|s|}, s \in \mathbb{R} .
$$

Let us fix two real numbers $q \in(1, \infty)$ and $\mu \geq 0$. Similarly to Brzeźniak and Gạtarek [8] we define a linear operator $\mathbb{A}$ by the formula

$$
\begin{aligned}
D(\mathbb{A}) & :=\left\{u \in L^{q}(0, T ; E): \mathcal{A} u \in L^{q}(0, T ; E)\right\}, \\
\mathbb{A} u & :=\{(0, T) \ni t \mapsto \mathcal{A}(u(t)) \in E\}, \quad u \in D(\mathbb{A}) .
\end{aligned}
$$

It is known, see [12], that if $\mathcal{A}+\mu I$ satisfies Assumption A.1, then so does $\mathbb{A}+\mu I$. With $q$ and $\mathcal{B}$ as above we define two operators $\mathbb{B}$ and $\Lambda$, see [8], by

$$
\begin{aligned}
\mathbb{B} u & :=u^{\prime}, \quad u \in D(\mathbb{B}):=H_{0}^{1, q}(0, T ; E) . \\
\Lambda & :=\mathbb{B}+\mathbb{A}, \quad D(\Lambda):=D(\mathbb{B}) \cap D(\mathbb{A}) .
\end{aligned}
$$

Next, by [12] and [15], since $\Lambda=\mathbb{B}-\mu I+\mathbb{A}+\mu I$, we deduce that $\Lambda$ is a positive operator. In particular, $\Lambda$ has a bounded inverse. The domain $D(\Lambda)$ of $\Lambda$ endowed with the 'graph' norm

$$
\|u\|=\left\{\int_{0}^{T}\left|u^{\prime}(s)\right|^{q} d s+\int_{0}^{T}|\mathcal{A} u(s)|^{q} d s\right\}^{\frac{1}{q}}
$$

\footnotetext{
${ }^{2}$ See Section I.14.1 in Triebel's monograph 33 .
} 
is a Banach space. Let us present two results on the fractional powers of the operator $\Lambda$, see [7] for the proof.

Proposition A.2. Assume that Assumption A.1 is satisfied. Then, for any $\bar{\alpha} \in(0,1]$, the operator $\Lambda^{-\bar{\alpha}}$ is a bounded linear operator in $L^{q}(0, T ; E)$, and for $\eta \in L^{q}(0, T ; E)$,

$$
\left(\Lambda^{-\bar{\alpha}} \eta\right)(t)=\frac{1}{\Gamma(\bar{\alpha})} \int_{0}^{t}(t-s)^{\bar{\alpha}-1} e^{-(t-s) \mathcal{A}} \eta(s) d s, \quad t \in[0, T] .
$$

Lemma A.3. (Compare with Lemma 2.4 of [8]) Let Assumption A.1 be satisfied. Suppose that the positive numbers $\bar{\alpha}, \beta, \delta$ and $q>1$ satisfy

$$
\bar{\alpha}-\frac{1}{q}+\gamma>\beta+\delta
$$

If $T \in(0, \infty)$, then the operator

$$
\Lambda^{-\bar{\alpha}}: \mathbb{L}^{q}\left(0, T ; D\left(\mathcal{A}^{\gamma}\right)\right) \rightarrow C_{b}^{\beta}\left([0, T] ; D\left(\mathcal{A}^{\delta}\right)\right)
$$

is bounded.

Finally, we present slight modifications of Proposition 2.2 and Theorem 2.6 from [7]. Corollary A.6] is related to Lemma 3.3 of [34].

Theorem A.4. Let Assumption A.1 be satisfied. Assume that $\bar{\alpha} \in(0,1]$ and $\delta, \rho \geq 0$ are such that

$$
\bar{\alpha}-\frac{1}{q}+\rho-\delta>-\frac{1}{r}
$$

Then the operator

$$
\mathcal{A}^{\delta} \Lambda^{-\bar{\alpha}} \mathcal{A}^{-\rho}: L^{q}(0, T ; E) \rightarrow L^{r}(0, T ; E)
$$

is bounded. Moreover, if the operator $\mathcal{A}^{-1}: E \rightarrow E$ is compact, then the operator in (A.1) is compact.

Remark A.5. In view of Theorem A.4

$$
\Lambda^{-1}: L^{p}(0, T ; E) \rightarrow L^{p}(0, T ; E)
$$

is a well defined bounded linear operator for $p \in[1, \infty)$. Observe, that $\Lambda^{-1} \eta$ is the solution to the Cauchy problem

$$
\left\{\begin{aligned}
\dot{u}(t) & =\mathcal{A} u(t)+\eta(t) \\
u(0) & =0
\end{aligned}\right.
$$

Corollary A.6. Assume the first set of assumptions of Theorem A.4 are satisfied. Assume that three non-negative numbers $\bar{\alpha}, \beta, \delta$ satisfy the following condition

$$
\bar{\alpha}-\frac{1}{q}>\beta+\delta \text {. }
$$

Then the operator $\Lambda^{-\bar{\alpha}}: L^{q}(0, T ; E) \rightarrow C_{b}^{\beta}\left([0, T] ; D\left(\mathcal{A}^{\delta}\right)\right)$ is bounded. Moreover, if the operator $\mathcal{A}^{-1}: E \rightarrow E$ is compact, then the operator $\Lambda^{-\bar{\alpha}}: L^{q}(0, T ; E) \rightarrow C_{b}^{\beta}\left([0, T] ; D\left(\mathcal{A}^{\delta}\right)\right)$ is also compact.

In particular, if $\bar{\alpha}>\frac{1}{q}$ and the operator $\mathcal{A}^{-1}: E \rightarrow E$ is compact, the map $\Lambda^{-\bar{\alpha}}: L^{q}(0, T ; E) \rightarrow$ $C_{b}^{0}([0, T] ; E)$ is compact. 
Let $\mathfrak{A}=(\Omega, \mathcal{F}, \mathbb{F}, \mathbb{P})$ be a complete probability space and $\mathbb{F}=\left(\mathcal{F}_{t}\right)_{t \in[0, T]}$ be a filtration satisfying the usual conditions. Let $H$ and $H_{1}$ be two Hilbert spaces, $\mathcal{W}$ be an $H_{1}$-valued cylindrical Wiener process, $L_{H S}\left(H_{1}, H\right)$ be the space of linear operators belonging to $L\left(H_{1}, H\right)$ with finite Hilbert-Schmidt norm, and

$$
\begin{gathered}
\mathcal{N}_{\mathfrak{A}}^{p}(0, T ; H):=\{\xi:[0, T] \times \Omega \rightarrow H \mid \xi \text { is progressively measurable over } \mathfrak{A} \\
\text { and } \left.\mathbb{E} \int_{0}^{T}|\xi(s)|_{L_{H S}\left(H_{1}, H\right)} d s<\infty\right\} .
\end{gathered}
$$

To handle the stochastic convolution, let us define for a process $\xi \in \mathcal{N}_{\mathfrak{A}}^{2}(0, T ; H)$ the operator $\mathfrak{S}_{A}$ by

$$
\mathfrak{S}_{\mathcal{A}} \xi(t)=\int_{0}^{t} e^{-(t-s) \mathcal{A}} \xi(s) d \mathcal{W}(s)
$$

Corollary A.7. (Compare with Corollary 3.5 of [7, p. 266] and [34, Lemma 3.3]) If non-negative numbers $\beta, \delta$ and $\rho$ satisfy the following

$$
\beta+\delta+\frac{1}{p}<\frac{1}{2}-\rho
$$

then a process $x(t), t \in[0, T]$ belonging to $\mathcal{N}_{\mathfrak{A}}^{q}\left(0, T ; \mathcal{A}^{-\rho} H\right)$ possesses a modification $\tilde{x}(t), t \in$ $[0, T]$ such that

$$
\tilde{x} \in C_{b}^{\beta}\left([0, T] ; D\left(\mathcal{A}^{\delta}\right)\right), \quad \text { a.s. },
$$

and there exists some constant $C_{T}>0$ such that

$$
\mathbb{E}\left[\|\tilde{x}\|_{\mathcal{C}_{b}^{\beta}\left([0, T] ; D\left(\mathcal{A}^{\delta}\right)\right)}^{p}\right] \leq C_{T} \mathbb{E}\left[\int_{0}^{T}\left\|\mathcal{A}^{-\rho} x(s)\right\|_{L_{H S}\left(H_{1}, H\right)}^{p} d s\right] .
$$

Now we can start with the proof of Corollary 2.7

Proof of Corollary 2.7. Let us define the operator $\mathfrak{F}_{r_{u} A+\gamma I}: L^{q}\left(0, T ; L^{2}(\mathcal{O})\right) \rightarrow L^{q}\left(0, T ; L^{2}(\mathcal{O})\right)$ for $q>1$ by

$$
\left(\mathfrak{F}_{r_{u} A+\gamma I} \eta\right)(t)=\int_{0}^{t} e^{(t-s)\left(r_{u} A+\gamma I\right)} \eta(s) d s, \quad t \in[0, T], \eta \in L^{q}\left(0, T ; L^{2}(\mathcal{O})\right) .
$$

We observe that the operator $\mathfrak{F}_{r_{u} A+\gamma I}$ is identical to the operator $\Lambda^{-1}$ of Proposition A.2, We note that the process $u$ is given by for $t \in[0, T]$

$$
\begin{aligned}
u(t) & =e^{t\left(r_{u} A+\gamma I\right)} u_{0}+\int_{0}^{t} e^{(t-s)\left(r_{u} A+\gamma I\right)} \eta(s) d s+\int_{0}^{t} e^{(t-s)\left(r_{u} A+\gamma I\right)} u(s) d W_{1}(s) \\
& =e^{t\left(r_{u} A+\gamma I\right)} u_{0}+\left(\mathfrak{F}_{r_{u} A+\gamma I} \eta\right)(t)+\mathfrak{S}_{r_{u} A+\gamma I}(u)(t)
\end{aligned}
$$

with $\eta=\operatorname{div}(u(\cdot) \nabla v(\cdot))$ and

$$
\begin{aligned}
v(t) & =e^{t\left(r_{v} A-\alpha I\right)} v_{0}+\int_{0}^{t} e^{(t-s)\left(r_{u} A-\alpha I\right)} u(s) d s+\int_{0}^{t} e^{(t-s)\left(r_{v} A-\alpha I\right)} v(s) d W_{2}(s) \\
& =e^{t\left(r_{v} A-\alpha I\right)} v_{0}+\left(\mathfrak{F}_{r_{u} A+\gamma I} u\right)(t)+\left(\mathfrak{S}_{r_{u} A-\alpha I} v\right)(t) .
\end{aligned}
$$

First, we tackle $u$. We note that the operator $r_{u} A+\gamma I$ satisfies Assumption A.1. In Lemma A.3 it can be shown that the operator $\mathcal{A}$ satisfying the assumptions A.1. We choose $\bar{\alpha}, q, \rho, \beta, \delta$ 
appropriately in Lemma A.3 so that the results of Lemma A.3 hold for the operator $\mathcal{A}=r_{u} A+\gamma I$. Let us choose $\bar{\alpha}=1,1<q, \rho<-3 / 4$ and $\beta, \delta>0$ such that

$$
0<\beta+\delta<1+\rho-\frac{1}{q} .
$$

By Lemma A.3, we note that for $\eta \in L^{q}\left(0, T ; H^{2 \rho}\right), \mathfrak{F}_{\mathcal{A}} \eta \in C_{b}^{\beta}\left([0, T] ; H^{2 \delta}\right)$, i.e., in particular,

$$
\left\|\mathfrak{F}_{\mathcal{A}} \eta\right\|_{C_{b}^{\beta}\left([0, T] ; H^{2 \delta}\right)} \leq C\|\eta\|_{L^{q}\left(0, T ; H^{2 \rho}\right)} .
$$

As $\eta(\cdot)=\operatorname{div}(u(\cdot) \nabla v(\cdot))$, then, by the Sobolev embedding $L^{1}(\mathcal{O}) \hookrightarrow H^{2 \rho+1}(\mathcal{O})$, as $2 \rho+1<-\frac{1}{2}$. Therefore as $H^{\theta}(\mathcal{O}) \hookrightarrow L^{\infty}(\mathcal{O})$ for $\theta>1 / 2$, and using the interpolation inequality for $H^{\theta}(\mathcal{O})=$ $\left[H^{1}(\mathcal{O}), L^{2}(\mathcal{O})\right]_{\theta}$ we obtain

$$
\begin{aligned}
|\operatorname{div}(u(s) \nabla v(s))|_{H^{2 \rho}} & \leq|u(s) \nabla v(s)|_{H^{2 \rho+1}} \leq|u(s) \nabla v(s)|_{L^{1}} \leq|u(s)|_{L^{1}}|\nabla v(s)|_{L^{\infty}} \\
& \leq|u(s)|_{L^{1}}|\nabla v(s)|_{L^{2}}^{1-\theta}|\nabla v(s)|_{H^{1}}^{\theta} .
\end{aligned}
$$

For any $q \in(2,4)$ there exists a constant $C_{q}>0$ such that the $L^{q}\left(0, T ; H^{2 \rho}(\mathcal{O})\right)$-norm of the function $\eta$ can be estimated by

$$
\|\eta\|_{L^{q}\left(0, T ; H^{2 \rho}\right)} \leq C_{q} \sup _{0 \leq s \leq T}|u(s)|_{L^{1}} \sup _{0 \leq s \leq T}|\nabla v(s)|_{L^{2}}^{1-\theta}\left(\int_{0}^{T}|\nabla v(s)|_{H^{1}}^{2} d s\right)^{\frac{1}{q}},
$$

where $\theta=2 / q$. In particular, the Hölder inequality gives for $\varrho=2 / 3$

$$
\begin{aligned}
& \mathbb{E}\left[\|\eta\|_{L^{q}\left(0, T ; H^{2 \rho}\right)}^{p \varrho}\right] \\
& \leq\left(\mathbb{E}\left[\sup _{0 \leq s \leq T}|u(s)|_{L^{1}}^{p}\right]\right)^{\varrho}\left(\mathbb{E}\left[\sup _{0 \leq s \leq T}|\nabla v(s)|_{L^{2}}^{2 p}\right]\right)^{\frac{(1-\theta) \varrho}{2}}\left(\mathbb{E}\left[\int_{0}^{T}|\nabla v(s)|_{H^{1}}^{2} d s\right]^{p}\right)^{\frac{\varrho}{q}} .
\end{aligned}
$$

By estimate (2.4) we know that the right hand side of (A.3) is bounded for $p \geq 1$. Using (A.2) and (A.3) we obtain $\mathbb{E}\left\|\mathfrak{F}_{\mathcal{A}} \eta\right\|_{C_{b}^{\beta}\left([0, T] ; H^{2 \delta}\right)}^{p \varrho}$ is finite. It remains to analyse the stochastic term $\mathfrak{S}_{\mathcal{A}}(u)$. Here, we apply Corollary A.7 for $p>4, \beta, \delta>0 \varrho>0$ for $\beta+\delta+\frac{1}{p}<\frac{1}{2}-\rho, \rho>\frac{1}{4}$ and (3.7) to get

$$
\mathbb{E}\left[\left\|\mathfrak{S}_{\mathcal{A}} u\right\|_{C_{b}^{\beta}\left([0, T] ; H^{2 \delta}\right)}^{p \varrho}\right] \leq C\left(\mathbb{E}\left[\sup _{0 \leq s \leq T}|u(s)|_{H^{-2 \rho}}^{p}\right]\right)^{\varrho} \leq C\left(\mathbb{E}\left[\sup _{0 \leq s \leq T}|u(s)|_{L^{1}}^{p}\right]\right)^{\varrho} .
$$

By Theorem 2.6, estimate (2.5) we observe that the right hand side of (A.3) is finite. Hence we conclude that

$$
\mathbb{E}\left[\left\|u-e^{(\cdot)\left(r_{u} A+\gamma I\right)} u_{0}\right\|_{C_{b}^{\beta}\left([0, T] ; H^{2 \delta}\right)}^{\varrho p}\right]<\infty .
$$

This proves the aasertion for $u$.

It remains to verify the assertion for $v$. Let us choose $\bar{\alpha}=1,1<q, \rho<-3 / 4$ and $\beta, \delta>0$ in Lemmma A.3 such that

We now have the estimate

$$
0<\beta+\delta<1+\rho-\frac{1}{q}
$$

$$
\left\|\mathfrak{F}_{r_{v} A-\alpha I} \nabla u\right\|_{C_{b}^{\beta}\left([0, T] ; H^{2 \delta}\right)} \leq C\|\nabla u\|_{L^{q}\left(0, T ; H^{2 \rho}\right)} \leq C\|u\|_{L^{q}\left(0, T ; H^{2 \rho+1}\right)} \leq C\|u\|_{L^{q}\left(0, T ; L^{1}\right)}
$$


Hence,

$$
\mathbb{E}\left[\left\|\mathfrak{F}_{r_{v} A-\alpha I} \nabla u\right\|_{C_{b}^{\beta}\left([0, T] ; H^{2 \delta}\right)}^{p}\right] \leq C \mathbb{E}\left[\sup _{0 \leq s \leq T}|u(s)|_{L^{1}}^{p}\right] .
$$

To analyse the stochastic term, we apply Corollary A.7 for $p>4, \beta, \delta>0$ for $\beta+\delta+\frac{1}{p}<\frac{1}{2}-\rho$, $\rho>\frac{1}{4}$, and (3.7) to get

$$
\begin{aligned}
& \mathbb{E}\left[\left\|\mathfrak{S}_{r_{v} A-\alpha I} \nabla v\right\|_{C_{b}^{\beta}\left([0, T] ; H^{2 \delta}\right)}^{p}\right] \leq C \mathbb{E}\left[\sup _{0 \leq s \leq T}|\nabla v(s)|_{H^{-2 \rho}}^{p}\right] \\
& \leq C\left(\mathbb{E}\left[\sup _{0 \leq s \leq T}|\nabla v(s)|_{L^{2}}^{2 p}\right]\right)^{1 / 2}
\end{aligned}
$$

By Theorem 2.6, estimate (2.5) we observe that right hand side of (A.4) is finite. Hence we conclude that

$$
\mathbb{E}\left[\left\|\nabla v-e^{(\cdot)\left(r_{v} A-\alpha I\right)} \nabla v_{0}\right\|_{C_{b}^{\beta}\left([0, T] ; H^{2 \delta}\right)}^{p}\right]<\infty .
$$

This gives the assertion for $v$.

\section{REFERENCES}

[1] V. Barbu, C. Marinelli. Strong solutions for stochastic porous media equations with jumps. Infin. Dimens. Anal. Quantum Probab. Relat. Top. 12 (2009), no. 3, 413426.

[2] V. Barbu, G. Da Prato, and M. Röckner. Stochastic porous media equations., volume 2163. Cham: Springer, 2016.

[3] N. Bellomo, A. Bellouquid, Y. Tao, and M. Winkler. Toward a mathematical theory of Keller-Segel models of pattern formation in biological tissues. Math. Models Methods Appl. Sci., 25:1663-1763, 2015.

[4] C. Bennett and R. Sharpley. Interpolation of operators. Boston, MA etc.: Academic Press, Inc., 1988.

[5] P. Biler. Mathematical challenges in the theory of chemotaxis. Ann. Math. Sil., 32:43-63, 2018.

[6] Z. Brzeźniak,J. van Neerven, M. Veraar, and L. Weis. Itôs formula in UMD Banach spaces and regularity of solutions of the Zakai equation. J. Differ. Equations, 245:30-58, 2008.

[7] Z. Brzeźniak. On stochastic convolution in Banach spaces and applications. Stochastics Stochastics Rep., 61:245-295, 1997.

[8] Z. Brzeźniak and D. Gątarek. Martingale solutions and invariant measures for stochastic evolution equations in Banach spaces. Stochastic Process. Appl., 84:187-225, 1999.

[9] Z. Brzeźniak, E. Hausenblas, and P. Razafimandimby. Stochastic reaction-diffusion equations driven by jump processes. Potential Anal., 49:131-201, 2018.

[10] R. Dalang and L. Quer-Sardanyons. Stochastic integrals for spde's: a comparison. Expo. Math., 29:67-109, 2011.

[11] G. Da Prato and J. Zabczyk. Stochastic equations in infinite dimensions. 2nd ed. Cambridge: Cambridge University Press, 2nd ed. edition, 2014.

[12] G. Dore and A. Venni. On the closedness of the sum of two closed operators. Math. Z., 196:189-201, 1987.

[13] J. Duan and W. Wang. Effective dynamics of stochastic partial differential equations. Elsevier Insights. Elsevier, Amsterdam, 2014.

[14] B. Garrett and R. Gian. Carlo Ordinary differential equations. Fourth edition. John Wiley \& Sons, Inc., New York, 1989.

[15] Y. Giga and H. Sohr. Abstract $L^{p}$ estimates for the Cauchy problem with applications to the Navier-Stokes equations in exterior domains. J. Funct. Anal., 102(1):72-94, 1991.

[16] E. Hausenblas and J. Seidler. Stochastic convolutions driven by martingales: maximal inequalities and exponential integrability. Stoch. Anal. Appl., 26(1):98-119, 2008.

[17] R. He, R. Zhang, and J. Yuan. Noise-induced increase of sensitivity in bacterial chemotaxis. Biophysical Journal, 111:430437, 2016.

[18] T. Hillen and K. J. Painter. A user's guide to PDE models for chemotaxis. J. Math. Biol., 58, 2009. 
[19] D. Horstmann. From 1970 until present: the Keller-Segel model in chemotaxis and its consequences. I. Jahresber. Deutsch. Math.-Verein., 105:103-165, 2003.

[20] D. Horstmann. From 1970 until present: the Keller-Segel model in chemotaxis and its consequences. II. Jahresber. Deutsch. Math.-Verein., 106:51-69, 2004.

[21] N.G. Van Kampen. Stochastic differential equations Physics Reports, 24:171-228, 1976.

[22] E. Keller and L. Segel. Initiation of slime mold aggregation viewed as an instability. J Theor Biol., 26:399-415, 1970.

[23] P. Kotelenez, Comparison methods for a class of function valued stochastic partial differential equations. Probab. Theory Relat. Fields, 93:1-19, 1992.

[24] W. Moon and J.S. Wettlaufer. On the interpretation of Stratonovich calculus New Journal of Physics, 16:055017, 2014.

[25] K. R. Parthasarathy. Probability measures on metric spaces. AMS Chelsea Publishing, Providence, RI, 2005. Reprint of the 1967 original.

[26] C.-S. Patlak. Random walk with persistence and external bias. Bull. Math. Biophys., 15:311-338, 1953.

[27] P. Patniak. Noise in bacterial chemotaxis: Sources, analysis, and control. BioScience, 62:1030-1038, 2012.

[28] B. Perthame. PDE models for chemotactic movements: parabolic, hyperbolic and kinetic. Appl. Math., 49(6):539-564, 2004.

[29] T. Runst and W. Sickel. Sobolev spaces of fractional order, Nemytskij operators and nonlinear partial differential equations. Berlin: de Gruyter, 1996.

[30] A. Stevens. The derivation of chemotaxis equations as limit dynamics of moderately interacting stochastic many-particle systems. SIAM J. Appl. Math., 61:183-212, 2000.

[31] R. L. Stratonovich. Topics in the theory of random noise. Vol. I: General theory of random processes. Nonlinear transformations of signals and noise. Vol. II: Peaks of random functions dnd the effect of noise on relays. Nonlinear self- excited. New York-London: Gordon and Breach Science Publishers. I: XI, 292 p. II: XIV, 329 p. (1967)., 1967.

[32] G. Tessitore and J. Zabczyk. Strict positivity for stochastic heat equations. Stochastic Process. Appl., $77(1): 83-98,1998$.

[33] H. Triebel. Interpolation theory, function spaces, differential operators. 2nd rev. a. enl. ed., 1995.

[34] J.M.A.M. van Neerven, M.C. Veraar, and L. Weis. Stochastic evolution equations in UMD Banach spaces. J. Funct. Anal., 255(4):940993, 2008.

[35] E. Wong and M. Zakai. On the convergence of ordinary integrals to stochastic integrals. Ann. Math. Stat., $36: 1560-1564,1965$.

[36] T. Zhang J. Zhai. 2d Stochastic Chemotaxis-Navier-Stokes System, 2017. J. Math. Pures Appl., 138:307355, 2020.

Department of Mathematics, Montanuniversitaet Leoben, Austria.

School of Mathematics and Statistics, The University of New South Wales, Sydney 2052, AusTRALIA.

School of Mathematics and Statistics, The University of New South Wales, Sydney 2052, AusTRALIA. 